Illinois State University

ISU ReD: Research and eData

Theses and Dissertations

2-7-2019

\title{
Teacher Perceptions of Emergent Bilingual Students and Connection to Pedagogical Resources and Strategy Use
}

Sarah A. Rozny

Illinois State University, srozny@galesburg205.org

Follow this and additional works at: https://ir.library.illinoisstate.edu/etd

Part of the Education Commons

\section{Recommended Citation}

Rozny, Sarah A., "Teacher Perceptions of Emergent Bilingual Students and Connection to Pedagogical Resources and Strategy Use" (2019). Theses and Dissertations. 1038.

https://ir.library.illinoisstate.edu/etd/1038

This Dissertation is brought to you for free and open access by ISU ReD: Research and eData. It has been accepted for inclusion in Theses and Dissertations by an authorized administrator of ISU ReD: Research and eData. For more information, please contact ISUReD@ilstu.edu. 


\section{TEACHER PERCEPTIONS OF EMERGENT BILINGUAL STUDENTS AND CONNECTION TO PEDAGOGICAL RESOURCES AND STRATEGY USE}

\section{SARAH A. ROZNY}

\section{Pages}

This research considers what informs a teacher's dispositions towards his/her emergent bilingual students and in what way these dispositions influence the resources the teacher calls upon and strategies that he/she employs to teach these students. The findings for this study indicate that more positive dispositions towards their emergent bilingual students is connected to exposure to a foreign culture, attempts to learn a foreign language, experiencing teaching emergent bilingual students, and the way the teacher views his or her role as a teacher. More positive teacher dispositions were also connected to a greater use of Just Good Teaching and Linguistically Responsive Teaching strategies. Teachers drew most from personal resources. Teaching resources and strategies often overlapped and influenced one another. Findings led to the creation of a new model for considering teacher dispositions towards emergent bilingual students, which is also presented. This research uses activity theory and positioning theory as the theoretical framework for this exploratory mixed methods study. Qualitative research in this study utilized case study with a strong reliance on grounded theory. For the quantitative phase of data collection, survey data was analyzed using descriptive statistics, T-tests, ANOVA, and a linear regression.

KEYWORDS: emergent bilingual; just good teaching; linguistically responsive teaching; resources; teacher dispositions; teaching strategies 
School of Teaching and Learning

ILLINOIS STATE UNIVERSITY 
Copyright 2019 Sarah A. Rozny 
TEACHER PERCEPTIONS OF EMERGENT BILINGUAL STUDENTS AND CONNECTION

TO PEDAGOGICAL RESOURCES AND STRATEGY USE

SARAH A. ROZNY

COMMITTEE MEMBERS:

Sandra Osorio, Chair

Steven B. Mertens

Kyle Miller 


\section{ACKNOWLEDGMENTS}

Behind every doctoral degree, I believe there must be a team of people keeping the researcher motivated and making progress. I am so grateful for the team that I have in my life. You have made this research better in allowing me to consider my work in ways I had not thought of before and you have made it possible by keeping me motivated and on track!

First, I would like to thank the school district and teachers for welcoming me into their classrooms. Teachers opened up to me about their careers, teaching practices, and their experiences in and out of the classroom. I am grateful to teachers who participated in the survey, as well and to the district who allowed this research to occur.

I am extremely grateful to my dissertation committee members. When I asked Dr. Sandra Osorio, Dr. Steven Mertens, and Dr. Kyle Miller to serve on my dissertation committee, I had strong hopes that each would accept. I am so lucky they did! Not only has their feedback been invaluable in moving this research along and allowing me to consider my proposal and results in different ways, they have all be incredibly supportive. I have felt from the start that these committee members believed in me and that has helped my motivation as I pursued this research.

I would also like to thank Sarah Urbanc and Dr. Donna McCaw. Sarah helped talk through my research with me. Our weekly emails and regular meetings have kept me working hard. There were times when the only motivation I had came from the fact that I knew I would need to be accountable to someone via email at the end of the week! Dr. McCaw, your continuous inquiry into my progress and your insight into managing work and dissertationwriting, kept me going!

Finally, I would not have attempted to start this program, nor would I have been able to complete it without my family. My mom, dad, and sister always encouraged me to learn. School 
was never about grades or job opportunities in my family. Rather, my parents stressed learning for the joy of it and I am a lifelong learner because of this message. Perhaps my greatest supporter in this work is my husband. You encouraged me to continue when I was exhausted and took care of our children when I needed to work long days. You did this in a way that let me know that things would be fine at home without me and that I was setting a good example to my children because of the work I was doing. I love you, Matt.

S.A.R. 


\section{CONTENTS}

Page

ACKNOWLEDGMENTS

TABLES $\quad$ ix

FIGURES

CHAPTER I: STATEMENT OF THE PROBLEM 1

$\begin{array}{ll}\text { Research Questions } & 2\end{array}$

Purpose and Significance of the Study 2

Situating the Study in Current Research $\quad 3$

Practical Implications of the Study $\quad 4$

$\begin{array}{ll}\text { Terms } & 5\end{array}$

Emergent Bilingual

$\begin{array}{ll}\text { Resources } & 6\end{array}$

Disposition $\quad 8$

Deficit Ideology 99

Just Good Teaching $\quad 9$

Linguistically Responsive Teaching $\quad 9$

$\begin{array}{ll}\text { Culturally Responsive Teaching } & 10\end{array}$

CHAPTER II: LITERATURE REVIEW

$\begin{array}{ll}\text { Theoretical Framework } & 11\end{array}$

$\begin{array}{ll}\text { Positioning Theory } & 11\end{array}$

$\begin{array}{ll}\text { Activity Theory } & 13\end{array}$

Review of Literature: Teacher Dispositions 14 
Strategies to Interrupt Deficit Ideology and Factors Correlated with Positive

Dispositions

Review of the Literature: Resources

Review of the Literature: Teaching Strategies

Instruction

Assessment

Culture

Summary and Implications of Literature Review

Research Questions

Research Design

$\begin{array}{ll}\text { Research Approach } & 64\end{array}$

$\begin{array}{ll}\text { Participants } & 65\end{array}$

Qualitative Research Participant Recruitment 65

Quantitative Research Participants $\quad 69$

$\begin{array}{ll}\text { Instruments } & 70\end{array}$

$\begin{array}{ll}\text { Qualitative Data Collection Instruments } & 70\end{array}$

$\begin{array}{ll}\text { Quantitative Data Collection Instruments } & 73\end{array}$

$\begin{array}{ll}\text { Mixed Methods Approach } & 81\end{array}$

$\begin{array}{ll}\text { Research Context and Study Sample } & 83\end{array}$

Unit of Analysis: School District Context 83 
$\begin{array}{ll}\text { Procedure } & 86\end{array}$

Qualitative Data Collection Procedure 86

$\begin{array}{ll}\text { Quantitative Data Collection Procedure } & 88\end{array}$

Combining Qualitative and Quantitative Data 89

$\begin{array}{ll}\text { Data Analysis } & 90\end{array}$

$\begin{array}{ll}\text { Qualitative Data Analysis } & 90\end{array}$

$\begin{array}{ll}\text { Quantitative Data Analysis } & 97\end{array}$

$\begin{array}{ll}\text { Limitations } & 100\end{array}$

$\begin{array}{ll}\text { Positionality } & 100\end{array}$

$\begin{array}{ll}\text { Other Limitations } & 104\end{array}$

Summary: Practical Implications of the Study 105

$\begin{array}{ll}\text { CHAPTER IV: FINDINGS AND RESULTS } & 107\end{array}$

$\begin{array}{ll}\text { Participant Review } & 107\end{array}$

$\begin{array}{ll}\text { Qualitative Teacher Dispositions } & 108\end{array}$

$\begin{array}{ll}\text { Positive Disposition } & 109\end{array}$

Neutral/Positive Disposition 111

Neutral/Negative Disposition 113

$\begin{array}{ll}\text { Deficit/Complex } & 116\end{array}$

Disposition Continuum or a Multifaceted Construct $\quad 120$

Qualitative Findings for Research Question One: What Influences the Dispositions that

Teachers Hold Toward their Emergent Bilingual Students?

Personal Experiences with Bilingual Individuals who immigrated to the United

States in their Childhoods 
Outlook on Teaching and Students in General

International Experience

125

Experience Teaching Emergent Bilingual Students

Quantitative Results for Research Question One: What Influences the Dispositions that

Teachers Hold Toward their Emergent Bilingual Students?

Sub-Question One: Bilingual Status

Sub-Question Two: Travel

Sub-Question Three: Duration of Travel

Sub-Question Four: Length of Time Teaching

Sub-Question Five: Teaching Time Within District

Sub-Question Six: Training for Teaching Emergent Bilingual Students

Sub-Question Seven: Experience with Emergent Bilingual Students

Qualitative Findings for Research Question Two: What Resources and Strategies Do

Teachers Draw Upon in Working with their Emergent Bilingual Students?

Resources

Strategies

Combination Resources and Strategies

Quantitative Results for Research Question Two: What Resources and Strategies Do Teachers Draw Upon in Working with their Emergent Bilingual Students?

Qualitative Findings for Research Question Three: How Does a Teacher's Disposition

Inform the Resources and Strategies They Draw Upon in Working with Emergent

Bilingual Students?

Dispositions Affect Use of Resources and Strategies 
What May be More Influential on Resource and Strategy use than Teacher

Disposition?

Quantitative Results for Research Question Three: How Does a Teacher's Disposition

Inform the Resources and Strategies They Draw Upon in Working with Emergent

Bilingual Students?

Qualitative Findings for Research Question Four: What is the Interplay Between the

Teaching Strategies and Resources that Teachers Use in Working with Emergent

Bilingual Students?

JGT and LRT Strategies and their Connection to Resources

Resources as Strategies/Strategies as Resources

Resources That Allow for Strategies

Conclusion

CHAPTER V: DISCUSSION

Discussion for Research Question One: What Influences the Dispositions That Teachers Hold Toward Their Emergent Bilingual Students?

Discussion for Research Question Two: What Resources and Strategies Do Teachers

Draw Upon in Working with Their Emergent Bilingual Students?

Discussion for Research Question Three: How Does a Teacher's Disposition Inform the Resources and Strategies They Draw Upon in Working with Emergent Bilingual Students? 
Discussion for Research Question Four: What is the Interplay Between the Teaching Strategies and Resources that Teachers Use in Working with Emergent Bilingual

Students?

General Conclusions and Summaries

A New Model of Teacher Dispositions Through the Lens of Activity Theory

Implications

Implications for Professional Development and Teacher Preparation Programs

Limitations

Recommendations for Future Research

APPENDIX B: LANGUAGE ATTITUDES OF TEACHER SCALE BY BYRNES AND 


\section{TABLES}

Table

Page

$\begin{array}{ll}\text { 1. Qualitative Phase Participants } & 67\end{array}$

2. Study Variables $\quad 74$

3. Data Analysis by Question $\quad 90$

4. Teacher Travel and Disposition 126

$\begin{array}{ll}\text { 5. Teacher and Experience } & 130\end{array}$

6. Language Attitudes of Teachers Scale 134

7. Teacher LATS and Emergent Bilingual Training Type 139

8. Teacher and Resource Persons 144

9. Teacher Resource Use 157

10. Mean Resource Use and Teacher Characteristics 157

11. Teacher Strategy Use 159

12. Mean Strategy Use and Teacher Characteristics 160 


\section{FIGURES}

Figure $\quad$ Page

$\begin{array}{ll}\text { 1. Participants Dispositions on a Continuum } & 121\end{array}$

2. A New Model of Teacher Dispositions 196 


\section{CHAPTER I: STATEMENT OF THE PROBLEM}

Within the United States, the percentage of emergent bilingual children has been on the rise (NCES, 2016). According to statistics gathered during the 2013-14 academic year, U.S. schools enrolled 300,000 more students who spoke a language other than English in the home, as compared to data from the 2003-04 academic year and 100,000 more students than just one year prior (NCES, 2016). The increase in this demographic group means that a greater number of teachers will have emergent bilingual students in their classrooms. Emergent bilingual children who represent cultural and linguistic diversity enroll in schools across the country and are located in urban, suburban, rural, and remote areas (NCES, 2016). The need for teachers who are ready to work with the emergent bilingual students is not an issue only reserved for specific states or urban centers. These facts suggest that, more than ever, teachers are going to need the skills and the disposition necessary to teach the English language, how to navigate American culture, and the general coursework.

Policy makers, school administrators, and teacher educators need to be certain that teachers are prepared to work with students who represent a variety of languages and cultures. Preparation must include a foundational knowledge of the way that students acquire and how literacy is learned, as well as well-researched and effective teaching strategies in bolstering the linguistic resources of emergent bilinguals (Filmore \& Snow, 2002). However, one often overlooked aspect of teacher preparation that is the disposition of teachers toward their emergent bilingual student population.

The literature suggests that teacher dispositions affect student achievement (Jussim \& Harber, 2005; Rosenthal \& Jacobson, 1968). These dispositions are especially dangerous to the most vulnerable student populations, such as those from low-income backgrounds (Sorhagen, 
2013). However, few research studies about the factors that shape the dispositions of teachers toward their students and the connection between this disposition and the resources teachers draw upon or the strategies that they choose to use with their students.

\section{Research Questions}

The research questions for this dissertation focus on the teacher dispositions toward their emergent bilingual students and how those dispositions inform the teaching practices that they employ. More specifically, these questions ask:

1. What influences the dispositions that teachers hold toward their emergent bilingual students?

2. What resources and strategies do teachers draw upon in working with their emergent bilingual students?

3. How does a teacher's disposition inform the resources and strategies they draw upon in working with emergent bilingual students?

4. What is the interplay between the teaching strategies and resources that teachers use in working with emergent bilingual students?

\section{Purpose and Significance of the Study}

For approximately 50 years, research has suggested that teachers' dispositions can influence student growth. Positive expectations of students can result in greater gains in IQ, as they did in the landmark study of self-fulfilling prophecies by Rosenthal and Jacobson (1968). Researchers criticized this work, often referred to as the "Pygmalion Effect," and the results were over exaggerated. More importantly, however, researchers frequently repeated the study, new questions on the topic were explored, and the original findings were often replicated (Jussim \& Harber, 2005). A meta-analysis of these studies found that both positive and negative 
expectations could have an impact on student achievement to varying degrees (Jussim \& Harber, 2005).

Literature in the field of teacher disposition and emergent bilingual students demonstrates the need for positive attitudes toward cultural and linguistic diversity to positively prepare teachers to work with emergent bilingual students. A longitudinal study on the impact of teacher expectations on student performance that included more than 1,000 participants found that lowincome children affected the most by teacher expectations (Sorhagen, 2013). This study suggests that children from low-income families whose first grade teachers' over- or underestimated their academic performance were more susceptible to teacher misperceptions in the areas of math and language than students from higher income families (Sorhagen, 2013).

Researchers explored the factors that influence the dispositions of teachers toward their emergent bilingual students in several studies. Research suggests that teachers who have experience learning another language, or who have lived outside of their home country at some point, have more positive dispositions toward culturally and linguistically diverse students than their peers who have not had this experience (Coady, Harper, \& de Jong, 2011; Jiménez, et al., 2015; Pettit, 2011). Research also suggests that empathy-building professional development for in-service teachers and empathy-building instructional practices for pre-service teachers have been effective in developing and strengthening the positive dispositions toward children who represent diverse languages and cultures (Andrei, Ellerbe \& Cherner, 2015; Franquiz, del Carmen Salazar, \& DeNicolo, 2011; Heineke, 2014).

\section{Situating the Study in Current Research}

The research on educating emergent bilingual students is vast and encompasses four branches of study. These branches include (a) how people acquire or learn a language, (b) the 
relationship between language and power, (c) effective teaching techniques for learning both language and content, and (d) the choices and characteristics of teachers who work with emergent bilingual students. Opportunities for further inquiry in this field could involve greater examination of areas where any of these four branches meet or in applying existing theoretical frameworks to research with emergent bilingual students. My dissertation research involves both the intersection of two branches (teaching strategies and teacher qualities), as well as the application of an existing theoretical framework (activity theory).

\section{Practical Implications of the Study}

Understanding what factors influence the dispositions of teachers toward their emergent bilingual students will help teacher preparation programs design educational experiences for preservice teachers. These programs can use the information found in this study to create experiences for pre-service teachers that will foster positive attitudes toward culturally and linguistically diverse people, thereby preparing them to teach the multicultural classes they will serve in the future. Furthermore, a study of the resources and strategies that teachers use will also help pre-service teaching preparation programs understand the tools available to teachers in the field. The study will identify necessary underutilized professional development which can aide in future professional development plans.

Adding to the body of literature on the experiences and characteristics of teachers who are more likely to have positive dispositions toward emergent bilingual students will help school district administrators in making hiring decisions. Literature in the field of education suggests that students live up to teacher expectations (Jussim \& Harber, 2005; Sorhagen, 2013). Therefore, knowing the qualities of teachers with positive attitudes toward emergent bilinguals 
will help identify the right teacher to foster high academic achievement for schools with a diverse student body.

\section{Terms}

Before a discussion of the research can begin, it is important to clarify the terms that I will use throughout this study. Below is a list of the terms that I will use in this dissertation along with a definition of each one.

\section{Emergent Bilingual}

Students who speak a language other than English in their homes may go by many names. Federal and state documents refer to these students as Limited English Proficient (LEP) students. School programs refer to this group of learners as English Language Learners (ELL) or English Learners (EL) who are learning English as a Second Language (ESL) or English as an Additional Language (EAL). However, the literature increasingly uses the term emergent bilingual, multilingual learners, or culturally and linguistically diverse (CLD) students.

For this dissertation, I will use the term emergent bilingual to refer to all students who speak a language other than English in the home and who the school identifies as needing services in English language acquisition. In Garcia's (2009) article on the preference for the term emergent bilingual, she writes:

Calling these children emergent bilinguals makes reference to a positive characteristicnot one of being limited or being learners, as LEPs and ELLs suggest. The term emergent bilinguals refers to the children's potential in developing their bilingualism; it does not suggest a limitation or a problem in comparison to those who speak English. (p.322)

Therefore, using the term emergent bilingual demonstrates the potential of the students to reach a desirable goal of becoming bilingual. Garcia (2009) goes on to state that this term places children 
on a continuum of learning as opposed to opposite ends of a spectrum of proficient or not proficient.

CLD is also a term that does not define students based on a perceived lack of a desirable characteristic, as is the case for ELL or LEP. However, for this dissertation, I am specifically considering the dispositions of teachers of students who require additional assistance in the development of bilingualism. Because some children may come from home environments that are culturally and linguistically different from that of their teachers or the school in general, yet have transitioned out of the need for ESL services, I believe that the term emergent bilingual more accurately represents the students at the center of this study.

Despite the reasons for referring to students as emergent bilingual, in communication with teacher participants, I will use the term "ELL." Teachers are most familiar with this term. This term will be found on the survey, interview protocol, observation letter, and document analysis letter that teachers will receive.

Finally, it is important to note that many of the students in the target district enter the United States speaking more than one language. While I use the term emergent bilingual in this study, the students who work with the teacher participants in the study may be emergent bilingual or emergent multilingual. However, I use the term emergent bilingual to represent both of these groups.

\section{Resources}

Historically researchers often assumed that resources were directly tied to achievement. That is, where resources in the form of money, books, materials, etc. were plentiful, instruction was strong and students would thrive (Cohen, Raudenbush, \& Ball, 2003). In reviewing relevant literature, though, Cohen et al. (2003) identified that the resources that teachers employed in 
their practice went beyond the conventional materials and encompassed qualities such as "instructional practices and organizational arrangements, and the actions, knowledge, and culture that they entail" for both the student and teacher (p. 122). The authors draw a connection to the resources and practices involved in using these resources in the following manner: "The effects of resources depend on both access and use: students and teachers cannot use resources they don't have, but the resources they do have are not self-acting" (p. 122). The use of resources, then, is a choice that teachers make and therefore a pedagogical decision that may be open to further study.

Cohen et al. (2003) considered the many dimensions of school resources that go beyond materials with which teachers and students work. The authors reframe these resources as belonging to one of three streams: conventional resources, personal resources, and environmental resources. For this dissertation proposal, I will consider these resource streams as they fit into the definition provided by Cohen et al. (2003). That is:

Conventional resources include teachers' formal qualifications, books, facilities, class size, and time. Personal resources include practitioners' will, skill, and knowledge. Environmental and social resources include state guidance for instruction, academic norms, professional leadership, and family support. Each type counts. (p. 127)

After interviewing the teachers, it was apparent that teachers used resources in these three different categories. However, the categories can be further divided based on the reasons that teachers use the resources. Each of these three resource categories can be divided into two more sub-categories. There are resources for instruction (that is, those intended to be used directly with students) and resources for pedagogy, (which are resources that teachers use to educate themselves about best practices). 


\section{Disposition}

In the review of literature examining teacher beliefs regarding emergent bilingual students, Pettit (2011) dissected the terms belief, attitude, and knowledge. Each of these concepts may overlap in some ways with the definition of one another, but also slightly differ. For example, a belief or perception may not necessarily be correct or tested for veracity in the same way that knowledge can. Similarly, an attitude does not relate to a single truth. Rather, it is a judgment on something as being favorable or unfavorable (Pettit, 2011).

For the purpose of this study, I will use the term disposition with the understanding that I am referring to the disposition the teachers have toward their emergent bilingual students. The Merriam-Webster dictionary defines disposition as “(a) prevailing tendency, mood, or inclination (b) temperamental makeup, (c) the tendency of something to act in a certain manner under given circumstances" (disposition, 2017). The use of the word "inclination" in the definition indicates that a disposition toward something is similar to an attitude, because it may be related to an evaluation of an object, person, event, or concept (in this case the emergent bilingual students) as being favorable or unfavorable. However, an attitude toward something may indicate a temporary quality, while a disposition is an attitude that lasts longer. This is evident from the words "prevailing" and "tendency" in the definition.

Most importantly, the term disposition fits the purpose of this study precisely because of the third definition provided above. The study seeks to discover the way "the tendency of something [the teacher] act[s] [behaves or teaches] in a certain manner under given circumstances [when working with emergent bilingual students]" (disposition, 2017). Therefore, while the beliefs, knowledge, and perceptions of the teachers may influence the teacher's disposition, in this study, these factors are secondary to the disposition itself. 


\section{Deficit Ideology}

Deficit thinking is a theory that used to explain lower academic performance found among students from low socio-economic backgrounds and students of color (Valencia, 2010). Deficit thinking manifests as a teacher's belief that these students have "limited intellectual abilities, linguistic shortcomings, lack of motivation to learn, and immoral behavior" (Valencia, 2010, p. 7). Those who follow deficit thinking may blame genetics, culture and class, and the way families socialize one another (Valencia, 2010). There are six characteristics of deficit thinking, which include blaming the victim, oppression, pseudoscience, temporal changes, educability, and heterodoxy (Valencia, 2010).

\section{Just Good Teaching}

Just good teaching (JGT) is a term coined by deJong and Harper (2005) to differentiate between teaching strategies that are useful for all diverse learners and those that are specifically beneficial for linguistically diverse students. deJong and Harper (2005) explained that teacher education programs and professional development aimed at general education teachers tend to simplify supportive teaching practices for emergent bilingual students as "a matter of applying 'just good teaching"” (p. 102). These would be "developed for a diverse group of native English speakers, such as activating prior knowledge, using cooperative learning, process writing, and graphic organizers or hands-on activities" (deJong and Harper, 2005, p. 102). However, these practices do not meet all the linguistic needs that emergent bilingual students bring to the classroom (deJong and Harper, 2005)

\section{Linguistically Responsive Teaching}

Linguistically responsive teaching (LRT) strategies are those that are specifically helpful for linguistically diverse students (Lucas, Villegas, \& Freedson-Gonzalez, 2008). Lucas, et al. 
(2008) described LRT practices as "special language-related knowledge and pedagogical competence that mainstream teachers must have to begin to teach ELLs well” (p. 362). LRT practices are those that meet the needs of emergent bilingual students that the JGT practices do not reach.

\section{Culturally Responsive Teaching}

Culturally responsive teaching (CRT) overlaps to some degree with JGT and LRT. CRT refers to teaching strategies that acknowledges and honors students from all cultural backgrounds, regardless of whether or not they are linguistically diverse. Gay argued for "explicit knowledge about cultural diversity is imperative to meeting the educational needs of ethnically diverse students" (Gay, 2002, p. 106). He stated:

Culturally responsive teaching is defined as using the cultural characteristics, experiences, and perspectives of ethnically diverse students as conduits for teaching them more effectively. It is based on the assumption that when academic knowledge and skills are situated within the lived experiences and frames of reference of students, they are more personally meaningful, have higher interest appeal, and are learned more easily and thoroughly. (Gay, 2002, p. 106)

The main difference between LRT and CRT is that LRT are strategies directed at students who speak a language other than English in the home. CRT strategies, on the other hand, are those that support students who may or may not speak English in the home, but whose home culture may differ from the American culture often reflected in schools. 


\section{CHAPTER II: LITERATURE REVIEW}

This chapter considers the theoretical framework that guides this research. It goes into greater depth on the areas of teacher disposition, teacher resources as discussed by Cohen et al. (2003), and teaching strategies for emergent bilingual students. Finally, the chapter concludes with a summary of the connection between these three research strands and how the literature presents them, if at all.

\section{Theoretical Framework}

The research questions call for a multiphase mixed methods study. For the qualitative phase of this study, I used aspects of grounded theory. The definition of grounded theory is "a form of qualitative research developed by Glaser and Strauss (1967) for the purpose of constructing theory grounded in data" (Corbin \& Strauss, 2015). However, in analyzing the data, I drew strongly from two existing theories to allow for an inductive and deductive analysis of data. My perspective on both the literature and methodology draws from the work on positioning theory (Davies \& Harré, 1990) and activity theory (Lantolf, 2000).

\section{Positioning Theory}

It is suitable that for this study I have settled upon the term disposition in referring to the attitudes and beliefs that teachers hold toward emergent bilingual students because the word position itself has been critical in the manner from which I viewed the theories and discussions within the literature. Borrowed from social psychology, positioning theory describes the interaction of individuals, their form of communication, and their stories. Through the act of positioning, an individual communicates who they are in relation to others (Davies and Harré, 1990). Davies and Harré (1990) described this process as being observable, subjective, and "jointly produced" through the interaction of story lines. Positioning is often unintentional and 
can be the result of the way one individual positions another (interactive), or it can be a process in which someone positions himself or herself (reflexive) (Davies and Harré, 1990).

Davies and Harré (1990) argued that positioning is primarily an act of communication and conversation. However, the influence of positioning goes beyond individual-to-individual interactions. Positioning occurs at multiple interactive levels that, within the school, may result in the categorizing students as kinds (Anderson, 2009). Within the classroom environment, positioning students as kinds can influence the participation of students and the manner by which peers and teachers value their contribution (Anderson, 2009).

In the context of teacher interaction with emergent bilingual students, teachers may not be aware of the manner by which their dispositions influence the way they position their students. For emergent bilingual students who may feel, look, and sound different from their peers, the positioning process may begin early. This is especially true for teachers who have a negative disposition toward students who speak languages other than English in the home (Yoon, 2012).

Therefore, one may argue that all teachers working with an emergent bilingual population examine their biases. In a piece written to call for questioning one's teaching craft, Vinz (1997) played with the term disposition to emphasize that educators must remove themselves from their assumptions; that is, teachers need to "dis-position" in order to reconstruct their understandings. She wrote, "shifting valences of dis-positioning may be helpful in allowing us to see more and differently" (Vinz, 1997, p. 145). In reviewing the literature, I paid particular attention to methods by which teachers could dis-position their assumptions. These strategies offer insight into the types of experiences and professional development that may help to form a teacher's disposition toward emergent bilingual students. 


\section{Activity Theory}

Vygotsky developed the start of activity theory, but it was Leontiev in 1978 who developed it into a complete theory (Lantolf, 2000). According to this theory, needs motivate actions. These needs may be biological or they may be culturally constructed. When need is paired with directed action, the need can be described as a motive. Leontiev argued that there are three levels of activities, which consist of motivation, action, and condition (Lantolf, 2000). Of these three levels, one is only able to observe conditions. Different motivations guide actions. These different motivations can create different events even though they are linked to the same actions (Lantolf, 2000).

Through activity theory, motivations affect the manner by which activities are carried out. One way this is especially relevant to language learners is their motivations for learning a language. If a child is motivated to complete a learning task in order to pass a class or appease a teacher, the manner through which they complete the learning activity will differ from students who are motivated to learn a language for the sake of learning or in order to participate in a society that speaks this language (Lantolf, 2000).

The research questions for this study seek to understand any connection between teacher dispositions and the resources and strategies that teachers employ. Survey data and interviews in this research helped in gleaning teacher dispositions. Because Lantolf (2000) wrote that one is only able to observe activity, or conditions, I employed classroom observations and document analysis to observe teaching resources and strategies. In analyzing the data, I considered the motivations of teachers as they worked with emergent bilingual students and any connections this has toward their use of teaching resources and strategies. 


\section{Review of Literature: Teacher Dispositions}

As stated earlier, there is a difference between attitude, perception, beliefs, knowledge and dispositions. While I choose to use the term dispositions, the literature in this area and the literature that I will presently review encompassed all of these terms. Within this review, there is a strong representation of literature that used the term attitude.

One key theme that emerged from the literature of teacher dispositions involved the deficit-based thinking that persists among teachers (Byrnes, Kiger, \& Manning, 1993; Byrnes, Kiger, \& Manning, 1998; DelliCarpini and Alonso, 2014; Franquiz, del Carmen Salazar, \& DeNicolo, 2011; Hutchinson, 2013; Markos, 2012; Salerno \& Kibler, 2013; Stanoschek Youngs \& Youngs, Jr., 2001). Valencia (1997) defined this construct in the following way:

The deficit thinking model, at its core, is an endogenous theory-positing that the student who fails in school does so because of deficits or deficiencies. Such deficits manifest, it is alleged, in limited intellectual abilities, linguistic shortcomings, lack of motivation to learn, and immoral behavior. (p. 2).

Valencia (1997) goes on to describe six characteristics of deficit thinking. These characteristics include: "1)blaming the victim; 2) oppression; 3) pseudoscience; 4) temporal changes; 5) educability; 6) heterodoxy" (p. 3). Of these, the first and fifth may be easiest to identify in teacher discourse. The following studies found that teacher participants often held negative preexisting dispositions toward their emergent bilingual students. They are, in essence, blaming the students for their own educational challenges, rather than seeing the funds of knowledge their students bring to the classroom and the teachers' own agency over their students' learning. These negative dispositions, therefore, match Valencia's definition of deficit thinking. 
Such deficit-based ideology indicates that teachers often overlook the linguistic and cultural abilities as well as the literacy traditions found within the individuals and families who are learning English. Teacher perceptions of the abilities of their students play a role in student achievement (Jussim \& Harber, 2005; Rosenthal \& Jacobson, 1968; Sorhagen, 2013). The following review of literature will discuss the impact of teacher perceptions on student achievement, the extent of deficit-based ideology in both pre-service and in-service teachers, and conclude with a discussion of strategies that have proven to be effective in mitigating negative perceptions of emergent bilingual abilities.

\section{Teacher Expectations and Student Achievement}

As mentioned in Chapter 1, research suggests that student achievement may depend upon teacher dispositions toward these students. Gains in IQ scores as well as performance on standardized tests are two of several measures used to pinpoint such achievement (Rosenthal \& Jacobson, 1968; Sorhagen, 2013). Using a meta-analysis of studies, Jussim and Harber (2005) identified that teachers' expectations of their students can have either a positive or a negative effect on student achievement. Furthermore, in a longitudinal study of 1000 participants, Sorhagen (2013) found that the expectations of a child's first grade teacher had a lasting impact on standardized tests, including those that students took later in their academic careers.

Furthermore, the study found that low-income children were especially vulnerable to low teacher expectations (Sorhagen, 2013).

\section{Extent of Deficit Ideology}

It is, therefore, important for teachers to hold positive dispositions toward their emergent bilingual students. Yet, the research suggests that deficit ideology persists in both pre-service and in-service teachers around the United States (DelliCarpini and Alonso, 2014; Markos, 2012; 
Salerno \& Kibler, 2013). The prevalence of this deficit-based thinking is present in qualitative (Franquiz et al., 2011; Markos, 2012; Salerno \& Kibler, 2013), quantitative (Byrnes et al., 1993; Byrnes, Kiger, \& Manning, 1998; Stanoschek Youngs \& Youngs, Jr., 2001), and mixed methods (DelliCarpini and Alonso, 2014; Hutchinson, 2013) study designs from states across the country. Both pre-service and in-service teachers demonstrate a deficit-based ideology. In qualitative research coming from the English-only state of Arizona, researchers assigned 72 preservice teachers a guided reflection to describe their initial perceptions of emergent bilingual students. These pre-service teachers were asked, "When you hear the word English language learner, what comes to mind?" (Markos, 2012, p. 39). Markos wrote that her "[pre-service teacher students] understandings of ELLs are often based on deficit notions and narrow conceptions of English learners" (Markos, 2012, p. 45). The research, which took place over three semesters, suggested that pre-service teachers based their assumptions of emergent bilingual students on their personal experiences and these initial assumptions were deficit in nature.

Qualitative research conducted by Salerno and Kibler (2013) examine the perceptions of pre-service teachers at a very different stage in their studies. While the participants from the Markos (2012) study were students who registered as education majors, they had not yet taken many courses in their discipline and had not yet participated in an internship experience. However, Salerno and Kibler (2013) collected data from their participants just following the student teaching experience with the purpose "to understand how PST's perceptions of linguistically diverse students appear before they begin full-time teaching and further understand capacities these PSTs demonstrate as they finish preparation" (Salerno \& Kibler, 2013, p. 7). 
Nevertheless, both Markos (2012) and Salerno and Kibler (2013) identified a prevalence of deficit-based thinking in their pre-service teacher participants.

The research by Salerno and Kibler (2013) had several limitations, which may affect the findings. Data collection was limited to a 15-page report on four students that the participants observed and about whom the pre-service teachers collected sample work and interviewed the teacher. Because research data was limited to the report provided by pre-service teachers, key information, such as prior experience working with emergent bilingual students, was unknown. However, Salerno and Kibler (2013) reported that the pre-service teachers did not mention using families as a resource, did not pay particular attention to the student's first language (L1), and viewed speaking in the L1 as an off-task behavior. Such beliefs by pre-service teachers indicate that there is little understanding of the needs of emergent bilingual students and their families and pre-service teachers may need more training in how languages are learned and acquired (Salerno \& Kibler, 2013).

The deficit ideology is not only a problem to be addressed in the largely White, female, and monolingual teacher base. Franquiz et al. (2011) described the need to combat deficit thinking of all pre-service teachers, even for those who are themselves bilingual or teachers of color. The authors purported that pre-service programs do not provide teachers of color an opportunity to examine their own beliefs and biases, which is problematic because these teachers may likewise hold deficit ideologies, which have been developed and internalized in their own educational experiences (Franquiz et al., 2011). It the constant unchallenged acceptance of "majoritarian tales" that perspectives from diverse cultures are silenced and devalued. The authors wrote: 
Over time these tales become commonplace and are accepted as presumed truth. They position members of certain groups as deficient, with qualities that are less than desirable. Such deficit narratives are internalized and brought to teacher-education programs both by White teachers and teachers of color. (p. 282) Therefore, teacher preparation programs must recognize the extent of deficit ideology and allow all pre-service teachers to examine their prejudices.

Using a qualitative design, Franquiz et al. (2011) provided three portraits of pre-service teachers using purposive sampling to demonstrate the extent of existing deficit ideologies in bilingual teachers of color. Researchers selected the three participants from three distinct areas of the country in three distinct preparation programs located in the Southwest, West, and Midwest (Franquiz et al., 2011). The three portraits demonstrated the need for all teachers to unpack the invisible ideologies passed down to them through majoritarian tales (Franquiz et al., 2011).

The deficit views of emergent bilingual students can also be found in studies with inservice teacher participants. (DelliCarpini \& Alonso, 2014; Byrnes et al., 1993; Byrnes, Kiger, \& Manning, 1998; Stanoschek Youngs \& Youngs, Jr., 2001). In a smaller-scale mixed methods study conducted by DelliCarpini and Alonso (2014) with both pre-service and in-service participants, survey findings demonstrated what the authors term "blame the victim' mentality" (p. 168). They go on to write, "the participants generally felt that academic achievement was solely the responsibility of the student and his or her family, while any failure in that area was attributed to a number of personal factors" (DelliCarpini \& Alonso, 2014, p. 168). However, the authors indicated that by the end of the course, teachers became "agentive" as they discussed their responsibility to their emergent bilingual students rather than shortcomings on the part of their students. 
Deficit ideology has been found in quantitative studies as well as the qualitative studies discussed above. In 1994, Byrnes and Kiger published their Language Attitudes of Teachers Scale (LATS) that provides a number for quantifying the disposition of teachers toward emergent bilingual students. Researchers assigned a summative score to respondents based on their answers to 13 Likert-scale questions. One interprets the LATS score in such a way that a greater scored indicates a less tolerant attitudes toward emergent bilingual students (Byrnes et al., 1993). One hundred and eighty-four teachers at a summer workshop completed the LATS survey. Findings of the survey indicated that teachers demonstrated misconceptions about emergent bilingual students, as well as a need for greater training and support in working with this population of students (Byrnes et al., 1993).

Byrnes, Kiger, and Manning (1998), conducted another quantitative study to examine attitudes and knowledge for working with emergent bilingual students. They received 191 responses to their survey that had questions similar to the LATS instrument Byrnes and Kiger had previously used in 1993. Again, the authors found that teachers lacked formal training on teaching emergent bilingual students (less than 17\%) and that teachers believed myths on second language acquisition (Byrnes, Kiger, \& Manning, 1998). Finally, the authors measured attitudes toward emergent bilingual students by asking how teachers would feel about having an emergent bilingual student in their classroom (Byrnes et al., 1998).

One quantitative study regarding teacher attitudes toward emergent bilingual students found more positive results than the findings of the previous studies that I have discussed. Stanoscheck Youngs and Youngs, Jr. (2001) administered a survey in 1996 with in-service teachers in a community located in the Great Plains (Stanoschek Youngs \& Youngs, Jr., 2001). Researchers analyzed the responses of 143 classroom teachers and considered these responses 
alongside factors that correlated with positive attitudes of teachers (Stanoschek Youngs \& Youngs, Jr., 2001). These independent variables included general education experience, ESL training, contact with diverse cultures, prior contact with ESL students, demographic characteristics, and personality (Stanoschek Youngs \& Youngs, Jr., 2001). The authors of this study did not rely upon the LATS 13-item Likert-scale question. Instead, they asked two questions: (a) If you were told that you could expect two or three ESL students in one of your classes next year, how would you describe your reaction? and (b) How would you describe your overall reaction to working with ESL students in your classroom? (Stanoschek Youngs \& Youngs, Jr., 2001). Findings indicate that the teachers who participated in this study demonstrated either neutral or positive attitudes toward emergent bilingual students (Stanoschek Youngs \& Youngs, Jr., 2001, p. 108). Furthermore, they found that:

Mainstream teachers are more likely to have positive attitudes toward ESL students if they have had a foreign language course or a multicultural education course; work in the humanities, social sciences, or natural/physical sciences versus applied disciplines; have had at least some sort of ESL training; have lived outside the United States or taught outside the United States; have interacted with culturally diverse population of ESL students; and are female. (Stanoschek Youngs \& Youngs, Jr., 2001, p. 116) There will be further opportunity to consider the impact of these factors on teacher attitudes in the present research.

The ubiquity of the deficit model applied toward emergent bilingual students indicates a need for research-based practices that allow teachers to disrupt this pattern. What follows is a discussion of research that suggests which strategies can lead to a more positive teacher dispositions toward emergent bilingual students. 


\section{Strategies to Interrupt Deficit Ideology and Factors Correlated with Positive}

\section{Dispositions}

According to Coady, Harper, and de Jong (2011) there are three "dimensions" for preparing teachers to work effectively with emergent bilingual students. These are: “(a) teacher preparation, background, and experience; (b) teacher knowledge of teaching and learning processes with ELLs; and (c) teachers' knowledge of their ELL students" (p. 225). I considered these three variables when developing the independent variables for this study.

During the subsequent review of the literature, I paid particular attention to strategies that school districts or teacher preparation programs could employ to disrupt deficit thinking of emergent bilingual students on the part of the teacher. This study will further test these strategies as they act as the independent variables during the quantitative strand of my research.

Teacher Training. Research suggests that teachers who feel that they have received appropriate training in working with emergent bilingual students have more positive dispositions regarding this population of students (Coady, Harper, \& de Jong, 2011; DelliCarpini \& Alonso, 2014). Similarly, in a quantitative study involving 85 participants, teachers who reported that they felt they had appropriate training for teaching emergent bilingual students also felt more confident about working with these learners (Coady, Harper, de Jong, 2011).

Likewise, a mixed methods study involving both pre-service and in-service teachers found that while participants initially demonstrated more negative dispositions toward emergent bilingual students, their attitudes toward this population became more positive to a significant degree after participating in a course designed to instruct teachers on practices for infusing content-based instruction with language (DelliCarpini \& Alonso, 2014). While the population of this study was limited ( $n=33$ ) (DelliCarpini \& Alonso, 2014), the finding were supported by 
their qualitative phase of research as well as other research in this field (Coady, Harper, \& de Jong, 2011; DelliCarpini \& Alonso, 2014).

Similarly, teachers who participated in a book club format professional development on the topic of teaching writing to emergent bilingual students demonstrated better understanding of the needs of this population of students (Andrei, Ellerbe, \& Cherner, 2015). The authors described this understanding as a "shift" in thinking. While this study did not focus on the general dispositions of teachers toward their emergent bilingual students, the qualitative study based on grounded theory identified, among other things, that "participants left the book club with a more realistic perception of who ELLs are and what they bring to the classroom," (Andrei, Ellerbe, \& Cherner, 2015, p. 17). These findings suggest a strengths-based approach to educating emergent bilingual students.

Tran (2015) used a mixed method design that incorporated a case study component with five cases to determine factors influencing feelings of efficacy in teaching emergent bilingual students and the instructional practices of teachers who felt they were effective. The study drew participants from "two local school districts in central Texas with five or fewer years of experience" (Tran, 2015, p. 32). Findings suggested that "quality preparation experiences" increased teacher feelings of efficacy as well as created more positive beliefs in the abilities of emergent bilingual students. Tran (2015) writes, “These teachers were aware of their student's linguistic strengths to facilitate and enhance ELL's English academic success. Teachers were conscious in recognizing students' language differences as developmental progress, helping students to produce both social and academic language" (p. 38). This research indicated that teachers who have been explicitly taught strategies for teaching emergent bilingual students may be more likely to possess a positive disposition toward these students. 
Participants at the higher education level report increased confidence in meeting the needs of emergent bilingual students following a targeted voluntary professional development for secondary education professors and faculty across departments (Nguyen, Benken, HakimButt \& Zwiep, 2013). The authors acknowledged that the university where the study took place at an "urban state university with faculty dedicated to diversity," which may skew the results toward more positive discourse regarding the abilities of emergent bilingual students than what might take place in other universities that demonstrate less of a commitment to diversity (Nguyen et al., 2013, p. 313). Nevertheless, the authors wrote, "faculty challenged their own knowledge and beliefs/attitudes about how diverse students learn and develop within secondary contexts" (Nguyen et al., 2013, p. 312). This supports other research suggesting the professional development may have a positive influence on dispositions toward emergent bilingual students.

One mixed methods study examined the impact of a required course for pre-service teachers on working with emergent bilingual students (Hutchinson, 2013). One aspect of the required course involved spending three days observing ESL classrooms. Hutchinson (2013) used the LATS instrument for the quantitative strand of research. She identified that the 25 preservice teacher participants demonstrated greater tolerance for emergent bilingual students following the course (Hutchinson, 2013). I would like to note that it is unclear what aspect of the required course had the greatest impact on increasing tolerance of these pre-service teacher participants (Hutchinson, 2013). Furthermore, for some questions, there was a trend toward more conservative political ideology regarding language (e.g., in the post intervention survey, more students agreed that English is a requirement to being considered American and that more students agreed that business should be conducted only in English) (Hutchinson, 2013). 
Proficiency in languages other than English. Both qualitative and quantitative studies report that teachers who speak a language other than English have more positive dispositions toward emergent bilingual students and have more confidence in their ability to teach them (Coady, Harper, \& de Jong, 2011; Stanoschek Youngs \& Youngs, Jr., 2001). The quantitative study conducted by Stanoschek Youngs \& Youngs, Jr. (2001) found that teachers who had studied a foreign language for at least one year demonstrated significantly more positive dispositions toward emergent bilingual students. The work by Coady et al. (2011) supported this finding. In their quantitative study involving 85 participants in their first five years of teaching, Coady et al. (2011) found that teachers who identified themselves as proficient in a language other than English felt more confidence in their preparation for working with emergent bilingual students. This factor was most strongly related to the domain that the authors named social and cultural dimensions of teaching (Coady et al., 2011). However, as the work by Franquiz et al. (2011) suggested, teachers who themselves are bilingual teachers of color may still possess deficit ideology that needs to be examined.

Experience as a minority. For over a decade, researchers have documented the largely homogenous teaching demographics in the United States (National Collaborative on Diversity in the Teaching Force, 2004; Albert Shanker Institute, 2016). Despite a growing population of diverse students within American schools, the teaching force remains primarily White, monolingual English-speakers, and female (National Collaborative on Diversity in the Teaching Force, 2004; Albert Shanker Institute, 2016). While there have been gains in the hiring of teachers from a diverse background, the pace of increasing this population of the teaching force does not match the increase in students from diverse backgrounds (Albert Shanker Institute, 2016). This diversity gap is exacerbated by the greater level of teacher attrition found in teachers 
of color. Therefore, only a relatively small percentage of teachers in American schools can understand what it means to be a minority student or how it feels to be unable to communicate in his or her first language.

Teachers who have had experiences that put them in a minority position or who have experienced what it feels like to be unable to communicate in their first language, may have greater empathy for the challenges of emergent bilingual students (de Oliveira, 2011). While teacher preparation programs and in-service opportunities have used different means of building teacher empathy for emergent bilingual students, research findings suggest that empathybuilding approaches are effective in developing more positive dispositions for emergent bilingual students (Chang, Anagnostopoulos, \& Omae, 2011; de Oliveira, 2011). For example, in a qualitative research design, de Oliveira conducted a simulation in Brazilian Portuguese with 152 pre-service and in-service teacher participants over four years and found that "for those few moments, [teachers] felt what ELLs may feel on a daily basis in schools" (p. 62). In this manner, the study provided teachers with an opportunity to better understand the challenges emergent bilingual students face.

\section{A longer and more targeted experience is the multicultural service learning (MSL)}

programs. In a quantitative study, researchers Chang, Anagnostopoulos, and Omae (2011) applied a multiple regression analysis to results of 212 surveys from pre-service teachers to find similar positive results from empathy-building strategies. This study recognized that students who participated in MSL and had strong support from their MSL site supervisors were more likely to report awareness of preexisting biases and stereotypes than those who did not. The article defined MSL experiences in the following manner: 
MSL represents a distinct type of field experience in teacher education. While most field experiences, such as student teaching, seek to build pre-service teachers' understanding of their professional roles as future teachers, MSL highlights mutual learning and growth between the pre-service teacher as "service learner" and the diverse students and communities being served. (Chang, Anagnostopoulos, and Omae, 2011, p. 1079)

Therefore, MSL is unique in that participants simultaneously learn from and serve their partner communities. The resulting self-awareness of biases and stereotypes may not be the only effect. Research suggests that teachers who have greater experience with emergent bilingual students also have reported increased positive dispositions toward this population (Markos, 2012). Because MSL experiences provides more exposure to certain populations, participants may be more aware of their biases and better able to dispel prejudiced beliefs about the population with whom they work.

Just as the strong support of MSL site supervisors is an important component to questioning one's biases, unpacking one's own experiences may be effective for addressing the deficit ideology that may still exist with bilingual teachers of color (Franquiz et al., 2011). It is through the act of telling one's own story that pre-service teachers were able to question the unstated and invisible stories that value one culture over another (Franquiz et al., 2011). In this way, pre-service teachers were able to identify and therefore work to change their own deficit ideology toward emergent bilingual students (Franquiz et al., 2011).

The work of Stanoschek Youngs \& Youngs, Jr. (2001) also supports the positive benefits of experiencing foreign cultures. Using a quantitative study, Stanoschek Youngs \& Youngs, Jr. (2001) found that teachers who had lived or taught outside of the United States possessed more positive dispositions toward their emergent bilingual students to a significant degree. While 
teachers who travelled outside of the United States also demonstrated more positive dispositions, the results did not yield a significant level (Stanoschek Youngs \& Youngs, Jr., 2001). However, the authors clarify that one limitation of their study is that they cannot determine if teachers who hold dispositions that are more positive seek out these experiences and teachers with less positive dispositions avoid them or if the experiences themselves influence teacher dispositions on emergent bilingual students (Stanoschek Youngs \& Youngs, Jr., 2001).

Experience working with emergent bilingual students. The positive affect of MSL experiences on teacher dispositions toward emergent bilingual students has been previously discussed. The resulting attitude change may be due to a sense of empathy gained from "walking in someone else's shoes." However, MSL experiences may also lead to a stronger sense of respect than other forms of experiences. In a mixed methods study involving 21 pre-service teacher participants, Conner (2010) found that “the prospective educators' views of urban students... became more positive" following an MSL experience (p.1173). What was unique about Conner's (2010) approach is that she "emphasized to the prospective teachers that the learning and teaching were bidirectional, flowing equally between them and the [high school community partners]" (p. 1172). Conner (2010) also avoided the term "service learning" and preferred to highlight that together the pre-service teacher and high school student were "learning partners" to underscore that the pre-service teachers were to learn from the high school students as much as the high school students learn from the pre-service teachers. I would like to point out that the participants in Conner's (2010) study were examining their personal biases toward an urban youth population and not necessarily emergent bilingual students. However, the study nevertheless examined the process of overcoming assumptions and stereotypes, which is pertinent to the proposed study. 
Markos (2012) depended upon self-refection to examine "prior life experiences as a means to reflect on their present position on issues related to educating ELLs" (Markos, 2012, p. 44). Markos (2012) demonstrated that although pre-service teachers were unable to observe classrooms or work directly with emergent bilingual students, over the period of a semester selfreflection of perceptions demonstrated a change to a more positive disposition toward emergent bilingual students. Markos (2012) wrote:

As a teacher-researcher, I believe that it is my job to offer pre-service teachers the time and space needed to examine their thinking. I cannot change my students' beliefs or attitudes. I can only create opportunities for students to acknowledge what they believe, recognize why they believe what they do, and offer counter-stories to challenge and renew their understandings. Ultimately, a change in attitudes and beliefs is the responsibility of the learner. (p. 55)

While Markos (2012) highlighted the importance of self-reflection, it can be inferred that the change in attitudes demonstrated by her students may not be the result of self-reflection alone, but also on the discussions and "counter-stories" offered by Markos herself.

The instructor's role or leader's role may be a strong influencing factor in changes in attitude. Just as Markos (2012) offered counter-stories for participants, opportunities for dialogue about biases were essential in the work of Heineke (2014). Heineke (2014) used literature-based strategies for opening such dialogue and described a literature circle strategy to begin discussions on the challenges and strengths of emergent bilingual students. Heineke's (2014) work suggests that reading and discussing children's literature written by and about immigrants offers a glimpse inside the culturally and linguistically diverse student's life ("windows"), an opportunity for reflection and connection ("mirrors"), and an avenue for connecting to and impacting current 
teaching practices (“sliding doors"). Heineke’s (2014) study participants were pre-service undergraduate and in-service graduate school students seeking an ESL endorsement on their teaching license, which may indicate a more positive initial disposition toward emergent bilingual students as these individuals are working toward enhancing their knowledge and abilities in working with this population. However, her research suggests that using a structured literature circle format allows for dialogue about current perceptions of emergent bilingual students and their experiences and a greater opportunity for developing empathy and understanding of the needs of this population.

In a similar research vein, the qualitative study of Andrei, Ellerbe, and Cherner (2015) that was discussed earlier followed five teachers in a professional development book club that focused on teaching writing to emergent bilingual students. Just as with the literature circles of Heineke's (2014) study, teacher participants of this study noted that the book club format opened dialogue about practices with ELL students. Andrei, Ellerbe, and Cherner (2015) wrote that the "small group encouraged [the teacher participants] to share their voice for the benefit of developing the understandings of the whole group" (2015, p. 14). Therefore, both fiction and non-fiction literature that centers on emergent bilingual or CLD students and their experiences can be effective in providing exposure to this population of learners, and in this way help to reduce biases.

\section{Review of the Literature: Resources}

In Language Attitudes of Teachers Scale, Byrnes and Kiger (1994) measured the resources that teachers have available to them in working with emergent bilingual students in the original study that tested the LATS instrument (Byrnes et al., 1993). To determine the resources available to teachers of emergent bilingual students they asked, "If you have worked with 
[emergent bilingual] students, how helpful (in terms of advice, resources, and assistance) have the following persons been?" (Byrnes et al., 1993). Available options for survey respondents included various local and regional education personnel as well as college and university instructors. The respondents were able to rate the level of helpfulness with "very helpful," "somewhat helpful," "not helpful," "not contacted," and "not applicable” (Byrnes et al., 1993). While the authors did not look for a relationship between the resources and teacher attitudes toward emergent bilingual students, they did identify that teachers primarily seek the help of other teachers for advice in how to educate emergent bilingual students (Byrnes et al., 1993). The authors concluded that the teacher participants need more district and regional support in their work to reduce feelings of isolation and helplessness (Byrnes et al., 1993).

The work by Byrnes et al. (1993) recognized that teachers need more support by way of resources for working with their emergent bilingual students so that they can meet the unique needs of this population. Byrnes et al. (1993) limited their definition of resources to the people available to provide "advice, resources, and assistance" to the teacher of emergent bilingual students (Byrnes et al., 1993, p. 6). However, Cohen et al. (2003) provided an expanded view of resources available to teachers.

For this study, I will use the definition of resources outlined by Cohen et al. (2003) to support the argument that educational research needs to encompass more than traditional considerations of resources. This perspective has been used to study effective professional development on student achievement (Borko, 2004; Penuel, Fishman, Yamaguchi, \& Gallagher, 2007) and the description of student learning as a series of interactions between student and teacher (Doabler et al., 2013). 
I was able to locate one study that employed the theory of different streams of resources to a dual-language environment. Lucero (2015) used a qualitative approach to address questions regarding teacher resources in academic language instruction. She inquired about the resources that teachers use and what factors have influenced the use of available resources. To complete this work, Lucero (2015) used a case study design and drew upon the theory developed by Cohen et al. (2003).

In her work, Lucero (2015) discovered that the resources that dual language teachers used differed from one another. Secondly, the resource streams that teachers employed were described as both "multiple" and "interactive." As mentioned, the environmental resource streams that teachers pulled from varied depending upon their individual personal resources. Furthermore, Lucero (2015) discovered that for one participant there were tensions within one of the resource streams. This tension was found in the way the teachers viewed and used the district-level and school-level ELL teacher. Specifically, Lucero (2015) discovered that the Spanish-speaking teacher was using the ELL teacher to a limited degree as compared with the other first-grade teachers. The ELL teacher believed that the Spanish-speaking teacher did not need her assistance. She reasoned that there was little the ELL teacher could do to help since the other teacher only spoke to her students in Spanish.

While the work by Lucero (2015) indicates that teachers use their resources differently, more research is needed on why some teachers selected to use particular resources over other resources. There may be a number of factors that lead teachers to depend on one resource over another. The present proposed study will determine if teacher dispositions is one factor that influences the resources that they choose to use. 


\section{Review of the Literature: Teaching Strategies}

Working specifically with emergent bilingual students requires the use of intentional strategies that are specifically beneficial to the students who are developing their oral comprehension, production, and literacy in two languages. In 2005, de Jong and Harper laid out the difference between what they call just good teaching (JGT) and what Lucas, Villegas, \& Freedson-Gonzalez, M. (2008) later referred to as linguistically responsive teaching (LRT). De Jong and Harper (2005) recognized the similarities between quality instructional practices for English speakers and emergent bilingual students. However, they described these practices as "insufficient" for the specific needs of emergent bilingual learners (deJong \& Harper, 2005).

LRT overlaps with culturally responsive teaching (CRT). Therefore, this section of chapter two will discuss both of these concepts. The linguistically responsive teacher recognizes the importance language plays as the medium of instruction, but also identifies that the learner cannot be separated from their culture. The culture of the learner serves as the lens through which the content is learned. Therefore, teachers must specifically consider both culture and language when teaching to emergent bilingual students. CRT describes effective teaching strategies for students from a variety of diverse backgrounds and are not necessarily those that are specific to linguistically diverse students. Therefore, teaching strategies that are described as CRT strategies may also be considered JGT (e.g. activating prior knowledge) and other strategies fit more closely with LRT strategies (e.g. building background knowledge using the L1).

For the purpose of this dissertation, I will focus on the strategies found to be beneficial to emergent bilingual students, as well as to all students (JGT), and the strategies that are beneficial specifically to emergent bilingual students (LRT). I have further divided these two categories into the subgroups of knowledge of the student, reading strategies, writing strategies, and cultural 
awareness. I will begin with a discussion of each subgroup and how each of these manifests as

JGT and LRT. Finally, I will describe exact teaching practices in the classrooms that will apply to each of these terms and that I later use for the purpose of coding data.

\section{Instruction}

Languages should represent an additional component in lesson planning (Reyes \& Kleyn, 2010). While the use of translated materials and an atmosphere that welcomes languages other than English will more likely create an environment conducive to learning, research suggests that content should not be delivered twice in two distinct languages. When this happens, students learn to wait until their language is used and tune out the target language (Reyes \& Kleyn, 2010). Instead, teachers should use a combination of just good teaching and linguistically responsive teaching to teach content.

Just good teaching vs. linguistically responsive teaching. In order to create the list of JGT and LRT strategies, I turned to the literature, some of it stretching as far back as the 1990's (Gladson-Billings, 1995; Wlodkowski \& Ginsberg, 1995) in order to read the full extent of the literature on these teaching practices. In the literature on JGT and LRT, several themes began to emerge repeatedly. I grouped these themes into categories that will be discussed below. These categories include general classroom strategies, teaching for understanding, vocabulary, literacy strategies, and oral language strategies. A comprehensive list of JGT and LRT strategies are found in Appendix A.

The literature from the 1990's focused primarily on instructional techniques that were useful for culturally diverse learners. According to de Jong \& Harper (2013), “Often recommendations for 'diverse learners' are actually recommendations for 'diverse native English-speakers' and assume a level of oral language proficiency in order to participate" (p. 
119). Recommendations for diverse native English-speakers can be very effective with emergent bilingual students as well. I categorized these suggestions as JGT strategies. More recent literature in this area began to focus on the distinct and unique needs of linguistically diverse learners. I categorized the strategies that are of specific importance to those students who need to improve their English proficiency as well as learn the content as LRT. Often there is overlap between the two fields. The LRT strategies often take the JGT strategies just one step further.

Routine. There are strategies that teachers can use in just setting up their basic classroom environment that will help diverse learners. Creating a classroom where students feel comfortable and where they are motivated to learn will be useful for all learners. Classroom routines are useful for all learners. When students know what to expect at different times throughout the day, they are better able to focus on instruction. This includes having rules that are consistent, routines that are predictable, classroom management that does not change, and regular routines (Goldenberg, 2008; Lucas, Villegas, \& Freedson-Gonzalez, 2008). Teachers can improve their effectiveness with emergent bilingual students by supplementing the regular routines and procedures with visual aids. These can include schedules that are simple to interpret as well as other diagrams, lists or charts. Teachers can best put these classroom materials to the use if teachers frequently refer to these items throughout the day (Goldenberg, 2008).

Motivating classrooms. Students will increase their motivation when the work teachers provide them is meaningful to the students as individuals and that the students find challenging (Goldenberg, 2008). Classrooms that encourage active engagement and participation are also essential for student motivation (Goldenberg, 2008).

In the 1990's Wlodkowski and Ginsberg created a framework for motivating classrooms in the context of diverse adult learners. Wlodkowski and Ginsberg continued their work in the 
2000's using the same framework. While this context differs in significant ways from the K-12 setting, the four guiding principles of the framework still apply. According to this framework, there are four principles that teachers need to consider in their lessons to make the work motivating. These principles include establishing inclusion, developing attitude, enhancing meaning, and engendering competence. Wlodkowski and Ginsberg (1995) defined establishing as "creating a learning atmosphere in which students and teachers feel respected by and connected to one another" (Wlodkowski \& Ginsberg, 1995, para. 18; Wlodkowski \& Ginsberg, 2009). Such a learning environment would be useful to all learners. Developing attitude is defined as "creating a favorable disposition toward the learning experience through personal relevance and choice" (Wlodkowski \& Ginsberg, 1995, para. 18; Wlodkowski \& Ginsberg, 2009). Enhancing meaning is the process of "creating challenging, thoughtful, learning experiences that include student perspectives and values" (Wlodkowski \& Ginsberg, 1995, para. 18; Wlodkowski \& Ginsberg, 2009). Finally, engendering competence is a matter of "creating an understanding that students are effective in learning something they value" (Wlodkowski \& Ginsberg, 1995, para. 18; Wlodkowski \& Ginsberg, 2009). As with the first concept, these are all teaching and learning processes that would be helpful for all diverse learners, not just adults, and not just those who are linguistically diverse.

Teachers can further create a motivating environment for culturally and linguistically diverse students, when they are aware of including materials, images, and themes that are appropriate (Rhodes, 2017). The appropriateness of these materials may be those that are sensitive to the beliefs of the family's culture. For example, for some students in the target district, reading a book about pumpkins in October would be more appropriate than reading a book about a jack-o-lantern, as the American Halloween tradition is a subject in which there may 
be misunderstanding, fear, or religious objections (Carroll, 2006). A second way to increase motivation for linguistically diverse students is to allow opportunities for students to compare their culture with American culture (Rhodes, 2017). Doing so builds upon a child's existing knowledge and creates a connection between the student and the content.

Teaching for understanding. Just as there are specific techniques that a teacher can do to create a learning environment conducive to the learning for all diverse learners, there are also techniques that teachers can use to increase student understanding in a lesson. Goldenberg (2008) wrote, "What we know about good instruction and curriculum in general holds true for English learners as well, but when instructing English learners in English, teachers must modify instruction to take into account student's language limitations" (p. 14) and strengths. These techniques can be divided into the categories of goals and objectives, content and assessment, independent practice, scaffolding, cooperative learning, extra-linguistic supports, and general instruction.

Learning objectives. Research suggests that lessons that provide clear goals and objectives for learning provide students with the knowledge of what they need to understand (Goldenberg, 2008). This is true for all students. However, teachers can take this concept a step further for their emergent bilingual students. De Jong \& Harper (2013) wrote that "ELL students at the intermediate or advanced stages superficially appear to be able to participate in class, but often miss out on more challenging academic tasks and their performance suffers. Teachers can mitigate this problem by providing explicit language goals and explicitly modeling challenging academic tasks, such as passive voice when reporting a science experiment or teaching discourse features of cause and/or chronology in a history lesson" (p. 2008). This teaching practice requires teachers to identify the linguistic demands of their content area (Lucas, Villegas, \& Freedson- 
Gonzalez, 2008). By paying attention to these demands, teachers can create objectives for both the content they are teaching and the language students will need to understand and produce for this content area (Goldenberg, 2008). Teachers will need to explicitly discuss and teach these linguistic needs (Lucas, Villegas, \& Freedson-Gonzalez, 2008).

Content and assessment. In discussing the content provided to emergent bilingual students by teachers with a deficit-based ideology, Villegas \& Lucas (2007) wrote: Lacking faith in the students' ability to achieve, these teachers are more likely to have low academic expectations for the students and ultimately treat them in ways that stifle their learning. They are more apt to use drill, practice and rote-learning activities at the expense of more challenging work that demands the use of higher-order thinking skills. They are also less likely to call on students in class, give them sufficient wait time to respond thoughtfully to questions or probe incomplete answers for clarity. (p. 5)

However, all diverse learners benefit from a strong curriculum with rich content (Goldenberg, 2008). Teachers can further support this content by using instruction that has a clear design and an appropriate pace for the students (Goldenberg, 2008). When assessing students, portfolios can be helpful for using multiple forms of student work in determining student knowledge rather than solely relying on paper-and-pencil assessments (Smith, 2001). Again, these strategies are good for all diverse learners. For those students who are learning English along with content, teachers need to expect and allow non-verbal responses, especially when the child is new to English or the American school system (Goldenberg, 2008).

Independent practice. Providing students with practice opportunities allows them to apply what they have learned and to transfer this knowledge (Goldenberg, 2008). Teacher feedback on both correct and incorrect student responses further reinforces this learning 
(Goldenberg, 2008). Students can also benefit from review of previously learned material (Goldenberg, 2008) along with assessment and re-teaching when it is required (Goldenberg, 2008). Mastery learning, or the use of standards-based grading, which involves specific objectives to be mastered is useful identifying what needs to be learned and how students can demonstrate learning (Goldenberg, 2008). Research has also identified that allowing student choice in the way that they practice independently has been associated with positive learning outcomes for emergent bilingual students (Rhodes, 2017). Finally, hands-on learning that is concrete is beneficial for all diverse learners (Lucas, Villegas, \& Freedson-Gonzalez, 2008). Strategies that are useful for all learners are categorized as JGT.

Scaffolding. Scaffolding instruction is a technique that many educators learn in their training to become teachers. This is a concept that was developed from Vygotsky's work. Smith (2001) wrote, "Vygotsky emphasized the vital role of imitation. As learners imitate older siblings, parents, or teachers, by mimicking answers, they learn patterns of behavior, vocabulary, and ideas which exceed their present level of development" (p. 222). Imitation is important for all learners. Teachers can provide opportunities for imitation in scaffolding by providing sentence starters or sentence frames for students. Scaffolding is used to move students "between what they already know about a topic and what they need to learn about it" (Villegas \& Lucas, 2007, p. 2). Teachers can make use of this strategy by allowing opportunities to compare their culture to that of American culture (Rhodes, 2017) and by using reading materials in the L1 to build or support background knowledge of the student (Villegas \& Lucas, 2007).

Cooperative learning. The use of cooperative learning is another strategy that helps support all diverse learners (Smith, 2001). This strategy allows students an opportunity to interact in a manner that increases the knowledge of all students (Goldenberg, 2008). Doing so, 
increases student motivation as students create meaning together (Lucas, Villegas, \& FreedsonGonzalez, 2008) in a structured format with guidance from the teacher (Goldengerg, 2008). Cooperative learning can take the form of peer tutors (Rhodes, 2017), student-led discussions (Rhodes, 2017), or a learning theme that has been negotiated by both the teachers and the students (Smith, 2001). For many students, such cooperative learning provides a motivating system of learning. However, for emergent bilingual students, cooperative learning may be crucial as they can use peer translators as well (Lucas, Villegas, \& Freedson-Gonzalez, 2008).

Extra-linguistic supports. Extra-linguistic supports are teaching tools that visually demonstrates a concept or the relationship between several concepts. Again, all students can benefit from the extra-linguistic supports of visual cues, graphic organizers, hands-on activities (Villegas \& Lucas, 2007), pictures, illustrations, maps, videos, graphic organizers, graphs, timelines, and Venn diagrams (Lucas, Villegas, \& Freedson-Gonzalez, 2008). However, emergent bilingual students gain a better understanding of the content when teachers use realia, physical gestures, and visual cues in their teaching (Goldenberg, 2008). Similarly, building redundancy into instruction that allows teachers to repeat and review key ideas and vocabulary provides more support for emergent bilingual learners (Lucas, Villegas, \& Freedson-Gonzalez, 2008). Using multiple ways of providing expectations and directions are also helpful for emergent bilingual students and may include orally providing directions, writing directions down on a board, or giving a handout to students (Lucas, Villegas, \& Freedson-Gonzalez, 2008).

Relationships. Teachers play an important role in influencing their relationships with students and peer-to-peer relationships. Attention to creating a classroom in which particular relationships flourish can influence student learning. All students will benefit from getting to know the child (Lucas, Villegas, \& Freedson-Gonzalez, 2008; Rhodes, 2017). For emergent 
bilingual students, this may take the extra work of getting to know the child's culture and family outside of school hours (Rhodes, 2017). All students benefit from an environment in which they feel safe and are unafraid of taking risks (Lin, 2015). However, for the emergent bilingual student, this becomes especially important. Krashen's (1982) theory of the affective filter suggests that even when students are at a higher English proficiency level, emotion can prevent success when the student feels embarrassed or anxious (Dulay \& Burt, 1977). Teachers can reduce the student anxiety by creating rules that respect all students, reduce competition, and allow for cooperation (Lucas, Villegas, \& Freedson-Gonzalez, 2008).

Regardless of whether or not students represent culturally diverse native English speakers or cultural and linguistic diversity in the classroom, students will benefit from teachers who will advocate for all students (Villegas \& Lucas, 2007), hold positive perceptions of diversity (Nieto, 1996), and are socioculturally conscious. Villegas and Lucas (2007) "define sociocultural consciousness as the awareness that a person's worldview is not universal but is profoundly influenced by life experiences as mediated by a variety of factors, including race, ethnicity, gender and social class" (p. 31). This sociocultural consciousness and positive perceptions of diversity are essential in combatting stereotypes of students and cultures and for teachers to benefit from the effort they put into learning about their students' lives (Villegas \& Lucas, 2007). About developing a knowledge of the student, Villegas \& Lucas (2007) wrote:

We are not suggesting that teachers learn generic information about specific cultural or social groups. Such thinking leads to stereotypes that do not apply to individual students. Instead, teachers need to know something about their students' family makeup, immigration history, favorite activities, concerns, and strengths. Teachers should also be aware of their students' perceptions of the value of school knowledge, their experiences 
with the different subject matters in their everyday settings, and their prior knowledge of and experience with specific topics in the curriculum. (p. 3).

The knowledge that Villegas and Lucas (2007) discussed in this passage is a knowledge on both the individual level as well as the cultural level. Teachers can develop this knowledge through home visits, discussions with students, classroom observations, and conversations with parents and other community members (Villegas \& Lucas, 2007).

Vocabulary. Teachers must pay particular attention to vocabulary development in their work with diverse learners. All students can benefit from explicit instruction in the definitions and use of new vocabulary (Goldenberg, 2008). Goldenberg (2008) wrote that it is most effective when it has been:

Embedded in meaningful contexts and students are provided with ample opportunities for their repetition and used as opposed to looking up dictionary definitions or presenting words in single sentences. Exposure to and use of words in various contexts (reading and hearing stories, discussions posting target words and writing words and definitions for homework. (p. 17)

Teachers can further support vocabulary instruction as they highlight important information of reading material as well as the vocabulary found in text or created for students on a study guide. (Lucas, Villegas, \& Freedson-Gonzalez, 2008; Villegas \& Lucas, 2007).

Teachers of emergent bilingual students can support this vocabulary instruction by encouraging the use of bilingual dictionaries (Villegas \& Lucas, 2007). Knowing which words should be explicitly taught and defined can be supported with teacher knowledge of basic interpersonal communication skills (BICS), or social language, and cognitive academic language proficiency (CALP), or academic language (Lucas, Villegas, \& Freedson-Gonzalez, 2008). An 
understanding of these two linguistic forms and the demands involved in learning each of these will help teachers as they work with their students in developing both their social needs and their academic needs (Lucas, Villegas, \& Freedson-Gonzalez, 2008).

Literacy. As with the other areas previously discussed, teaching literacy to diverse learners has some commonalities regardless of whether the student is linguistically and culturally diverse or if they are native English speakers. All learners benefit from a combination of interactive and direct teaching. Interactive teaching would allow for student practice in the form of editing or discussing, writing, brainstorming, and working with one another (Goldenberg, 2008). However, linguistically diverse students also need teachers to teach directly skills such as letter-sound associations, spelling vocabulary, or modeling (Goldenberg, 2008). Several authors suggested the benefits of process reading and writing (Smith, 2001; de Jong \& Harper, 2005). Goldenberg (2008) used two reports, the National Literacy Panel study of 2006 (August \& Shanahan, 2006) and the Center for Research on Education, Diversity, and Excellence (CREDE) (Genesee, Lindholm-Leary, Saunders, and Christian, 2006) to synthesize the research on teaching emergent bilingual students. He stated, "Process reading and writing received mixed evidence" (p. 18). The process approach was most successful when teachers used it alongside explicit instruction.

For emergent bilingual students, de Jong \& Harper (2013) wrote, "In the area of literacy development, the 'ESL is just good teaching' perspective takes for granted a strong foundation in oral English, i.e. the sound system, vocabulary, grammar, and discourse structure" (p. 117, de Jong \& Harper, 2013). Therefore, teachers cannot depend upon the same strategies that work for other learners who come to school with different linguistic resources. Knowledge of the child's native language ability and their previous literacy learning will give the teacher a better idea of 
what funds of knowledge they bring to the table so that teachers can take advantage of these existing skills (Lucas, Villegas, \& Freedson-Gonzalez, 2008).

Reading. Within the reading domain specifically, there are a number of strategies that will benefit all learners. These include attention to the development of phonemic awareness, phonics, and supporting comprehension through discussions, student and teacher summarizing and paraphrasing, and additional practice in reading (Goldenberg, 2008). Identifying the vocabulary demands in a text and clarifying the definition of these words is also considered a JGT strategy (Goldenberg, 2008).

For emergent bilingual students, when school resources allow for it, teaching students to read in their first language will increase reading skills in English (Goldenberg, 2008). Teachers can also support students reading skills in both the child's first language and English (Goldenberg, 2008). Reading skills gained in any language will transfer to reading skills in English, though some languages will have stronger transfer than others (Goldenberg, 2008). When known, teachers can explicitly teach similarities between the child's first language and English. These include cognates, spelling, and strategies for reaching comprehension. Explicitly teaching these resources will benefit students who may not immediately identify that their existing linguistic resources can support their knowledge of English (Goldenberg, 2008).

In working with students to sound out or decode words, de Jong and Harper (2013) suggested that students should know the word and the meaning before they are asked to "sound it out" (p. 118). Similarly, teachers should be sure that students could perceive and produce different sounds in English before they ask the student to read differences in similar words. Knowledge of the student and the linguistic features of their home language may help teachers know common sounds that need specific, explicit attention (de Jong \& Harper, 2013). While de 
Jong and Harper (2013) suggested that teachers use phonics and knowledge of the student to teach word decoding, they described the practice of asking emergent bilingual students to sound out nonsense words as "misdirected" as they should know the words they are reading first (de Jong \& Harper, 2013, p. 132). The authors also suggested that a strong or weak reading fluency in emergent bilingual students does not necessarily correlate with comprehension in the same way it might with native English speakers (de Jong \& Harper, 2013).

Goldenberg (2008), who synthesized research on effective strategies for emergent bilingual students, suggested that the best way to aid in student comprehension was not teaching comprehension strategies. Rather, teachers can best support comprehension when teachers simplified the texts the students read (Goldenberg, 2008; Lucas, Villegas, \& Freedson-Gonzalez, 2008). However, this recommendation was provided with a caveat that teachers should not overly rely upon simplified texts, which could stymie student growth (Goldenberg, 2008). Other strategies to support students reading comprehension may include supplementing the text with other readings or extra-linguistic support (Lucas, Villegas, \& Freedson-Gonzalez, 2008).

Access to print materials and engagement in literature are important components to literacy development (Cummins, 2012). Literacy development in the students' home languages is essential for transfer to literacy skills in English (Cummins, 2012). Books in the classroom should be available to students in both the target language and the home language and represent the abilities of students (Reyes \& Kleyn, 2010). Ideally, bilingual books that tell a story first in one language and then switch to the other language are preferable to bilingual books that display two languages simultaneously. In this latter format, students will often select to read the text in their stronger language, leaving the target language left unread (Reyes \& Kleyn, 2010). 
Teachers should encourage students to create connections as they read. Connections can be made to students' personal identities, their world, and other academic content areas. These connections can be encouraged by asking students to record their connections in their preferred language through journaling or with sticky notes and to discuss these connections with a classmate (Brooks \& Karathanos, 2009). Selecting appropriate literature that reflects the backgrounds of immigrant students will offer a greater chance of student connection to literature and connection to school (Torrez, 2014).

There is often a tension between the forms of language that our students encounter in school and the language that they experience in their everyday lives (Clark, 2013). The language presented in students' home communities, whether a foreign language or non-standard English, should be valued. Students must learn to speak in a form of English that will offer opportunities beyond school and their immediate communities "without denigrating or disparaging" their home language (Clark, 2013). Clark (2013) suggested that teachers allow for current linguistic practices and localized patterns while also encouraging students to provide their answers, for example, "in the way a historian would say them," thereby adding standard English dialect to the students' dialectical knowledge (Clark, 2013).

Writing. The principals of JGT suggest that all learners will improve their writing with greater writing practice (Goldenerg, 2008) and with additional support for the process writing methods to walk students through the stages of brainstorming, drafting, revising, editing, and publishing (Lin, 2015). Research suggests that students benefit from explicit instruction in strategies for choosing words, rhetorical conventions, and in the difference between quoting paraphrasing, and plagiarizing (Lin, 2015). Furthermore, teachers should provide many opportunities for students to read as a model of writing (Lin, 2015). 
Teachers are encouraged to keep translation to a minimum. If a lesson objective is to write a report in a given language, it is advisable to have reading and research materials provided in that language. Similarly, if a student must define a word, it should be defined in the same language as the word itself (Reyes \& Kleyn, 2010).

LRT principles follow those of JGT, but add two additional techniques that are uniquely important to emergent bilingual students. Students learning English may also need help adjusting to American thought pattern. As teachers provide a model of good writing for their students, they can explicitly teach American sentence structure, writing organization, and discuss the organization of text that they read together (Lin, 2015). In addition, as students learning English, teachers can encourage or allow students to write a first draft in his or her home language and then translating to English once his or her are ideas are down (Lucas, Villegas, \& FreedsonGonzalez, 2008).

A common way for teachers to get to know their students is to assign opinion-writing pieces. De Jong and Harper (2005) cautioned that writing about a students' opinions may not be the 'neutral' writing assignment they assume it to be. There may be cultural taboos against sharing one's personal opinion or a student may be unfamiliar with the topic with which the teacher asks them to write. For example, in a fifth grade classroom, teachers may require students to include detail by asking them to write directions for making a peanut butter and jelly sandwich. If this food is unfamiliar to the student, this assignment would be difficult, not because they cannot perform the writing task, but because the topic is on a subject that is unfamiliar to the student.

Oral language. Oral language includes the language domains of speaking and listening. I will discuss these two domains together. JGT practices include allowing time for extended social 
interaction with both peers and teachers, which will develop both BICS and CALP (Goldenberg, 2008; Lucas, Villegas, \& Freedson-Gonzalez, 2008). Teachers can design for conversations between peers in which the teacher place a facilitative role rather than merely questioning students (Lucas, Villegas, \& Freedson-Gonzalez, 2008). When such conversations are not possible, teachers should be sure to include questions to students to which the teachers do not already know the answers (Lucas, Villegas, \& Freedson-Gonzalez, 2008).

Furthermore, teachers can be sure to speak slowly and deliberately (Goldenberg, 2008), include pauses for a longer period of time or more frequently to provide an opportunity to process the language (Lucas, Villegas, \& Freesdon-Gonzalez, 2008), choose words and expressions that are not idiomatic (de Jong \& Harper, 2013; Lucas, Villegas, \& FreedsonGonzalez, 2008), and otherwise simplify language (Villegas \& Lucas, 2007). The teacher's instructional speech should be varied depending upon the student's linguistic needs Teachers can alter their vocabulary, rate of speech, linguistic complexity, and expectations as needed (Goldenberg, 2008). Teachers can also support the comprehension of their spoken language with the use of extra-linguistic supports or with outlines for lessons (Lucas, Villegas, \& FreedsonGonzalez, 2008). When necessary, teachers can call upon peers to act as translators (Lucas, Villegas, \& Freedson-Gonzalez, 2008). The use of peer translators can be one method by which teachers encourage students to use their native language within the classroom (Lucas, Villegas, \& Freedson-Gonzalez, 2008). Teachers can also encourage students to speak their native language with their families (Rhodes, 2017). Teachers can support the understanding of what they say as they teach in this manner. Therefore, teachers can provide instruction with comprehensible input but must also offer "opportunities to produce output for meaningful purposes" (Lucas, Villegas, \& Freedson-Gonzalez, 2008, p. 363). In creating these opportunities 
for output, teachers must remember that while the silent period is typical. Teachers should not allow ESL students to be "indefinitely silent" and should offer encouragement in attempts to speak English first for social and academic purposes (Lucas, Villegas, \& Freedson-Gonzalez, 2008).

Authors who have written on the topic of JGT and/or LRT practices acknowledge the overlap between these two areas. For example, de Jong and Harper (2005) stated that "activating prior knowledge, using cooperative learning, process writing, and the use of graphic organizers or hands-on activities" (p. 102) are some of those overlapping strategies beneficial to all students of diverse backgrounds. Other strategies that have been found to be beneficial to diverse Englishspeaking students as well as emergent bilingual students are guided reading, writer's workshop, dialogue journals, focusing attention on content-specific vocabulary, and getting to know students on a personal level (de Jong \& Harper, 2005).

Math. Dominguez (2011)addressed the idea of centricity mentioned earlier. He argued against the traditional unidirectional application of in-school vs. out-of-school experiences. Rather, he suggested that not only should math class content be applicable to students outside of the classroom, but also math class should incorporate what is important to bilingual students; that is, being bilingual and their everyday experiences (Dominguez, 2011).

Research conducted with children at various grade levels, speaking languages such as Spanish, Yoruba, and Vietnamese, as well as English, suggested that children will gravitate toward their home language when trying to solve complex math problems. They will also choose their home language in answering open-ended questions about mathematical reasoning. Furthermore, they will perform better in complex math problems when using their home 
language than in English (Dominguez, 2011). In this way, a child's existing linguistic resources can and should be viewed as an additional tool for learning concepts in mathematics.

The participants in Dominguez's (2011) study also indicated that they are more comfortable with exploring new ideas and methods of solving problems when they use their home language, in this case, Spanish. Student participants also produced better results when the topic of the math problem was familiar to them and part of the students daily experiences (Dominguez, 2011).

The work of Lopez, Torres, and Khisty (2013) also supported the findings from Dominguez (2011). In the afterschool bilingual mathematics program they operated, the authors found that students brought with them a variety of linguistic resources. Mathematical reasoning and mathematical discourse is related to communication in this content area as well as "mathematical values, beliefs, and points of view" (Lopez et al., 2013). Each aspect of mathematical discourse needs to be recognized and validated to provide opportunities for success. Furthermore, the linguistic resources brought by students include their understanding of two languages, which may result in either code-switching and/or hybridization of words. Rather than correcting students, Lopez et al. (2013) recommended allowing students to speak freely and recognizing that language is a mathematical resource, even when it does not fit linguistic conventions.

Zaher (2015) claimed that mathematics classes are often shallow in content and goes onto suggest that high-stakes testing may be one of the causes of this shallow curriculum. Due to the pressure of assessments, the curriculum may be constrained leading teachers to focus on instructing students to compute mathematical concepts, but not fully drawing connections for students to understand the reasoning behind these computational procedures. This is true even in 
classrooms of teachers known for mathematically rich instruction for emergent bilingual students (Zahner, 2015). Reinforcing the meaning behind why mathematical computations work rather than merely teaching procedures sets students up for more complex mathematical reasoning in higher-level math classes.

Jarrett (1999) suggested that science and math are good supporters of English language instruction. She wrote that real-world math and science applications, the use of real-world math and science problems, and the incorporation of group work can be used for discussions as well as an opportunity for students to practice explaining their thinking. This, Jarrett (1999) suggested, should be explicitly taught and modeled for students before teachers expect students to practice. The authors suggested that it can be limiting to students to expect them to use only key vocabulary or stick to the math process that the teacher modeled. In other words, accepting multiple forms of arriving at the same answer need to be considered valid.

\section{Assessment}

Emergent bilingual students take a greater number of assessments each year than their general education peers (Gates \& Lichtenberg, 2005). These assessments take students away from much-needed instructional time. Therefore, it is important that the assessments are useful and guide student learning and teaching practices (Gates \& Lichtenberg, 2005). The school should make every attempt to ensure that assessments are valid and reliable. This may mean that assessments should be adapted or modified to meet the needs and abilities of the students.

When giving tests in English, appropriate accommodations provide emergent bilingual students with a necessary advantage in test-taking (Palmer \& Anissa Wicktor, 2008). Such appropriate accommodations would include simplifying the language used in tests and offering extended test time (Palmer \& Anissa Wicktor, 2008). To be effective, the test accommodation 
should match instructional accommodations and be given in the language of instruction (Palmer \& Anissa Wicktor, 2008).

Lucas, et al. (2010) suggested that teachers should do their best to reduce the anxiety that students may encounter when asked to perform in English. Lucas et al. (2010) wrote, "Scaffolding learning for ELLs requires teachers to consider the relationship between students' linguistic abilities and the tasks through which they are expected to learn" (p. 367). We can extend this concept to include scaffolding linguistic abilities and tasks through which they are expected to perform, or be assessed. Some assessment strategies may include reading directions and tests orally and accepting an oral answer. In doing so, teachers can confirm that they are assessing a child's content knowledge and not their reading abilities.

\section{Culture}

Culture is often trivialized to "heroes and holidays." However, an appropriate definition of culture would also contain family structures, value systems, and the way that age, sex, class, and gender intersect (Reyes \& Kleyn, 2010). Understanding student and family culture is important for school-home connections and engaging students as learners.

Maintaining student centricity in the curriculum allows for the content and the curriculum to pull primarily from the students' home culture. Such a practice allows students to make connections between the content and their own experiences (Reyes \& Kleyn, 2010). The connections that students make in the school environment are directly tied to their "community, loved ones, and personal identity" (Brooks \& Karathanos, 2009).

Learning takes place when learning processes call upon existing schema to interpret and connect to new information. Schema forms from one's personal experiences. Therefore, the schema of different individuals will likely be different as well since each person has their own 
set of experiences from which to create schema. Teachers must access the existing schema of students for learning to occur (Brooks \& Karathanos, 2009). It falls to the teachers to learn about and understand their students in order to present lessons that connect to the schema of the students in their classroom and offer appropriate opportunities for making meaning.

Researcher, author, and former emergent bilingual student, Sonia Nieto (2013) described the "wall" that was created for her between home and school as she discovered that her home language and her home culture were considered "low status" within her school. Although she associated her home language and culture with "family and love and nurturing", the experiences at home were not discussed and therefore not valued in school (p. 11). However, as a teacher in a bilingual school, she recognized the importance of ensuring that language and culture are "cherished and affirmed" (Nieto, 2013, p. 13).

Part of student centricity is creating an environment in which students feel comfortable with themselves. School policy may explicitly or implicitly inform students that their language or culture is not valued (Wang, 2009). Feelings of cultural inferiority due to school language policy may be particularly salient when students are immigrants who speak non-European languages at schools that offer only European foreign language classes in addition to the English-based general school curriculum (Wang, 2009). Implicitly restricting student home language use may be a factor contributing to language loss, which can result in loss of cultural identity and communication with family along with the stories and knowledge that can be passed from one generation to the next (Wang, 2009). Therefore, supporting the home language in other classrooms and free spaces in the schools is a necessary component to a student's sense of belongingness and the development of a strong identity. 
Through the idea of Cummins' common underlying proficiency (CUP) model, it is generally understood that provided with the exposure and motivation to learn both languages, the development of a child's first language will support the development of additional languages (Cummins, 1991). Support for the home language extends to school support for the home culture. De Jong and Harper (2005) stated that:

Exclusion of [student linguistic background and cross-cultural experiences] from classroom discourse fails to identify and draw on the skills and strengths that ELLs bring to school, and teachers may subsequently miss opportunities to systematically develop these resources in their curriculum planning and instruction. (p. 113)

Ludhra and Jones (2008) suggested that teachers need to actively and intentionally plan for the student centricity and engaging students through their experiences to make the curriculum relevant to their needs.

Parent engagement. The previous section focused on the importance of centricity in the curriculum to engage diverse student learners. However, creating a classroom environment that derives from a student's home experiences can be a challenge when the curriculum is "culturally and linguistically desensitized" (Torrez, 2014, p. 39). The desensitized curriculum creates an argument that schools and teachers must do the legwork of learning about the experiences of their immigrant families. Hiring staff from the immigrant community allows for a greater schoolhome connection and better insight into the values of the immigrant families that the school serves (Torrez, 2014).

A literature review conducted by Simpson Baird (2015) analyzed the ways in which emergent bilingual families become involved in schools. She found that forms of emergent bilingual family engagement exists on a continuum based around relationships with the 
relationship between families and schools on one end, between parents and children in the middle and among families on the opposing end of the continuum. Relationships between families and schools appear to reflect the "greatest hits" of parent involvement, are schoolcentered, and reflect White, middle class norms (p. $154-155)$. These three forms of parent engagement will be discussed below.

Relationships between families and schools. As Torrez (2014) put it, "a positive school experience is stifled when separation between home and school exists" (p. 39). Parents can support the teaching environment by getting involved in ways that go beyond volunteering at the school and the parent organization. It is therefore imperative that language is not a barrier to parent involvement and that schools welcome parent contributions. Schools can achieve this collaborative parent-school collaborative through parent outreach, parent communication, and parent letters home as well as inviting parents into the classroom on a regular basis (Nieto, 2013).

Increasing the literacy connection between parents and children in the home language improves children's literacy skills in English (Caesar \& Nelson, 2014). Schools can capitalize on this connection by including parents and extended families in literacy assignments. The increase in language proficiency in the home language likely leads to improved proficiency in English (Caesar \& Nelson, 2014).

The concept of CUP was discussed earlier. Coupled with the knowledge that family literacy activities are known to promote literacy development in children, family literacy programs for bilingual or emerging bilingual families that teach parents to engage in literacy activities with children can be successful (Wessels, 2014). This may be particularly true when they utilize a strengths-based approach that seeks to learn from the current practices of parents 
and families (Wessels, 2014). This type of family literacy involvement may also prevent home language loss (Wessels, 2014). However, de Jong and Harper (2005) suggested that there may be different literacy experiences and expectations among various student home cultures. Teacher assumptions regarding the connection between literacy and success in life may not be shared among families of various cultures who do not have a history of literacy and would need to be made explicit for parents of these families.

Relationships between parents and children. The methods of parent engagement described in the previous section primarily reflect what schools can do to encourage families to participate in schools according to White, middle class, American values. However, these activities may be uncomfortable for culturally and linguistically diverse families (Simpson Baird, 2015). Instead, schools can recognize that emergent bilingual families may participate in other forms of engagement with the school through their interactions with their children. For example, emergent bilingual families may prioritize education, modeling and teaching children to respect their teachers, and support schools by disciplining children at home (Simpson Baird, 2015). However, these actions may not be as recognized and valued in the school as they differ from the traditional "greatest hits" of parent involvement. A study conducted by Lim (2012) found that “Asian families' active involvement at home did not receive much recognition from the school, even as these families' strong academic focus was taken for granted due to the parents' high overall expectations" (p. 98). Instead, the immigrant families in the Lim (2012) study were considered "non-participating" (p. 99) because of their limited involvement in school-sponsored involvement opportunities, such as the PTA.

Relationships among families. On the opposite end of the parent involvement continuum from relationships between families and schools is the category of relationships that families 
have with other families who share a common language and culture (Simpson Baird, 2015). This category of research accounted for the fewest type of article identified in the Simpson Baird (2015) literature review and also the most recent research, indicating that it may be a growing trend. This aspect of parent engagement may include the organization of parent groups that can play a transformative role in school improvement. For example, Lim (2012) describes the purpose of a Korean immigrant parent group in the following manner: "In sub, the Korean parent meeting helped to fortify Korean families against the disadvantages they might encounter as a minority immigrant group" (p. 103). These "disadvantages" were ameliorated by aiding one another in understanding the "structural barriers" to school involvement, such as "the language barrier, time constraints, and lack of knowledge about school culture" (Lim, 2012, p. 104). Essentially, helping one another understand the cultural differences between their home country and the United States allows other families to navigate these structural barriers in order to help one another be more successful in school.

All three forms of parent engagement is important to a school. Simpson Baird (2015) writes, "While the greatest hits emphasizes specific, observable practices that parents might be seen engaging in either at home or at school, the studies included in this review reveal that EL parent involvement might be less obvious" (p. 168). Therefore, it is imperative that schools not only encourage family involvement by emergent bilingual parents and provide support to allow for this to take place in traditional formats, but they must also recognize when parents are engaged in other methods as well.

\section{Summary and Implications of Literature Review}

The literature suggests that teachers who have low expectations of their students may affect the success and achievement of these students. This is particularly true for emergent 
bilinguals and other vulnerable populations. However, while it is documented that deficit ideologies are prevalent among both pre-service and in-service teachers toward their emergent bilingual students and there are studies that demonstrate more positive dispositions or at least stronger feelings of teacher efficacy following professional development, few studies suggests what factors influence the dispositions that teachers hold (Stanoschek Youngs \& Youngs, Jr., 2001). The existing literature in this area is limited and dated. It could benefit from further research.

Little to no research exists that examines the connection between the dispositions that teachers hold and the resources that they call upon in their practice. Likewise, little to no research exists that connect their dispositions to the teaching strategies that they choose to employ with their emergent bilingual students. The present proposed study seeks to understand this connection and fill this gap in the literature. 


\section{CHAPTER III: METHODOLOGY}

The previous two chapters discussed the importance of studying the proposed research questions and how these questions fit in the wider literature base. In this chapter, I will provide an in-depth description of the process I used to answer the research questions. In order to accomplish this task, I will begin with a review of the research questions in this study.

\section{Research Questions}

The research questions at the center of this study are below. Because the first three questions were addressed using both qualitative and quantitative means, a null hypothesis has been provided for these questions, the first of which has seven sub-questions, each with its own null hypothesis. I addressed the last research question using only a qualitative research design and therefore I have not provided a null hypothesis for this question. For the purpose of brevity, the use of the term "disposition" in the hypotheses refers to the disposition of the teacher participants toward emergent bilingual students, as measured by the Language Attitude of Teachers Scale (LATS) (Byrnes \& Kiger, 1994).

1. What factors influence the dispositions that teachers hold toward their emergent bilingual students?

a. Sub-question One: Do the dispositions of teachers who speak a language other than English differ from the dispositions of teachers who do not speak a language other than English?

$\mathrm{H}_{0}$ : There is no relationship between dispositions of teachers and whether or not the teachers speak a language other than English. 
$\mathrm{H}_{1}$ : The dispositions of teachers who speak a language other than English will not be equal to the disposition of teachers who do not speak a language other than English.

b. Sub-question Two: Do the dispositions of teachers who have visited a country that speaks a language other than English differ from the dispositions of teachers who have not visited a country that speaks a language other than English?

$\mathrm{H}_{0}$ : There is no relationship between dispositions of teachers and whether or not the teachers have visited a country that speaks a language other than English. $\mathrm{H}_{2}$ : The disposition of teachers who have visited a country that speaks a language other than English will not be equal to the disposition of teachers who have not visited a country that speaks a language other than English.

c. Sub-question Three: Do the dispositions of teachers who have spent a longer amount of time in a country that speaks a language other than English differ from the dispositions of teachers who have visited a country that speaks a language other than English for a shorter amount of time?

$\mathrm{H}_{0}$ : There is no relationship between dispositions of teachers and the length of time a teacher has spent in a country that does not speak English.

$\mathrm{H}_{3}$ : The disposition of teachers who have spent a longer amount of time in a country that speaks a language other than English will not be equal to the disposition of teachers who have visited a country that speaks a language other than English for a shorter amount of time. 
d. Sub-question Four: Do the dispositions of teachers who have spent a longer amount of time teaching differ from the dispositions of teachers who have been teaching for a shorter amount of time?

$\mathrm{H}_{0}$ : There is no relationship between dispositions of teachers and the length of time a teacher has spent teaching in general.

$\mathrm{H}_{4}$ : The disposition of teachers who have spent a longer amount of time teaching will not be equal to the disposition of teachers who have been teaching for a shorter amount of time.

e. Sub-question Five: Do the dispositions of teachers who have spent a longer amount of time teaching within the school district at the center of this study differ from the dispositions of teachers who have been teaching for a shorter amount of time within the study school district?

$\mathrm{H}_{0}$ : There is no relationship between dispositions of teachers and the length of time a teacher has spent teaching in the study school district.

$\mathrm{H}_{5}$ : The disposition of teachers who have spent a longer amount of time teaching within the study school district will not be equal to the disposition of teachers who have been teaching for a shorter amount of time within the study school district.

f. Sub-question Six: Do the dispositions of teachers who have had more training in teaching emergent bilingual students differ from the disposition of teachers who have had less training in teaching emergent bilingual students?

$\mathrm{H}_{0}$ : There is no relationship between dispositions of teachers and the amount of training a teacher has had in teaching emergent bilingual students. 
$\mathrm{H}_{6}$ : The disposition of teachers who have had more training in teaching emergent bilingual students will not be equal to the disposition of teachers who have had less training in teaching emergent bilingual students.

g. Sub-question Seven: Do the dispositions of teachers who have had more experience teaching emergent bilingual students differ from the disposition of teachers who have had less experience teaching emergent bilingual students?

$\mathrm{H}_{0}$ : There is no relationship between dispositions of teachers toward emergent bilingual students and the amount of experience a teacher has had in teaching emergent bilingual students.

$\mathrm{H}_{7}$ : The mean disposition of teachers who have more experience teaching emergent bilingual students will not be equal to the disposition of teachers who have had less experience teaching emergent bilingual students.

2. What resources and strategies do teachers draw upon specifically for working with their emergent bilingual students?

$\mathrm{H}_{0}$ : There is no relationship between the use of additional resources for teaching and the number of emergent bilingual students enrolled in the teacher's classroom.

$\mathrm{H}_{1}$ : The mean use of teaching resources used by teachers with emergent bilingual students enrolled in the classroom will differ from the mean use of teaching resources used by teachers with fewer emergent bilingual students enrolled in the classroom. 
$\mathrm{H}_{0}$ : There is no relationship between the use of "Just Good Teaching" (JGT) and "Linguistically Responsive Teaching" (LRT) strategies for teaching and the number of emergent bilingual students enrolled in the teacher's classroom.

$\mathrm{H}_{2}$ : The mean use of JGT and LRT strategies used by teachers with emergent bilingual students enrolled in the classroom will differ from the mean use of JGT and LRT strategies used by teachers with fewer emergent bilingual students enrolled in the classroom.

3. How does a teacher's disposition inform the resources and strategies they draw upon in working with emergent bilingual students?

H0: There is no relationship between the dispositions of teachers and the number of resources specifically used for teaching emergent bilingual students.

$\mathrm{H}_{1}$ : The mean use of teaching resources used by teachers with more a more positive dispositions toward emergent bilingual will differ from the mean use of teaching resources used by teachers with a more negative disposition toward emergent bilingual students.

H0: There is no relationship between the dispositions of teachers and the number of JGT and LRT strategies specifically used for teaching emergent bilingual students.

$\mathrm{H}_{2}$ : The mean use of JGT and LRT strategies used by teachers with more a more positive dispositions toward emergent bilingual will differ from the mean use of JGT and LRT teaching resources used by teachers with a more negative disposition toward emergent bilingual students.

4. What is the association between the teaching strategies and resources that teachers use in working with emergent bilingual students? 


\section{Research Design}

In the present section, I provide an overview of the methodology for this study as well a discussion on the way that I approach this study. I will go into detail on each part of the methodology in subsequent sections. The questions posed in this study call for a sequential mixed methods study design that uses the exploratory format of qualitative(qual) $\rightarrow$ quantitative(quant). Exploratory sequential design is defined as "a two-phase mixed methods design in which the researcher starts with the collection and analysis of qualitative data, followed by the collection and analysis of quantitative data to test or generalize the initial qualitative findings" (Creswell \& Plano Clark, 2011, p. 411). I addressed the first three research questions by using both a qualitative and quantitative approach. The qualitative work uses a case study design and pulls strongly from grounded theory while the quantitative analysis will use $t$-tests, analysis of variance (ANOVA), descriptive statistics, and linear regression to analyze the data.

Case study design is appropriate when the research questions under study examine an event or phenomenon currently taking place and the research cannot demonstrate control over these events (Yin, 2014). Yin writes, "The more that your questions seek to explain some present circumstance (e.g., 'how' or 'why' some social phenomenon works), the more that case study research will be relevant" $(2014$, p. 4). In this study, the purpose of the research is to determine how teachers form their dispositions toward emergent bilingual students, why teachers decide to draw upon particular resources or strategies when working with emergent bilingual students, and how their disposition plays a role in the selection of resources and strategies. For this reason, case study methodology supports the grounded theory approach toward arriving at answers to the research questions. 


\section{Research Approach}

According to Delgado Bernal (2002), "Epistemology, in general, refers to the nature, status, and production of knowledge (Harding, 1987) and the way one knows and understands the world" (p. 106). The researcher's epistemology is the paradigm the researcher uses to interact and explain their world and therefore the process by which they conduct research and analyzes data. For this reason, a discussion of my personal epistemology is important in order to consider the process I have chosen to analyze my questions and remained important as I proceeded with implementing the research plan.

My epistemology is most closely aligned with critical social theory. In attempting to draw connections to the different schools of thought in critical theory, Kincheloe, McLaren, and Steinberg (2011) describe a list of assumptions that are true for all critical theorists. While the list contains seven bullet points, the first of these points gets to the heart of critical theory. It states that "All thought is fundamentally mediated by power relations that are socially and historically constructed" (p. 164). The authors state that despite the existence of various strands of critical theory, each critical theory agrees that within all societies there are some groups that are afforded more rights and privileges than others (Kincheloe et al., 2002). Furthermore, most research reproduces society's “systems of class, race, and gender oppressions” (p. 164). Critical theory argues that all students are holders and creators of knowledge (Delgado Bernal, 2002). Researchers who subscribe to critical theory conduct research with the intent to improve the world. Critical theory is closely tied to empowerment and transformation (Kincheloe et al., 2011). In this manner, critical theory is set apart from the idea of research as apolitical and neutral (Kincheloe et al., 2011). 
Due to the nature of critical theory, as a researcher, I am aware of the power system within schools in general between the teacher and student. Furthermore, the emergent bilingual students who, while not participants, are central to this study are immigrants and are often, though not always, racially and ethnically different from their teachers, most of whom are White. The power dynamic of this relationship is also acknowledged in the research process.

Simultaneously, I am aware that the power relationships within many school districts involve an often White, male administration leading policy that will be implemented by a primarily White and female teaching staff. I recognize the importance of knowing that the teachers who are the participants of this study are in some ways themselves an oppressed group. They, too, are often unrecognized holders and creators of knowledge. In this regard, I aim to focus on the strengths that teachers bring to their work in their classrooms.

\section{Participants}

\section{Qualitative Research Participant Recruitment}

The target population for this study is elementary school general education teachers who work with emergent bilingual students in the third through fifth grades. I have narrowed down the participating teachers to only those who work with these grade levels for several reasons. First, as a former elementary school teacher myself, I am more familiar with the content and teaching strategies for this age group than I am with middle school or high school. As a researcher, therefore, I am more aware of when a teacher is using strategies specifically for the emergent bilingual students in his or her room than I would be in other grade levels.

Secondly, at the elementary school level, education of younger students is characterized by, among other things, language development. All students, native English-speakers and emergent bilingual students alike are work on foundational skills of reading, writing, speaking, 
and listening (Common Core State Standards Initiative, 2019). By third grade, Common Core State Standards require decoding multisyllabic words and by fourth grade students are expected to be able to read fluently listening (Common Core State Standards Initiative, 2019). Therefore, strategies that teachers use for teaching language and literacy may be suitable for all students making it difficult to glean what teachers are doing intentionally for teaching new vocabulary and meeting other needs of emergent bilingual students.

Furthermore, it would be easier to compare teaching strategies of teachers who are working within first through second grades of one another rather than comparing, for example, the teaching strategies of a first grade teacher with that of a high school teacher. As de Jong and Harper (2013) write,

While elementary students continue to be provided with hands-on activities, manipulatives, visuals, and small group and whole group discussions to help mediate and scaffold their learning (including language learning), secondary level students are expected to be prepared to learn in and through language. (p. 124).

Therefore, limiting the grade range of the participating teachers allowed for teacher dispositions, resources, and strategies data to be considered in light of the elementary school practices of that building and the general expectations for students at that age level.

In this study, I observed any subject area taught by the participating teacher. These include math, science, social studies, reading, and language arts. Each of these subjects provided opportunities for differentiation and targeted language instruction.

I selected teacher participants for the qualitative phase of data collection from one school district. While they all stated they had positive dispositions toward emergent bilingual students, there were differences between their dispositions as well. The teachers employed a variety of 
teaching strategies when working with this population. Because there are only 24 third through fifth grade teachers in the school district who work with emergent bilingual students during the year of the study, I invited all teachers to participate. Ten of the 24 teachers agreed to participate. I gave preferential selection to teachers who I have not worked with in the past and who have the highest number of emergent bilingual students in their classrooms. However, I also worked to find teacher with a diverse range of experience both in the number of years they taught emergent bilingual students and in the number of emergent bilingual students they taught. Teachers were also included if they presented unique experiences that may inform their teaching of emergent bilingual students. Ultimately, I selected the following teachers for the study:

Table 1

Qualitative Phase Participants

\begin{tabular}{ccccc} 
Pseudonym & Years Teaching & \# of EB Students & Other & School \\
\hline Holly & 3 & $40+$ & Husband is & A \\
& 22 & & Immigrant & B \\
Stephanie & 11 & 4 & None & C \\
Jennifer & 39 & 3 & Board Certified & A \\
Susan & 19 & 12 & None & C \\
Melody & 22 & 3 & Multilingual and & D \\
Linda & & & Immigrant \\
\end{tabular}


However, in selecting teacher participants for this study, I kept in mind advice offered by Stake (1995) who emphasized that "Case study research is not sampling research" (p. 4). In other words, it is difficult, or impossible to find cases that will represent a larger group as each case is distinct. Furthermore, Stake (1995) suggested that unique cases may occasionally be more informative about the larger sample. He suggested that while it is important to choose samples that offer variety in the cases, it is of primary concern to select cases that offer the "opportunity to learn" (p. 6).

While the qualitative component of this study used case study design, it also borrowed heavily from a grounded theory approach. According to case study methodology, each case is a unit of study and multiple units of study may be needed to address the research questions (Yin, 2014). Just as with theoretical sampling, researchers using a case study design are warned that "Your choice of the unit of analysis, as with other facets of your research design, can be revisited as a result of discoveries during your data collection" (Yin, 2014, p. 32). Therefore, it is an accepted practice to select cases based on input provided by the gatekeeper and even alter cases as necessary.

Yin (2014) recommends, "bounding the case" in order to help identify cases that answer the research questions (p. 33). The researcher must consider the questions that need to be answered, as well as the allowed period in order to accomplish this task. For the present study, I examined six cases in this multiple case study method that represented a range of dispositions toward their emergent bilingual students. Because I conducted classroom observations and completed the qualitative data collection within a single school year, limiting the number of cases to six made these tasks more feasible. 


\section{Quantitative Research Participants}

The participants for the quantitative phase of data collection consisted of general education teachers within the same school district from which the qualitative phase participants were selected. The district employs teachers for grade levels preschool through grade 12 who teach in one preschool, five elementary schools, two junior high schools, one high school, and one alternative school. According to the Illinois State Board of Education (2017), the teacher population of this school district consist of teachers who are 91.5\% White and $77.8 \%$ female.

I recruited participants for the quantitative phase of data collection by sending an email to a district-wide email list of 297 teachers. I have access to this listserv as a district employee and received permission to use it as a researcher. The survey, which can be found in Appendix B, was sent to all district teaching staff via a district listserv. The survey was mailed to teachers for three consecutive weeks in October 2018. The teachers receiving the survey consisted of 297 teachers who teach in the capacity of classroom teachers, specials teachers (such as art, music and physical education), special education teachers, ESL teachers, speech teachers, outreach workers, and one dean of students. Of these teachers, 55 completed the survey. This is a response rate of $18.5 \%$. This response rate is relatively low compared to similar research studies.

While the outreach worker and dean of students were included in this study, they only account for three staff members out of the entire district. There are two outreach workers and one dean of students. I included this group in the category of "other" so that the identities and practices of these participants would remain anonymous.

According to Creswell and Plano-Clark (2011), ideally, the participants for exploratory design of sequential mixed methods recruit separate participants from the qualitative and quantitative strands of data collection. However, for this study, the teachers from the qualitative 
phase of data collection were included in the quantitative phase as well. These teachers were included because the sample size was limited.

\section{Instruments}

\section{Qualitative Data Collection Instruments}

I collected qualitative data prior to the quantitative data. For this phase of research, I used observations, interviews, and document analysis. I will discuss the instruments used for collecting this data below.

SIOP for observation. Observations were conducted two times during the qualitative data collection period. I recorded field notes for this observation and use the Sheltered Instruction Observation Protocol (SIOP) to help ensure consistency between observations. The protocol is in Appendix C.

The purpose of the observation protocol form was be to ensure that I was recording information relevant to research-based teaching strategies and that I was looking for similar practices amongst all the teachers. The observations helped to answer research questions two through four. That is, the observations provided information pertinent to teacher resources and teacher strategies. Therefore, the observation protocol focused directly on the teaching strategies that teachers may employ with their emergent bilingual students.

The SIOP instrument is a tool that has been developed using what research suggests are effective practices for teaching both content and the English language to emergent bilingual students (Echevarria, Vogt, \& Short, 2017). The authors of the SIOP instrument reviewed research that focused on "best practices for English learners in the areas of ESL, bilingual education, reading, second language acquisition, discourse studies, special education, and classroom management” (Echevarria et al., 2017, p. 317). Two of the authors of the SIOP 
instrument tested combinations of the best practices in classrooms over the course of four years until a model of effective teaching techniques was developed in 2000 (Echevarria et al., 2017). While the authors of the SIOP protocol claim that the instrument has been found to be "a valid and highly reliable measure of sheltered instruction" (Echevarria et al., 2017, p. 318), other research suggests that the claim was made based on a small sample size with a minimal effect size (Krashen, 2013). However, the instrument does provide a research-based tool for determining the use of effective teaching strategies for emergent bilingual students and so is appropriate for the given study.

The instrument consists of 30 items divided into the categories of lesson preparation, building background, comprehensible input, strategies, interaction, practice and application, lesson delivery, and review and assessment. Each of the 30 items are to be observed and then scored on a scale of 0-4, with 0 indicating "not evident" to 4 "highly evident" (Echevarria et al., 2017). There are five items for which "not applicable" is also a choice (Echevarria et al., 2017). In addition to the categories included in the SIOP protocol, I have added three questions regarding the classroom environment and the inclusion of home languages and cultures. These questions will be created in a format similar to those of the SIOP protocol, they will also be assessed on a scale from 0 (not evident) to 4 (highly evident).

While the purpose of the SIOP scale is to quantify the teaching strategies that teachers use, that is not how I use this tool. Because the observations are a part of the qualitative data collection, the quantification of teaching strategies are not necessary for the research. Rather, this instrument is used only for guiding observations. 
As I observed teachers, I also kept an observation log. The SIOP instrument helped to narrow the focus of the observation. This log was then reviewed and coded for patterns and themes and also to identify JGT and LRT teaching strategies.

Document analysis. While I observed classrooms, I asked teachers to provide a copy of their lesson plans as well as any documents that all students and emergent bilingual students specifically use for their work. The documents I viewed include handouts, worksheets, homework, and lesson plans. The document analysis checklist includes 12 items specifically associated to lesson plans. There was also be an opportunity to record additional notes, which may be particularly useful as teachers likely will not record their lesson plans in the same format.

There are three questions related to the handouts, assignments, and assessments. These questions focus more on the accommodations and modifications that may be represented in the materials because these materials are designed specifically for student use. The questions ask if the assignment or assessment have been modified, if modifications are tailored to individual student needs, and if the modifications or accommodations are consistent with modifications or accommodations made during the time of instruction (Appendix D). The document analysis protocol includes fewer items for consideration than the SIOP instrument to allow for the anticipated diversity in classroom handouts and assessments. These questions offered an opportunity for notes to be recorded on the documents under analysis.

Semi-structured interviews. Two semi-structured interviews with case study teacher participants were administered. Semi-structured interviews, or semi-structured life world interviews, are defined as "a planned and flexible interview with the purpose of obtaining descriptions of the life world of the interviewee with respect to interpreting the meaning of the described phenomena" (Brinkmann \& Kvale, 2015, p. 367). A semi-structured interview format 
was selected because this type of interview process provides the structure of defined interview questions with the freedom to clarify responses. Because interviews were to be coded according to specific categories related to resources, I used a semi-structured format so that I may seek clarification of interview answers as they relate to coding categories per the recommendation of Brinkmann and Kvale (2015).

Interview scripts for the two interviews are included in appendices E and F. Interview questions have been designed with the research questions in mind (Brinkmann \& Kvale, 2015). Therefore, for the purpose of this study, the interview scripts have been written with the research questions in the left-hand column and the interview script questions in the right-hand column.

Although the interviews are related to the research questions, each interview primarily focused on specific research questions. The first interview instrument focused on the teacher's disposition and experience. This interview only briefly touched upon their plans for resources and strategies and was composed of 10 questions. The second interview was composed of 13 questions. Additional clarifying questions from the observations and previous interview were also included. This interview reviewed the teacher's feelings toward their experience of working with their emergent bilingual students. In doing so, this interview included two questions that came from a study by Stanoscheck Youngs and Youngs, Jr. (2001) to identify disposition. These were the only two questions that were derived from the literature. The interview questions were not piloted. These questions and this study will be discussed in greater depth in the subsequent section. It also asked teachers to reflect upon their use of resources and teaching strategies.

\section{Quantitative Data Collection Instruments}

Before I describe the instrument that I have used to collect and analyze quantitative data, I will need to mention the independent variables. My proposed study will include several of the 
independent variables used in the study by Stanoscheck Youngs, and Youngs, Jr. (2001). The variables of this study are outlined in Table 2 . Those marked as " 2001 " are the variables that were explored in the Stanoscheck Youngs, and Youngs, Jr. (2001) study and were revisited during this study.

Table 2

Study Variables

\begin{tabular}{|c|c|c|c|c|c|}
\hline Variable & Nominal & Ordinal & Interval & 2001 & $\begin{array}{l}\text { IV/ } \\
\text { DV }\end{array}$ \\
\hline Bilingual & $\mathrm{x}$ & & & $\mathrm{x}$ & IV \\
\hline Travel & $\mathrm{x}$ & & & $\mathrm{X}$ & IV \\
\hline Travel Time & & $\mathrm{x}$ & & $\mathrm{x}$ & IV \\
\hline Teaching Time & & $\mathrm{x}$ & & $\mathrm{X}$ & IV \\
\hline Teaching in District & & $\mathrm{X}$ & & & IV \\
\hline Training with EB & $\mathrm{x}$ & & & $\mathrm{x}$ & IV \\
\hline EB Experience & & $\mathrm{x}$ & & $\mathrm{X}$ & IV \\
\hline EB Enrollment & & $\mathrm{x}$ & & & IV \\
\hline Resources & & & $\mathrm{x}$ & & DV \\
\hline Strategies & & & $\mathrm{x}$ & & DV \\
\hline LATS & & & $\mathrm{x}$ & & $\begin{array}{l}\text { IV/ } \\
\text { DV }\end{array}$ \\
\hline
\end{tabular}

The "IV/DV" column indicates if the variable is an independent variable or dependent variable. The LATS variable, is used as an independent variable when asking if the disposition of teachers influences the resource and strategy use of teachers (question 3) or it can be a dependent 
variable when factors affect a teacher's disposition toward his or her emergent bilingual students (questions 1).

Study variables. The literature review in chapter two provides additional support for the ways in which the independent variables listed in Table 2 have been explored in the previous studies. It thereby provides an argument for their inclusion in this study.

The purpose of collecting and analyzing qualitative data first is to use grounded theory to determine what patterns can be observed from the data. These patterns provided insight into the dispositions of teachers, the factors that influenced these dispositions, and how they interacted with the choices that teachers make. This also means that additional independent variables that require subsequent analysis than were originally planned for were observed. For example, it was through the qualitative data analysis phase that I identified that the effective teaching strategies for emergent bilingual students that teachers use could be considered JGT. It made me question what techniques teachers use specifically for the linguistic and cultural needs of these students. This led to coding data for JGT and LRT strategies and ultimately their incorporation into the survey instrument that will be outlined below.

Eight of the independent variables listed in Table 2 were measured in relation to the dependent variable of teacher dispositions toward emergent bilingual students. This dependent variable will be quantified using the Language Attitudes of Teachers Scale (LATS) instrument developed by Byrnes and Kiger (1994). The LATS survey measured the teacher participants' dispositions toward emergent bilingual students using the summative score on a five-point Likert-type scale of 13 questions. The dependent variable is, therefore, an interval-level of measurement. 
Resource use and teaching strategy use are also dependent variables in question 2. For this question, I determine whether or not the teachers' dispositions impact the resources teachers use or the strategies they employ. I also use reports of current enrollment of emergent bilingual students in the classroom influences their use of resources and strategies.

I must note that for the LATS score, teacher resource scores, and teacher strategy scores, I am using Likert-type scale questions. Responses to such questions typically provide ordinallevel data. Through the procedure outlined above, I have taken the ordinal level data and converted it to interval level data. The assumption made of interval level data is that it must be evenly distributed. Likert-type questions, cannot be evenly distributed, creating skewness, which violates that assumption. However, Norman (2010) argued that, "ANOVA and other tests of central tendency are highly robust to things like skewness and non-normality" (para. 20). Using this data for a regression analysis may not provide the robustness. Similar to the ANOVA data, Norman (2010) argues that "all parametric tests we have examined, [are] extremely robust with respect to violations of assumptions" (para. 28). Therefore, while there is criticism regarding the use of ordinal level data converted to interval-level data, using the data in this manner is supported in the literature.

Survey instrument. Teacher dispositions toward emergent bilingual students primarily functioned as the dependent variable of the quantitative phase of the study. The existing literature on teacher attitudes toward emergent bilingual students includes both quantitative and qualitative data. However, for operationalizing this construct, I used the LATS survey developed by Byrnes and Kiger (1994), which measures the degree to which teachers look favorably or unfavorably upon emergent bilingual students. 
The LATS scale is a summative score of 13 Likert-type questions, some of which have been reverse coded, with a higher score indicating a less favorable attitude toward emergent bilingual students (Byrnes and Kiger, 1994). The authors developed the questions for the scale through the process of teacher interviews, reviewing literature on teacher, parent, and child attitudes toward language, and measures from race and ethnicity literature (Byrnes and Kiger, 1994). In the development of this tool, Byrnes and Kiger (1994) distributed the survey to 191 teachers in Arizona, Utah, and Virginia with 28 of these teachers participating in a retest to determine a test-retest reliability coefficient of 0.72 (Byrnes \& Kiger, 1994). According to Muijs (2011), "above .7 is usually considered to offer reasonable reliability for research purposes" (p. 63). The scale mean was $33.1(\mathrm{SD}=7.66)$ and the reliability coefficient of the scores was 0.81 (Byrnes \& Kiger, 1994). Using oblique rotation in their factor analyses, Byrnes and Kiger (1994) found that there were three groups of factor loadings, which the authors identified as language politics, LEP intolerance, and language support (Byrnes \& Kiger, 1994).

The LATS instrument has been previously used in the quantitative literature on language attitudes of teachers (Byrnes et al., 1993; Hutchinson, 2013). Stanoscheck Youngs and Youngs, Jr. (2001) were able to reduce the LATS survey to only two questions. In the survey they distributed to 224 teachers (148 valid surveys providing a response rate of 66\%) they first ask, "If you were told that you could expect two or three ESL students in one of your classes next year, how would you describe your reaction?" and secondly, "How would you describe your overall reaction to working with ESL students in your classroom?" The authors argued that they could reduce the LATS survey items to only these two questions due to the validity test run by Byrnes and Kiger (1994). Byrnes and Kiger (1994) determined the construct validity of their survey by measuring the LATS score in relation to an item that asked, "In general, how do you 
feel about having children in your classroom who speak little or no English?” (Byrnes \& Kiger, 1994, p. 231). Byrnes and Kiger (1994) found "the intercorrelation between the "willingness to have an LEP student in class' and the LATS scores was $r=-.62$ " (Byrnes and Kiger, 1994, $p$. 231). Muijs (2011) suggests that $r=0.7$ would be a strong intercorrelation. However, Stanoscheck Youngs and Youngs, Jr. (2001) used this correlation to develop two questions they believed would be "similar to" the LATS item asking about how teachers would feel about having an emergent bilingual student in his/her classroom, rather than administering the full LATS survey instrument. However, due to the current political climate in the United States, I disagree with this method for the quantitative phase of data collection. As mentioned, Byrnes and Kiger (1994) used oblique rotation to discover that their survey questions were related to language politics, LEP intolerance, and language support. I believe that in the current political climate, questions related to language politics are an important component to the LATS that should not be overlooked.

Stanoscheck Youngs, and Youngs, Jr., (2001) conducted $t$-tests and an ANOVA to examine five independent variables in relation to the attitudes reported by teachers. The independent variables included general educational experiences, ESL training, personal contact with diverse cultures, ESL student contact, and demographic characteristics. They then analyzed the data using a multiple regression analysis to determine if predictions could be made regarding the experiences of teachers and their attitudes toward emergent bilingual students. While statistically significant results were found for the ANOVA results indicating a relationship can be determined between the independent variables and teacher attitudes, the authors did not find statistically significant results with the regression analysis, which means that a causal relationship cannot be determined. 
A second study that used the LATS instrument more recently was Hutchinson (2013). In this study, Hutchinson (2013) administered the LATS survey in a pre- and post-test format to 25 pre-service teachers and analyzed results using a $t$-test. The survey was assigned to the students along with research papers in order to determine attitude changes within the population after a course in teaching emergent bilingual students. Hutchinson (2013) found that while teachers demonstrated more tolerant attitudes toward emergent bilingual students, the post-test also reflected a slight shift to more conservative language politics following a course on teaching emergent bilingual students. As the Hutchinson (2013) study demonstrates, data on language politics provides an additional source of information that may prove to be useful in the study. I did, however, incorporate the two questions used by Stanoscheck Youngs and Youngs, Jr, (2001) into interviews so that I could consider the teacher's dispositions toward emergent bilingual students as I analyzed the qualitative data.

For my proposed study, I used a three-section survey. The first section will make use of the LATS survey developed by Byrnes and Kiger (1994). For this section, I maintained the original LATS survey (Byrnes \& Kiger, 1994) as much as possible. I made minor changes, such as using the term English language learners (ELL) rather than limited English proficient (LEP) students, as was used by Byrnes and Kiger (1994). The term ELL is both less a reflection of deficit-based ideology and also more familiar to the teachers participating in the survey.

In addition to the first section of the survey, the LATS instrument (Byrnes \& Kiger, 1994), my survey contained two additional sections. The second section of the survey collected data on a teacher's use of resources. This section consisted of one question that was written in the form of a table so that teachers could answer how often (never, rarely, monthly, weekly, daily) they use various resources. The resources were selected from those reported by teacher 
participants in the qualitative phase of data collection. Nine resources were reported which represented three resources each among the three categories according to Cohen et al.'s (2003) theory. Again, these three categories of resources are environmental, conventional, and personal (Cohen et al., 2003). Space was provided for teachers to add other resources that they use that were not included in the survey. Six teachers offered additional resources other than those listed and these were then coded under the three aforementioned categories of resources. These resources included parents of emergent bilingual students, online forums and other online resources, ESL endorsement cohort materials, ESL teacher, French teacher, Google Translate and technology. One participant listed assistance from other emergent bilingual students and another mentioned storytelling or "acting out" cognates, realia, and total physical response (TPR). In this study, these items are considered strategies and was therefore not included in the data analysis.

The final section of the survey included questions related to demographics. Demographic questions have been added at the end of the survey so that I can analyze data to determine some of the factors that may contribute to shaping a teacher's disposition toward their ELL students. Furthermore, the demographic questions provide an opportunity for descriptive statistics to offer insight about the participants of the survey. This third and final section of the survey was based upon factors that the literature review indicated may impact a teacher's disposition toward emergent bilingual students. This section includes questions related to the independent variables of the study. These include questions about travel experience, teaching experience, and training in working with emergent bilingual students. Table 2 lists these variables and provides the level of measurement for each variable. The survey that teacher participants took in this study can be found in Appendix F. 


\section{Mixed Methods Approach}

Mixed method studies allow researchers to mix two or more methods of research to answer the same questions. Yin (2014) writes, "As such, mixed methods research can permit researchers to address more complicated research questions and collect a richer and stronger array of evidence than can be accomplished by any single method alone" (pp. 65). The research questions under consideration for this study lent themselves to both a quantitative and qualitative methodology. Therefore, the present study utilized such a mixed method design in a sequential format that began with a qualitative phase utilizing a case study design that drew heavily from grounded theory. The study concluded with a quantitative phase that will seek to generalize findings.

According to Creswell and Plano Clark (2011), there are several reasons why a mixed methods approach may be appropriate for one's proposed study. For this study, using a mixed methods design is a good fit because it allows for a greater understanding of the research questions by providing complementary information in both a general and more specific format. The mixed methods approach also offers a better opportunity to understand the data as it interacts with the theory of resource use developed by Cohen, Raudenbusch, and Ball (2003).

For this research study, I sought to understand the connection between teacher disposition and the resources that they employ in working with their emergent bilingual students as resources, as defined by Cohen et al.'s theory (2003). While I included questions on the survey that are related to teacher resources, it would be impossible to include all possible resources. Furthermore, teachers reported using resources that I had not considered. Classroom observations, lesson plan analysis, and semi-structured interviews were able to tease out such 
resources. In this sense, the use of a mixed methodology allowed me to better employ the categorizations of resources by Cohen et al. (2003).

Creswell and Plano Clark (2011) describe six different designs for mixed methods research. These include convergent, explanatory, exploratory, embedded, informative, and multiphase (Creswell \& Plan Clark, 2011). Of these six types of mixed methods research, I utilized the exploratory design. The exploratory design is one type of mixed methods approach that follows sequential timing. According to Creswell and Plano Clark (2011), "sequential timing occurs when the researcher implements the quantitative and qualitative methods in two distinct phases, with the collection and analysis of one type of data occurring after the collection and analysis of the other type" (p. 416). In the case of this study, the qualitative data collection preceded the quantitative data collection.

The exploratory design provided a strong fit with my research questions. To my knowledge, there are no existing theories regarding the dispositions of teachers toward emergent bilingual students and the influence these dispositions have on teacher resource use and strategies. Therefore, the exploratory design will be the most appropriate approach. In the exploratory design, research follows a qual $\rightarrow$ quan format. The qualitative data collection and analysis starts the research process so that a theory can be generated. For this phase I used grounded theory to analyze interviews, classroom observations, and documents. The research then proceeded to a quantitative phase of data collection to generalize the initial findings (Creswell \& Plano Clark, 2011). Creswell and Plano Clark (2011) recommend using different participants for the two research strands. They argue, "Because the purpose of the quantitative phase is to generalize the results to a population, different participants are used in the quantitative follow-up stage than in the initial qualitative phase" (Creswell \& Plano Clark, 2011, 
p. 188). However, because the focus of this study is one particular school district, it would be an inappropriate to make generalizations to the larger state, nation, or world context. Additionally, the population of the school district as a whole is only approximately 4,300 students. Therefore, every teacher, including those who participated in the qualitative phase, will be invited to participate in the quantitative phase of data collection in order to get a larger sample size for analysis purposes.

\section{Research Context and Study Sample}

\section{Unit of Analysis: School District Context}

The school district at the center of this study is located in a small Midwestern city with a population of approximately 30,000 . The majority of residents (77\%), are White, $13 \%$ are African American, and 8\% are Hispanic. The median household income in 2014 was $\$ 33,369$ (Cubit Planning, Inc., n.d.). The population and income has been on a steady decline for several years (Cubit Planning, Inc., n.d.).

However, while the general population of the city decreased, the immigrant population, particularly families from French-speaking West Africa, is increasing (Ward, 2013). An article published in 2013 estimated that at the time the immigrant population had increased 300 percent (Ward, 2013). According to American Fact-Finder, the foreign-born population of the city in 2013 was 1,106 . In the most recent survey completed in 2016, the number of foreign-born residents is 1,301 (2018). This indicates that the number of immigrants in this target city continues to rise. At one point, literacy and ESL services were provided to adults free of charge through the community college. While there was some disruption of services, adult ESL services are now provided for free by volunteers at various locations throughout the city and for a fee through the regional office of education. 
The school district is composed of one state-funded preschool program, five elementary schools, two junior high schools, and one high school. In 2017, there were 4,457 students in the school district served by 297 teachers (Illinois State Board of Education, 2017). 62\% of students are low-income and 4.4\% are English language learners (ISBE, 2017).

In recent years, the school district has struggled recently with budgeting troubles. According to an article published in the local newspaper in 2016, "Since 2009, [the district] has received $\$ 6.8$ million less general state aid than it was promised" (Zigterman, 2016b, para. 13). As a result of the on-going budget shortfall, the school district experienced a reduction in work force that included 16 teachers and 13 aids losing their jobs without being called back (Zigterman, 2016a). Despite the recent cuts, the school district has struggled with finances. Depending upon passing a proposed levy, the school district is expecting a budget deficit between $\$ 1.2$ million and \$2.3 million (Zigterman, 2016a).

At the time of writing, the school district currently serves 193 students who speak languages other than English in the home and are receiving services for English as a second language (ESL). While 11 different languages are spoken within the school district, the primary languages represented in the district are Spanish and French/Lingala. The school district offers a Transitional Program of Instruction (TPI) program in most buildings. In this design, students who speak languages other than English are provided support in increasing their proficiency in the English language. One elementary school, also offers a Transitional Bilingual Education (TBE) program, which provides support in both English and the primary language of the students, which in this case is Spanish. Both designs provide students with in-class or pull-out instruction in English. 
This school district has not always been the home to a large number of emergent bilingual students. A meat-packing plant in a nearby community employs a large percentage of the French/Lingala-speaking families. The hiring process of this facility offers incentives for employees to find new employees, thereby increasing the certain populations in this area. While the immigrant families have mainly lived in that nearby city, more recently the immigrant families are moving to the town at the center of this study. As one document for the school district states:

These families make our community their home for a variety of reasons, [This city] has a surplus of rental housing, there is a strong networking system between families of the same language and cultural background, a religious affiliation and connection to church and a strong relationship and deep comfort level with our current ELL outreach family liaison. (Technical Assistance Document, n.d., p. 1).

Because the immigrant population has increased so rapidly over a short period of time, it is possible that it could decrease just as quickly. However, given the multifactorial reasons for moving to this area, it is likely that while there are jobs in the area for immigrant families, they will continue to live in this town.

The school district has undergone a dramatic increase in emergent bilingual students who receive ESL services. Seven years ago, there were only three students in the school district who received ESL services and all of these students spoke Spanish. Today there are 193 students receiving ESL services who primarily speak French and Lingala. This means that within a relatively short time frame, the district needed to quickly find teachers who are qualified to teach the emergent bilingual students, and preferably teachers who have a background in learning and 
speaking French and Spanish. Within this time period the ESL team has grown from one teacher to 13 teachers and one aid. The department is currently seeking to expand further.

This also means that teachers that have worked in the school district for longer than seven years, many of whom have also graduated from this school district, have seen their jobs as well as their community change dramatically in a very short period of time. One might expect that this change would been met with a variety of responses from the teachers.

\section{Procedure}

Qualitative data collection is the first phase of this sequential mixed methods design. Below I will provide a description of the process that I used to collect qualitative data. Following this description is the quantitative data collection process that I used. Both phases of data collection contributed to answering the research questions.

\section{Qualitative Data Collection Procedure}

All third through fifth grade teachers who had one or more emergent bilingual students in his or her classroom at the time of the study were invited to participate in the study. During the 2017-18 academic year there were 24 third, fourth, or fifth grade teachers with emergent bilingual students in their classrooms who were then invited to participate. Participants were selected based on pre-determined criteria. I looked for teachers who I, as a district employee, had not worked with in the past. That limited the group to 15. From there, I looked for teachers who had a variety of experience in terms of their years of teaching and whether they grew up in the school district community. During this research phase, I began data collection with an initial teacher interview, which can be found in Appendix E. Interviews were scheduled for the beginning and end of the qualitative phase of data collection. Interviews were offered to be held 
at a place at the teacher's convenience in a public location of their choosing. Every teacher chose to be interviewed in her own classroom.

I then observed each classroom two times to conduct classroom observations using the SIOP protocol (Appendix C). Observations lasted for one entire lesson period from start to finish. Prior to conducting the observation, I e-mailed teachers to remind them of my visit. This email also served to once again inform teachers of the purpose of the study and their voluntary participation. Teacher consent was obtained prior to the observation and consent forms were signed at the time of the first interview in order to limit the extent to which my observation would interrupt the classroom activities. I used a paper copy of the observation protocol along with the predetermined pseudonym stored in a locked filing cabinet. Participant pseudonyms were used in order to maintain confidentiality on the digital resources.

In the pre-observation email, I asked teachers to provide me with a copy of any teaching materials or assessments that the teacher would be using and would hand out to students throughout the lesson. I also asked teachers for a copy of their lesson plan for the particular lesson that was observed. I made it clear that the lesson plan did not need to be anything more than what they have recorded for their own lesson planning system. In addition to the lesson plans, the documents that teacher participants provided me for analysis included a copy of class reading materials, graphic organizers, and worksheets. Following the final observation, I interviewed teachers a second time. I then analyzed the qualitative data to look for themes and patterns. These themes and patterns were then used to modify the survey instrument that was distributed during the next phase of data collection. Of the three qualitative data collection methods (interviews, document analysis, and observations), interview data was placed at the fore of the study. Because interviews were semi-structured, they allowed the flexibility to ask 
clarifying questions regarding the choices teachers make in their resource and strategy use and their motivations behind these choices. Document analysis and observations provided data in what resources and strategies teachers employed, but not the reasons behind these decisions.

\section{Quantitative Data Collection Procedure}

Following the development of a theory from the qualitative data, I continued the study with the quantitative phase of research. This phase of data collection took the form of an online survey distributed to school district teachers. The survey used the LATS scale (Byrnes \& Kiger, 1994) to quantify teacher dispositions toward emergent bilingual students. The survey then asked about the resources and strategies that teachers employ and the frequency with which they are used. The survey concluded with questions regarding teacher characteristics that current research suggests may influence dispositions toward emergent bilingual students.

The online survey was developed using Qualtrics and was administered to all teachers using a district email list. A reminder email was sent exactly one week later. A final reminder was sent after one additional week. The original email and reminders informed participants that their participation is anonymous and voluntary and that they may choose to withdraw or stop the survey at any time. Teachers were asked to click a box to state that they understood their voluntary participation before they were allowed to begin the survey. While it is recommended by Creswell and Plano Clark (2011) that in exploratory mixed methods designs the participants of the qualitative study do not participate in the quantitative phase of data collection, I made the choice to keep these participants in the study. The argument that (Creswell \& Plano Clark, 2011) make is that the qualitative data are used to develop theory. Therefore findings from the quantitative analysis may show bias (Creswell \& Plano Clark, 2011). However, my decision was to keep these participants included in the quantitative phase of data collection primarily because 
finding teachers within the school district total population who has significant travel experience or who learned English in an ESL program, as my qualitative teacher participants reported, may be difficult to find. Qualitative participant, Linda, has significant travel and international experience. I would like to examine how such experience impacts teacher dispositions, resources, and strategies. While I may not have a significant population of teachers who have this level of experience, the more participants I have who participate in the study with this experience, the better the analysis of this variable will be. I then analyzed the survey data using $t$-tests, ANOVA, descriptive statistics, and linear regression. Finally, the data from the two phases will be considered together to answer the research questions.

\section{Combining Qualitative and Quantitative Data}

The qualitative and quantitative data collection and analysis were designed to complement one another. The first phase of data collection, which is the qualitative phase, used interviews, observations, and document analysis to determine what factors influence an individual teacher's disposition toward their emergent bilingual students, as well as the interplay between the teacher's disposition and his or her teaching strategies and use of resources.

The findings from the qualitative data collection was then used to modify the survey that was sent to teachers during phase two. The purpose of the survey in the quantitative phase of data collection was to determine if the findings from the qualitative phase of data collection can be generalized to the larger school population. I did this by considering if the patterns I found in the qualitative phase of data collection match the results of the data analysis conducted in the phase two quantitative phase. Secondly, the quantitative phase helped to determine if there is any relationship between the independent and dependent variables. 


\section{Data Analysis}

As per the requirements of an exploratory, sequential mixed methods design, I first analyzed qualitative data before looking to the quantitative data collection and analysis. Table 3 depicts how data were analyzed to answer the research questions. Below that table is a description of how I analyzed both data sets to arrive at the answers to my research questions. Table 3

Data Analysis by Question

\begin{tabular}{|c|c|c|c|c|c|c|}
\hline \multirow[t]{3}{*}{ Question } & \multicolumn{2}{|c|}{ Qualitative Analysis } & \multicolumn{4}{|c|}{ Quantitative Analysis } \\
\hline & & & Descriptive & & & Linear \\
\hline & Inductive & Deductive & Statistics & $t$-test & ANOVA & Regression \\
\hline 1 & $\mathrm{x}$ & & & $\mathrm{x}$ & $\mathrm{x}$ & \\
\hline 2 & $\mathrm{x}$ & $\mathrm{x}$ & $\mathrm{x}$ & & & \\
\hline 3 & $\mathrm{x}$ & & & & & $X$ \\
\hline 4 & $\mathrm{x}$ & & & & & \\
\hline
\end{tabular}

\section{Qualitative Data Analysis}

To analyze the data, I first needed to process it into a form that was conducive for review and analysis. Observations included information provided from the SIOP checklist as well as observation notes. Document analysis included the documents provided by the teacher and the analysis protocol created for this study and found in Appendix D. Finally, all interviews were recorded and transcribed.

Grounded theory is defined as "a form of qualitative research developed by Glaser and Strauss (1967) for the purpose of constructing theory grounded in data" (Corbin \& Strauss, 
2015). Although I am using resource theory as a conceptual framework to aid in categorizing the types of resources that teachers call upon in their work with emergent bilingual students, I drew from grounded theory to develop a theory concerning why and in what way teachers call upon these resources and teaching strategies. I considered how teacher disposition factors into their pedagogical decisions. To my knowledge, no theory exists on the connection between the attitudes of teachers toward emergent bilingual students, the resources they choose to draw upon and the strategies they choose to employ with these students. The goal of this research is to go beyond describing the circumstances of teacher disposition to develop a theory that explains teacher pedagogical choices in working with their emergent bilingual students that connects resources and strategies with dispositions. Therefore, as grounded theory is concerned with the development of new theory (Corbin \& Strauss, 2015), such a methodology proved to be appropriate for analyzing the research questions.

The qualitative data was analyzed in different ways depending upon the research question that I was attempting to answer. I followed an inductive approach to coding data in analyzing responses that pertain to the dispositions of teachers toward their emergent bilingual students (research question one), why they decide to use particular resources or pedagogical strategies (research question three), and the interplay between resources and strategies (research question four). The theories related to best practices for teachers of emergent bilingual students and teacher resources were used to guide analysis only in terms of identifying resource and strategy use and for interpretation of inductive findings.

In the coding phase, I used the process of open, axial, and selective coding outlined by Corbin and Strauss (2015) to search for relevant themes and development of theory. That is, to conduct open coding, I read the interview transcripts, SIOP instrument, observation notes, and 
document analysis checklist several times while using memos to help identify and describe codes as they come up. As I read the data, I assigned a code or label to each concept that stood out, regardless of whether or not it initially appeared to apply to the research questions. I recorded codes and labels in memos as they were identified. In the phase of axial coding, I again reread the transcripts and wrote memos to identify and compare information filed under the same code. At this stage, I considered the relationship and connections between the data. During this phase, all open codes had been assigned and memos had been recorded for the initial set of codes. I then organized open codes into clusters of larger ideas, which became my initial themes. Finally, during the phase of selective coding, I considered the context of the information I coded and the core of the research question. During this final stage in coding, I reread the interview transcripts, observation information, and document analysis checklists to determine how the new perspective on context answer the research questions (Corbin \& Strauss, 2015).

As an example, the label "Who is an emergent bilingual?" was applied to several lines of data and identified early in the coding process. Teachers repeatedly asked if a student "counted" as an emergent bilingual student, particularly if the child's social language proficiency was at a higher level. I continued to identify this code within the data as teachers described their understanding of when to differentiate for emergent bilingual students. Typically, teachers more often described that they do not differentiate for their emergent bilingual student when they perceive that student to have a higher English proficiency level. This code and concept continued to appear in observation notes regarding teacher selection of student for whom they provided additional support and in the document analysis for lesson plans as well. Later, in reviewing all codes, I realized that the teacher's concept of what constitutes and emergent bilingual student was related to another code, that is, the teacher's understanding of the student's home language 
and culture. It was also related to the teacher's understanding of the student's background. These three codes together were then collapsed under the larger theme of the teacher's knowledge of the student.

The second research question regarding the resources and strategies that teachers call upon in their work with emergent bilingual students, was analyzed using a hybrid of inductive and deductive codes. By this I mean that I used existing codes (deductive coding) while also considering new patterns in the data (inductive coding). Interviews, observations, and document data were coded into categories for resources and strategies. Resource codes were derived from Cohen, et al. (2003) for personal, environmental, and conventional resources. For teaching strategies, I looked specifically for effective strategies in the area of assessment, culture, parent/family involvement, and instruction. However, I was also open to new categories of data that may develop through the use of inductive coding.

While the use of deductive codes was helpful, I found through interviews that when asked what pedagogical strategies teachers use specifically for their emergent bilingual students, teachers often provided their answer and followed this up with a common statement. That is, teachers often reported what practices they use that they feel are effective for this group of learners and then often added, "but that's something I do for all students." Due to this response pattern, I returned to the literature to look at teaching strategies that are useful for all students and also those specifically for emergent bilingual students. This discovery brought me to the inclusion of JGT and LRT strategies in the literature review. I then returned to the interviews, observations, and document analysis and coded for JGT and LRT strategies.

Data saturation. For this study, the qualitative phase of data collection took place in the spring semester of the 2017-18 school year. The second interview allowed for the teachers to 
again restate the resources they turn to, confirm the strategies that they employ, and to verify their disposition and/or philosophy toward educating emergent bilingual students. I identified that saturation had occurred when no new themes emerged and no new data appeared. In the second interview I revisited teacher dispositions and the resources and strategies that teachers used or that I observed. When these were revisited, I knew that data saturation had occurred when teachers provided the same resources and strategies that had already been discussed or observed and restated previously mentioned answers regarding their dispositions towards their emergent bilingual students. Several teachers prefaced the answer to these questions with phrases such as, "I think I had mentioned earlier that..." (Sephanie, Interview 2) or "Well, as I said before..." (Linda, Interview 2).

Trustworthiness. Lincoln and Guba (1985) suggest that the researchers ask themselves four questions for verifying trustworthiness:

1. "Truth value": How can one establish confidence in the "truth" of the findings of a particular inquiry for the subjects (respondents) with which and the context in which the inquiry was carried out?

2. Applicability: How can one determine the extent to which the findings of a particular inquiry have applicability in other contexts or with other subjects (respondents)?

3. Consistency: How can one determine whether the findings of an inquiry would be repeated if the inquiry were replicated with the same (or similar) subjects (respondents) in the same (or similar) context? 
4. Neutrality: How can one establish the degree to which the findings

of an inquiry are determined by the subjects (respondents) and conditions of the inquiry and not by the biases, motivations, interests, or perspectives of the inquirer? (p. 290).

These questions are all a matter of trustworthiness. The authors provide several methods by which researchers can increase the trustworthiness of their work. Each of these methods will be defined below. Following the definition, I will discuss how I used each method in my own research.

Prolonged engagement. "Prolonged engagement is the investment of sufficient time to achieve certain purposes: learning the 'culture,' testing for misinformation introduced either by distortions either of the self or of the respondents, and building trust" (Lincoln \& Guba, 1985, p. 301). In order to achieve prolonged engagement, I observed each classroom two times throughout a three-month time period. With six cases, I had 2 observations each. I used two different interviews as well as analyzed multiple documents for each case that was examined. Finally, because at the time of the qualitative data collection phase I was a teacher in the school district of the study, the time that I had spent getting to know the teachers, families, and policies within this school district as a part of my job had helped me to understand the culture, which is one essential component of prolonged engagement.

Persistent observation. Lincoln \& Guba (1985) suggest that "the purpose of persistent observation is to identify those characteristics and elements in the situation that are most relevant to the problem or issue being pursued and focusing on them in detail" (p. 304). The authors wrote that it is through persistent observation that the study achieves depth. This study used two observations for each case. Again, this was a total of 12 observations within three months. While these two observations may not constitute persistent observation, it accounted for approximately 
two hours of observations for each teacher. I recorded observation notes and utilized the SIOP instrument for consistency between the two observations. I asked teachers follow-up questions pertaining to the observations during the second interview. Finally, following the last interview I used member-checking procedures to send an email to teachers asking them to verify results.

Triangulation. Along with prolonged engagement and persistent observation, triangulation is the third method that Lincoln and Guba (1985) suggested for increasing the production of study credibility. Lincoln and Guba (1985) described triangulation as "the use of different sources, methods, investigators, and theories" to ensure that findings from the data are credible and valid (p. 305). For this study I used multiple sources (document analysis, observation, and interviews). I also used multiple methods (a quantitative approach to analyze variables and a qualitative approach that used grounded theory and drew heavily from a case study design). In this way, I am better able to arrive at credible conclusions than if I had only used one method or one data source.

Peer debriefing. Lincoln and Guba (1985) stated that peer debriefing "is a process of exposing oneself to a disinterested peer in a manner paralleling an analytic session and for the purpose of exploring aspects of the inquiry that might otherwise remain only implicit within the inquirer's mind" (p. 308). As a doctoral student, my dissertation committee chair helped me to consider and talk through the thoughts on the data analysis as they arose. However, I recognize that Lincoln and Guba (1985) argued that the "the debriefer should be neither junior-lest his or her inputs are disregarded-nor senior-lest his or her inputs be considered mandates, or lest the inquirer 'hold back' for fear of being judged incompetent" (p. 309). I am aware that my dissertation committee and dissertation chair do not meet these requirements. Therefore, I also set up monthly meetings with a fellow doctoral student familiar with current research on 
emergent bilingual students. I also used personal memos throughout the dissertation writing process, particularly through the data analysis phase in order achieve some of the same goals Lincoln and Guba (1985) suggested can be achieved from peer debriefing; that is, memos helped clarify my thoughts, consider personal biases, test working hypotheses, consider my next steps, and consider feelings and emotions that may cloud my judgment.

Member checking. "The member check, whereby data, analytic categories, interpretations, and conclusions are tested with members of those stake holding groups from whom the data were originally collected, is the most crucial technique for establishing credibility" (Lincoln \& Guba, 1985, p. 314). At the end of my qualitative phase, I provided each of the case study participant teachers an opportunity to review the main points of my findings. This opportunity focused primarily on what resources they drew upon most, what strategies they use most frequently, connections between the resources and the strategies that they use, and their general perspectives toward their emergent bilingual students. For this study, member checking came in the form of an email at the end of the data analysis period. The member checking email included data such as the number of years they have been teaching, number of emergent bilingual students they have taught, and other facts that I asked them to verify. I also included themes related to resources and strategies. All teachers verified the information was accurate and one teacher provided additional strategies that she uses at that time. At that time, I offered teacher participants the ability to correct any errors that they noticed and offer any additional information that they felt would be relevant to the study.

\section{Quantitative Data Analysis}

Quantitative data were analyzed in relation to only the first three research questions, namely: 
1. What influences the dispositions that teachers hold toward their emergent bilingual students?

2. What resources and strategies do teachers draw upon specifically for working with their emergent bilingual students? And,

3. How does a teacher's disposition inform the resources and strategies they draw upon in working with emergent bilingual students?

For the first research question and the accompanying seven sub-questions, nominal and ordinal level independent variables of teacher characteristics were analyzed in relation to the interval level dependent variable of disposition as measured by the LATS score. This analysis was completed using a $t$-test or an analysis of variance (ANOVA). The $t$-test is designed to "compare means of a dependent variable between two groups" (Muijs, 2011, p. 111). Using a $t$ test for the nominal variables in this study, I was able to compare the mean of the summative LATS score to the nominal independent variables from the survey mentioned above (Muijs, 2011). The $t$-test is set up to compare the means of two groups when the independent variable has two categories of responses (Muijs, 2011). There was only one sub-question requiring a $t$ test. These questions ask "Have you ever been in a country where you did not understand the language?" for which the only two answers are either yes or no.

For questions in which I need to compare the means of dependent variables with three or more categories, an ANOVA is a more appropriate test (Muijs, 2011). Muijs (2011) describes the ANOVA as "a method that allows us to compare the mean score of a continuous (or ordinal variable with many scale points) variable between a number of groups" (p. 175). The ANOVA is designed to compare the means of many groups in order to determine if the variance between the spread of each group is larger than would be predicted (Muijs, 2011). These remaining nominal 
and ordinal level variables describe the teacher's bilingual status, time spent in a country in which they did not understand the language, time teaching, time teaching within the target district, training for emergent bilingual students, and the number of emergent bilingual students the teachers have worked with in his or her career. Each of these response groups sort participants into more than two groups which make the $t$-test impossible and the ANOVA the ideal statistical analysis for this question.

The second research question seeks to determine the resources and strategies that teachers use with emergent bilingual students. Descriptive statistics have been employed to analyze this data. In chapter four, I report on the most commonly used type of resource (personal, environmental, and conventional) and the most commonly used teaching strategies (JGT or LRT).

In order to quantitatively analyze the third research question, I needed to process the survey data. I quantified the amount that a teacher reported using the data by assigning a value of 5 when a teacher reported using that resource daily, 4 for weekly, 3 for monthly, 2 for rarely, and 1 for never. I then calculated the sum of the teacher's use of each of the different resource types (personal, environmental, and conventional). I was able to do the same for teaching strategies as divided into the categories of JGT and LRT. Therefore, the resource use and strategy use became an interval-level variable. Because I examined an interval level dependent variable (the LATS scale) and an interval level independent variable (number of each resource teachers use with emergent bilingual students and the number of each type of strategy teachers use with emergent bilingual students), I used a linear regression to determine if there is a predictive relationship between the two variables. A linear regression can be used to determine the causality of the independent variable on the dependent variable (Muijs, 2011). 


\section{Limitations}

There are several limitations for this study. These limitations include the fact that the data is self-reported by teachers; the data is collected from only one school district and may be difficult to generalize findings to a wider population; the emergent bilingual students primarily come from one racial group and national origin making separation between disposition toward emergent bilingual students difficult to separate from disposition toward a particular race or national culture. In qualitative research, the positionality of the researcher may be one major limitation. Therefore, I will examine my positionality in detail. Following this section, I will describe the limitations that I previously listed.

\section{Positionality}

Researcher bias must be considered regardless of whether the study follows a quantitative or qualitative design. In quantitative research we must try to leave all bias out of our work. In qualitative research, bias cannot be avoided. Some strategies for reducing bias can be useful in both qualitative and quantitative studies. For example, researchers can achieve this by ensuring that research questions are worded in a way that does not presuppose or lead to specific answers (Miujs, 2011). Surveys can be reviewed by other researchers and pilot surveys can be sent out. (Chenail, 2011; Miujs, 2011). However, in order to best address the bias that is inevitable in our research the investigator must consider what their bias or biases may be and plan for ways to reduce the bias in their research. Below is a discussion of the aspects of my past that may lead to bias in my research.

Buckle (2009) writes,

Whether the researcher is an insider, sharing the characteristic, role, or experience under study with the participants, or an outsider to the commonality shared by participants, the 
personhood of the researcher, including her or his membership status in relation to those participating in the research, is an essential and ever-present aspect of the investigation (p. 55).

Therefore, to maintain the quality of this research, I need to consider my status as an insider or an outsider while pursuing this research. I hold an insider perspective in some areas of this research an outsider perspective at other times.

My research considers the way teachers, who in this particular district are primarily White, monolingual, and female, work with their students who are immigrants or children of immigrants. I will discuss first the manner by which my experiences and perspectives offer me outsider status in relation to the teacher participants. I will follow this with a discussion on how my experiences may also provide me with insider status in relation to the teacher participants.

First, as mentioned, this research is focused on immigrants and children of immigrants. As a daughter of an immigrant myself, this issue is important to me. I am very familiar with the challenges of living in and raising children in a foreign country. I have personally watched my mother frequently experience challenges presented by living in an unfamiliar culture and listened to my mother express frustration from these challenges on many occasions.

Furthermore, many of the students in this school district are of West African descent. This is the fastest growing population in the school district. Therefore, any discussion of emergent bilingual students with teachers usually involves a discussion of this particular group. I am a Returned Peace Corps Volunteer. For two years I lived in West Africa in a Gambian village, first with a Mandinka family and then with a Fula family. Again, this means that the West African population is quite close to my heart. I feel that I have a strong understanding of Gambian culture and great empathy for the people of Africa in general. 
I believe that the time I spent living abroad helped me to understand and empathize with the difficulties of adjusting to a new country and culture. It is my belief that no matter where you come from and where you are going, you miss the familiarity of people, places, food, sounds, and smells of home. Sometimes constantly being in an unfamiliar environment is exhausting and frustrating.

I have also tried at several points in my life to learn a foreign language. To various degrees and with various levels of success, I have studied French, Spanish, Mandinka, Pulaar, and Lingala. It is through this process that I believe I developed great understanding of the challenges that learning a foreign language present. I am familiar with feeling stupid because I could not pick up on what others were saying. I will always hold with me the memory of how I felt when one Gambian woman told me that my "mind is not sweet," a Gambian way of saying I was unintelligent, because I could not speak Pulaar.

Finally, I also have experience as an ESL teacher. In this role, have seen the frustrations of students who struggle to understand the English language and the content of their subjects at the same time while only limited modifications have been made by teachers. I have become frustrated myself when I see my students in their general education classrooms given a coloring page while other students work on content-related materials. The teacher participants may not view me as an insider, a fellow teacher, but rather an outsider in the fact that while I was a teacher within the district during the qualitative phase of data collection, I am not a classroom teacher. I had a distinct set of challenges on the job than what the classroom teachers experience.

The factors listed may bias me toward an overly empathetic view of the students' experiences. These factors may also contribute to an outsider perspective as I conduct research and analyze the data. However, I still possess characteristics that are similar to the teachers of the 
school affording me an insider status. Like the majority of teachers in this school district, I am a White, female, American, and a native-American English speaker. To a large extent, I understand American culture and the expectations of teachers in the American school system.

One final aspect of my positionality to consider in this research is the fact that I work in the school district where the study is taking place. During the qualitative phase of data collection, I was working as an ESL teacher for the district. After this phase of data collection was completed, I changed job assignments and began working as an elementary school building principal during the quantitative phase of data collection. As a result, I have insider information on some of the challenges that the teachers face and as well as the practices of some of the teachers.

Corbin Dwyer and Buckle (2009) write, "The benefit to being a member of the group one is studying is acceptance. One's membership automatically provides a level of trust and openness in your participants that would likely not have been present otherwise" (p. 58). It is possible that the teachers who participated with this study viewed me as an insider as fellow White, female teacher within the school district and were more willing to open up to me about their experiences. However, Corbin Dwyer and Buckle (2009) caution that insider status may lead to assumptions by the researcher. These assumptions may influence the manner by which the research is carried out and the way that the data is analyzed such that the focus is more on shared experiences between researcher and participant and neglect to the individual experiences of the participants (Corbin Dwyer \& Buckle, 2009). I needed to be aware that my assumptions about teachers does not color the way I analyze data. I attempted to achieve this objective through peer debriefing with a non-district fellow doctoral student and the use of memos throughout the data collection process. 
In order to mitigate the quality control concerns associated with insider vs. outsider status in my qualitative research, I engaged in a process of reflexivity. Berger (2015) writes, "Reflexivity is commonly viewed as the process of a continual internal dialogue and critical selfevaluation of researcher's positionality as well as active acknowledgement and explicit recognition that this position may affect the research process and outcome" (p. 220). Reflexivity is not a task to engage in one time. Instead, Guillemin and Gillam (2004) write, "Reflexivity in research is not a single or universal entity but a process - an active, ongoing process that saturates every stage of the research" (p. 274). In order to engage in the practice of reflexivity, I considered biases before engaging in data collection, wrote memos throughout the data analysis process, and participated in peer debriefing with a fellow doctoral student.

\section{Other Limitations}

This study presents some additional limitations as well as potential researcher bias. For the quantitative phase, I will primarily be able to run a $t$-test, an analysis of variables (ANOVA), and descriptive statistics. I will only be able to run a regression analysis that has the capacity to determine a predictive component between teacher resources and strategies and teacher dispositions. That means that looking for a correlation and making predictions will be beyond the scope of this study for many of the independent variables.

Secondly, the teacher survey data regarding dispositions is self-reported. While interviews and observations may help to form a better and more complete understanding of teacher dispositions, it is possible that teachers answer questions inaccurately. Similarly, it is possible that certain beneficial teaching strategies or resources will be overlooked.

In addition, the qualitative strand of research does not lend itself to generalizability of findings. While the quantitative strand will aid in the ability to generalize findings, the 
participants of the study come from one school district in a mid-sized town in the Midwest. The findings from this study may not necessarily reflect the dispositions or resource and teaching strategies that may be present in other parts of the country. Furthermore, the target population of the study is also fairly small because the surveys will only be distributed to the teachers of one school district. Again, a smaller sample size reduces the ability to generalize findings.

Finally, while the emergent bilingual population of this school district represents twelve different languages, the students are primarily of West African descent. Teacher dispositions, therefore, may be connected to the particular population of emergent bilingual students. Further studies may need to be conducted to determine if teacher dispositions change depending upon the population of emergent bilingual students present in the school district.

\section{Summary: Practical Implications of the Study}

Understanding what factors influence the dispositions of teachers toward their emergent bilingual students will help teacher preparation programs design educational experiences for preservice teachers that develops and fosters positive attitudes toward the multicultural classes these future teachers will serve. Furthermore, a study of the resources and strategies that teachers use will also inform pre-service teaching programs of what tools are available to teachers in the field. Any information on underutilized resources and strategies can be used to create professional development as it provides insight into what resources and strategies need to be highlighted for in-service teachers.

Adding to the body of literature on the experiences and characteristics of teachers who are more likely to have positive dispositions toward emergent bilingual students will help school district administrators make hiring decisions. Literature in the field of education suggests that students live up to teacher expectations (Jussim \& Harber, 2005; Sorhagen, 2013). Therefore, 
knowing the qualities of teachers with positive attitudes toward emergent bilinguals can be connected to finding the right teaching candidate to foster high academic achievement in linguistically diverse students. 


\section{CHAPTER IV: FINDINGS AND RESULTS}

In the following section, you will find the findings and results of the research questions. The names of all teachers, volunteers, and students have been changed in this dissertation to maintain privacy. I will present the qualitative findings first, followed by the quantitative findings, and concluding each research question section with the conclusions that can be drawn by combining the data sets. However, before these can be considered, it is useful to briefly review the participants of the study.

\section{Participant Review}

Holly is the youngest of the participating teachers with only three years of teaching experience. She currently teaches fourth grade at a school that pulls from a population of students at a lower socioeconomic level (School A). Her husband, a Mexican immigrant who arrived in the United States when he was in fourth grade, teaches that same grade level in a different school district. Her previous position within the school district as a school librarian gave her the experience of working with over 40 emergent bilingual students at a wide variety of proficiency levels.

Stephanie is a third grade teacher who has been teaching for 22 years. She works in a school that also pulls from a population typically at a lower socioeconomic level (School B). Stephanie has worked with emergent bilingual students at a variety of proficiency levels.

Jennifer is a third grade teacher at a school that draws students from families at a higher socioeconomic level (School C). She has taught emergent bilingual students at a variety of proficiency levels. 
Susan works at School A with Holly and also teaches fourth grade. At the time of the study, Susan was two years away from retirement and has been teaching for 39 years. She is also a board certified teacher.

Melody teaches third grade at School C with Jennifer. She has taught emergent bilingual students at a variety of proficiency levels. She has a long history in the school district and shared that she went to third grade and student taught third grade in the classroom where she was teaching at the time of the study.

Finally, Linda teaches fourth grade at School D, which draws students from families who are typically at a higher socioeconomic level within the school district. Linda immigrated to the United States in $6^{\text {th }}$ grade from Vietnamese where her family fled after facing much discrimination for being ethnic Chinese. Linda reports that she learned English through her school ESL program. She has taught students at a variety of proficiency levels.

\section{Qualitative Teacher Dispositions}

In order to understand what influences the dispositions that teachers hold toward their emergent bilingual students (question 1) or how dispositions inform the resources and strategies that teachers call upon (question 3), it is important to first identify the dispositions that teacher participants hold toward their emergent bilingual students. Therefore, the qualitative research findings section will begin with a determination of the teachers' dispositions. This will help in further analyzing the research questions.

Every one of the teachers who participated in the case study research expressed either a neutral or positive disposition toward working with emergent bilingual students. The dispositions of teachers were assessed using the two questions from Stanoscheck Youngs and Youngs, Jr., (2001) that were identified as accurate indicators of teacher's dispositions. These two questions 
were taken from the LATS instrument (Byrnes and Kiger, 1994). The questions ask, "If you were told that you could expect two or three ESL students in one of your classes next year, how would you describe your reaction?" and secondly, "How would you describe your overall reaction to working with ESL students in your classroom?” (Stanoschek Youngs \& Youngs, Jr., 2001, p. 108).

When asked directly about their dispositions toward emergent bilingual students, all teachers reacted positively, as I have recorded below. However, I then examined other statements made by the teachers to determine if, while feeling positively about their emergent bilingual students, there was evidence of explicitly positive statements about what their emergent bilingual students bring to the classroom or if there was evidence of deficit ideology.

I used these statements to group teachers by disposition. Teachers seemed to reflect either a positive disposition, neutral disposition, or a more nuanced disposition. I will discuss each of these groupings along with reasons for placing teachers within this category.

\section{Positive Disposition}

Positive dispositions are characterized as a genuine excitement for working with emergent bilingual students. They see the emergent bilingual presence in the town and in their classroom as a benefit to the community and classroom as a whole. They express a desire to learn about the home country and culture of their emergent bilingual students. These teachers either seem to have a better understanding of the principals of teaching ESL or they express an understanding of areas where they need to learn more.

Teachers with a positive disposition were Holly and Stephanie. Statements made by these teachers that provided evidence of their dispositions are included below. 
Holly. Holly's response to the first disposition question on her reaction to working with emergent bilingual students next year was:

Scared but excited. This is a very real reality for me next year and I am apprehensive because I don't want to fail them, but I am very excited to be their leader on their fourth grade journey and learn things from them along the way as well. (Interview 2)

In response to the second disposition question, she states:

I'm always a little nervous at first because I don't want to leave anyone out or make anyone feel uncomfortable. I want all of my students to feel welcome and like they belong and are valued and I'm always worried that I won't be able to convey that to my ESL students. I'm genuinely excited to have them though because they get to teach me and their classmates as well! (Interview 2)

At other points in the interviews, Holly stated, "I want to be able to share in [the emergent bilingual student's home] culture and have all my students realize I'm just as invested in where they came from as where they are" (Interview 2). She also states, "I love learning about culture it just makes me very happy and I love seeing different insights and perspectives and I would love to be able to talk with the students and get their input and stuff" (Interview 1).

It is apparent from these comments that Holly maintains a positive outlook of world cultures and wants to share this with all her students. Holly sees a personal interest in the home cultures of her students and is aware that she has a challenge in having to work harder to meet their needs.

Stephanie. Stephanie was also characterized as having a positive disposition based on the answers that she provided to the disposition interview questions and other information she provided throughout the interviews. 
In response to the question asking about her overall reaction to working with emergent bilingual students, she said,

My experience has been very positive and I think I've shared that last time. Some of it has been very eye-opening to me. I rethink how I teach, rethink that, where I stand, rethink how I say things. It's been so good for my other kids too and that's been so good to watch, to see them have compassion for others, to see them help others, to see them think about themselves. Also, it's been it's been wonderful for me. I've loved every minute of it. It's been scary at times when they come in and they're overwhelmed and they're scared and it hurts my heart because I don't know how to help them sometimes. (Interview 2).

At the end of the first interview, without prompting, Stephanie said, "I have to say, my Frenchspeaking students have been the highlight of my career. They really opened my eyes to so many things" (Interview 1).

There was no indication of deficit ideology in her interviews. When discussing students who were at the emergent stages of the four language domains, she was careful to say that they were low "English-wise." This indicates that she understands that a student's linguistic abilities are not an indication of their academic abilities. Stephanie spoke at length about her need to figure out how to help her newcomer students she has had in the past feel as if he was a part of the classroom. She specifically states newcomer students should be included at all times and not sent into the hallway with other work.

\section{Neutral/Positive Disposition}

Teachers who I have determined have a neutral disposition also expressed a positive attitude about working with their emergent bilingual students when asked specifically using the 
questions from Stanoscheck Youngs and Youngs, Jr. (2001). A neutral disposition categorization does not mean that teachers in this category did not have a positive attitude toward teaching and find joy in the challenges presented to them in the field. On the contrary, it appears that their positive disposition toward emergent bilingual students is a result of their generally positive disposition toward teaching itself. They did not, though, demonstrate a positive attitude specifically toward teaching emergent bilingual students. Neither did they demonstrate an understanding or desire to learn strategies unique to this population of learners. Rather, it appears that teachers in this category are quite pleased to work with emergent bilingual students, but would be equally happy to work with any child who walks in their doors and will view each of them as unique learners.

Teachers in the neutral category are Jennifer and Susan. While both Jennifer and Susan provide a very positive reaction to working with emergent bilingual students, these teachers did not indicate the benefits to both the teacher and other students that come with working with culturally diverse students. They seem to simply look at their emergent bilingual students as they do for any other students. They are simply similar to all their other students in the sense that they have a particular learning profile. As their teachers, they indicate that they are not daunted by striving to meet their students' individual needs.

Jennifer. In response to the disposition question asking about how Jennifer would feel if she were given two or three emergent bilingual students next year, she states,

I'd be okay with it; they're kids, they're kids. I mean, I've got kids at all levels and you have to make modifications to what they're capable of doing. You know, like Frederic, I would expect of him, he could at least give me three paragraphs in an essay, maybe not the five at least three. And he could give me four to five sentences. And then I have 
students that I can only take at least two sentences for each paragraph because that's all they can do. So I just kind of treat my Els like they're just students and we all just have different learning levels. (Interview 2).

In this way she describes how she makes modifications for all students. She is happy to teacher emergent bilingual students at their level because that is what she would do for all students who walk through her classroom door.

Susan. Susan's response to this same question was that she would feel, "a bit nervous, as I'd want to be able to meet each of their needs. But I'd investigate how I could best do that and rely on my resources for help." Then, in a cheerful tone, she states, "By the way, I've heard I'll have all of the EL kids for $4^{\text {th }}$ grade next year!" (Interview 2). In this statement, it's clear that she knows there are specific strategies that are helpful with this population and does indicate that she would make an effort to learn more here. This response is similar to those of teachers assigned to the positive disposition group.

Regarding her overall reaction to working with emergent bilingual students, Susan states, "I enjoy working with them-and especially seeing them blossom in confidence from the beginning of the year to the end!" (Interview 2). Again, while her responses suggest a generally positive attitude toward working with emergent bilingual students, there is nothing specifically positive about the unique abilities emergent bilingual students bring into the classroom.

\section{Neutral/Negative Disposition}

The neutral/negative disposition is similar to the neutral/positive disposition in that the teacher does not cite an enjoyment in the unique abilities presented by their emergent bilingual students. One significant difference, though, is that the neutral/negative disposition also teaches students as using a generally Ameri-centric perspective This is not to say that the teacher in this 
category does not spend time getting to know her students individually or that she is open hostile to her students. However, she does appear to consider "different" as synonymous with "bad" and therefore attempts to address and teach student as a homogenous group rather than celebrating their uniqueness.

Only Melody was categorized as neutral/negative. When asked specifically how she would feel if she were assigned to work with two or three emergent bilingual students next year, she simply said, "It would be great" (Interview 2). When asked to describe her overall reaction to working with emergent bilingual students, she said:

Years ago I was intimidated when I first heard about [a new immigrant population moving in] because I was pretty I and thinking, “Oh my gosh! The’ don't know any English how am I going to correspond? What am I going to do?" I was putting myself in their shoes and I think I was worrying more than I needed to especially at this age because at this age they're so willing to learn and they want to. (Interview 2). Neither of these responses seem particularly negative.

However, again, other responses and statements in the interviews from the participant resulted in a neutral/negative categorization. Her comments suggest that she believes that students should be expected to fully participate in the activities of the class without the teacher providing differentiation. She mentioned several times that she does not like to be singled out and therefore does not want to single out students by providing differentiated materials, instruction, or one-on-one assistance without it being offered to all the students in the classroom.

At one point, the participant shared a story in which an emergent bilingual student of hers used her classroom phone to call her parents and speak in her L1. She described her own shock and the shock of her students when listening to the child speak in another language. She said the 
student felt uncomfortable and so that teacher tried to help her "celebrate that she was fluent in two languages." Melody goes on to say, "so now she does silly stuff to me in the hall an' she'll talk to me in her native language'. It's kind of funny." Melody continues with, "I thi'k it's also nice that they get to keep that to themselves and use it when they need to and feel comfortable here as well" (Interview 2). This comment suggests that, in general, the linguistic diversity of her students is not perceived as an asset to the classroom community. Melody's comment may be aligned with deficit thinking at worst in that she would like the child to keep her native language to herself. Melody's perspective may also be seen as ineffective teaching practices at best in that she is not recognizing the linguistic resources her students bring to her classrooms. Yet Melody's statement may also be a result of a common myth of language acquisition. Namely, that one learns languages best in an immersion setting. She may have thought that students would not feel comfortable speaking their home languages and just wants students to feel comfortable around their English-speaking peers.

However, at another point in the interviews in which Melody discussed her travels abroad, she seemed to come across as mocking. In her first interview, she described a vacation to Italy in which, due to a mistake she had made in dialing, the Fire Department was called to her room. She stated, "Even when they pulled up I thought are you serious?' That's their fire department? It was literally like a red pickup truck with a garden hose. And then I was paranoid I was like what are they doing here?" (Interview 1). In this way, although the fire department had come to help her in an error she had made, she looked down on them as inadequate.

In further discussing her trip to Italy, she describes a moment while visiting the Coliseum in which she started to feel very uncomfortable: 
I don't know if I just psyched myself out but all these people are moving in and I was just not comfortable at all and I couldn't talk with them because they're speaking every language BUT English. We hooked up with a tour guide, you know, [he said] 'O' you're American.” And [I asked] "How do you know that?" You know, we didn't really say anything. And he was telling me this story about how you can always tell the Americans by their shoes and I'm like, "Shoes? What?" So we started to notice and that's probably what I learned most on that trip (Interview 1).

Here Melody mentions that her biggest takeaway from her visit to a foreign country was that Americans tend to wear different shoes than Italians or other tourists as opposed to taking away insights about the culture.

Ultimately, Melody was categorized as having a neutral/negative disposition due to three main factors. First, she was the only teacher not to provide differentiated instruction throughout the observations and stated in the interviews that this is not a teaching strategy she supports. Secondly, Melody did not understand the value of the linguistic resources her students bring to her classroom. Finally, her description of her travel experience to Italy reflected an Americentric world view.

\section{Deficit/Complex}

Determining Linda's disposition toward emergent bilingual students was more complicated. As a former emergent bilingual student herself, she describes feeling a lot of empathy for her newcomer students. Throughout the interviews, she often expounds upon her own experiences as an emergent bilingual student and mentioned that she did not want her emergent bilingual students treated the way she was treated when she was a newcomer. She 
clarifies this idea with her personal story of being a 5'7" sixth grader and sent to a second grade classroom because her teacher did not know how to teach her. She said:

When I received when Edouard, he was my first student about three to four years ago... I wasn't going to do the same thing to him as my sixth grade teacher did to me, which was leaving me alone over there in the corner and giving me a pile of books that didn't know what to do with me. And she even said she had no idea what to do with me. (Interview 1). This theme of using her experiences to shape the way she approached working with her newcomer students is presented several times in the data. This specific approach will be discussed later in the chapter.

Linda exhibits characteristics of both the positive and negative dispositions. Evidence of a positive disposition is how she describes her emergent bilingual students as a benefit to her class as whole. She describes that she is excited to see her American students understand the diversity of the world and to hear more than just English. In answer to her overall reaction of working with emergent bilingual students, she says:

My reaction? I like them. I just really think it's great. It's refreshing to... to help kids who are not... who are struggling, coming in, in a different sense, in the different way, coming in... in terms of language and see the progress. I mean you can definitely see the progress so it's great and to see that is a benefit for the other kids to see because everybody is pretty homogeneous so having him and it really helps other people in the classroom understanding empathy for him and around him because they understand how he struggles. He sees it. They see how his head is going, spinning (Interview 2). 
In this sense, Linda certainly sees the unique benefits emergent bilingual students present to the classrooms in which they are enrolled. This observation is a characteristic of the positive disposition of teachers.

However, deficit ideology is also repeatedly present in the interviews with Linda. While she states that she does not want to frustrate her emergent bilingual student with work that is beyond his reading level, she says, “He can’t do science. He can't do social studies. He can't read." Later in that same interview, she states, "he couldn't read, he couldn't do math" (Linda, Interview 1). A third time in the same interview, as she discussed her newcomer student's grades using a one to four standards-based grading system, she states, "I said he is at a 1 he's gonna be at a 1 pretty much the whole entire year and [the principal] was okay with that" (Linda, Interview 1). In comparing her work as an emergent bilingual student to the work that her student puts into learning the language or doing homework, she states:

You know, my learning experiences are very different from other people's learning experiences. My learning experience was like I was driven. I had literally 3-4 hours of work on the floor and I had to go through different dictionaries and trying to learn it. For [my newcomer student] he didn't have that support he didn't have the educational background that I had. He came he didn't know how to read. He didn't know how to do math, you know. He was different. He was just... So you know, I couldn't expect the same thing from him. (Linda, Interview 1).

While Linda acknowledges that her experience with reading and math in her home language was beneficial to her learning and was a resource that her newcomer student did not have, she also implies that he did not have the same motivation that she had. When saying her learning 
experiences were different, that she was driven, and that she was different, the implication is that her newcomer student is not driven.

These three statements very clearly fall in line with deficit ideology. Again, deficit ideology is identified by six characteristics. The first of these as listed by Valencia (1997) is "blaming the victim" while the fifth is "educability". While Linda does not explicitly "blame the victim", while comparing herself to her emergent bilingual student imply that he does not having the motivation and drive she had. She catches this and follows up with an acknowledgement that he does not have the same home support she had. She states, "The' don't have that support at home so I can judge them, I can get mad at them or I can turn it around and see what I can do in the classroom to see progress" (Interview 1). Furthermore, Linda acknowledges that her newcomer student has made some progress, which suggests that she recognizes his ability to learn, or his educability. However, in interviews, Linda states that she often sends her student out of the classroom during all the subjects she believes he "can't" do. She states that he doesn't have the background ability yet and that he would be too "frustrated".

When framed in the deficit mindset, the limitations she perceives of her students takes away the responsibility for teaching. Rather than finding a way to teach her student with the funds of knowledge he brings to the classroom, she sends him out of the room for lower-level thinking tasks, such as math drills, and asks volunteers, student teachers, and peers to be responsible for his learning.

Despite the comments Linda made that are in line with deficit ideology, she also seemed to have complex disposition toward her emergent bilingual students. She also discusses the fact that she wants other students in her class to see where her emergent bilingual students are 
coming from and to have an understanding of him in order to help accept him into the classroom community. She says,

and it also helps to let the other kids know that you're trying to help that kid so they as a class they can see it so they wouldn't be picking on that kid as, 'You need to learn English.' Because in this country, I think that's really helpful because to know that in this country it's okay to have more than one language because I told them I have about 6 under my belt. And so they're like, 'Oh my God can you do this?' so I teach them several languages counting with a different systems. (Interview 2).

Due to the complex nature of Linda's responses, I have categorized her disposition as deficit/complex. She clearly sees the benefit of having an emergent bilingual student on her American students. She also seems to understand the challenges her emergent bilingual students face. However, while she states that she wants her emergent bilingual student feel included, it appears that this only applies to his social experience as she often sends him out of the room for academic tasks.

\section{Disposition Continuum or a Multifaceted Construct}

The literature on teacher dispositions toward emergent bilingual students is often framed as a negative disposition or a positive disposition; a deficit ideology or a non-deficit ideology. The LATS instrument developed by Byrnes and Kiger (1994) creates an interval-level variable, which is to say, represents disposition on a continuum between these two binaries. For the White, monolingual, American teachers who participated in the study, this continuum could be an accurate representation of their dispositions. While this continuum may not be fully representative of their dispositions, five of the six teachers were able to be placed along this continuum. Figure 1 demonstrates what that continuum would look like. 


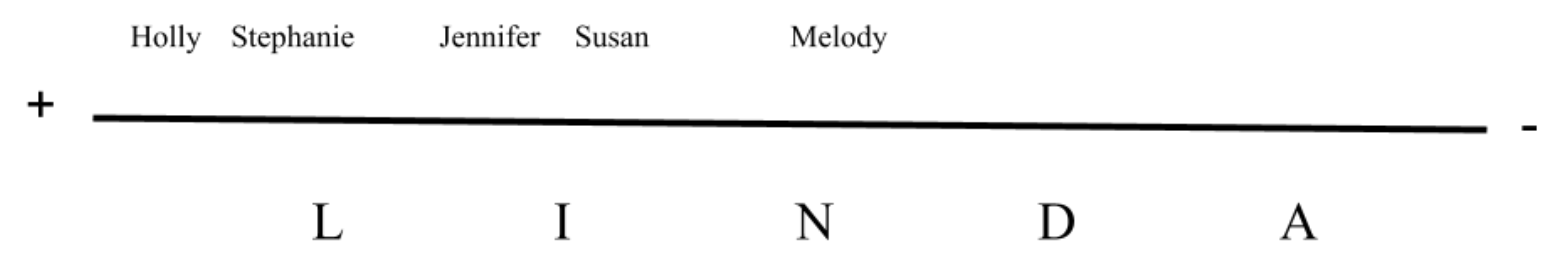

Figure 1. Participant's dispositions on a continuum.

However, what appears to be more reasonable than the continuum perspective is that teachers' dispositions are much more nuanced than this continuum suggests. Dispositions toward emergent bilingual students appear to be multifaceted. Some of these facets could be beliefs about how language is learned, beliefs about students in general, beliefs about effective pedagogy in a classroom of diverse learners (emergent bilingual or otherwise), opinions of other cultures, opinions of people from races, ethnicities, and countries different from one's own, opinions on the role of the classroom teacher vs. the ESL teacher, personal background, and views of teaching in general, to name just a few. Linda, with her polylingual and multinational background, presents a deficit-based yet at times positive complex perspective toward working with her emergent bilingual students.

Now that teacher dispositions have been discussed, I can report the research findings. I will provide evidence for each conclusion using the data from the teacher participants. Results from this study will be reported according to the cases in order of disposition from positive to deficit/complex, when possible. 


\section{Qualitative Findings for Research Question One: What Influences the Dispositions that Teachers Hold Toward their Emergent Bilingual Students?}

There were four factors that appear to influence a teacher's disposition toward their emergent bilingual students. These included personal experience with bilingual individuals who immigrated to the United States in their childhoods, outlook on teaching and students in general, international experience, and experience teaching emergent bilingual students.

\section{Personal Experiences with Bilingual Individuals who immigrated to the United States in their Childhoods}

Two of the teachers involved in the study were able closely related to their newcomer emergent bilingual students. The first teacher with a personal connection to a former emergent bilingual student experience is Holly. Holly is a fourth-grade teacher who is currently married to another fourth-grade teacher in a different school district who immigrated to the United States from Mexico when he was in fourth grade. During the interviews, Holly mentioned her interest in cultures around the world several times. She says:

How can I still, like, better meet [my emergent bilingual student's] needs and learn 'cause

I love learning about culture? It just makes me very happy and I love seeing different insights and perspectives and I would love to be able to talk with the students and get their input and stuff" (Holly, Interview 1).

She also states' "It's like I want to be able to share in that culture and have all my students realize I'm just as invested in where they came from as where they are" (Holly, Interview 1). She describes herself as "hooked on the learning about other cultures" (Holly, Interview 1).

Throughout the interviews, Holly often drew a connection between her husband's experience and that of her students. She runs ideas past her husband to see if he would have 
wanted a teacher to use certain practices with him when he was a child or if those practices would make him feel uncomfortable. In discussing how she turns to her husband's experience as a resource in her teaching, she states,

And he really suffered because he didn't have teachers who really wanted to take the time to include him. So I always try to go to him being, like, "Okay, how would you feel? How would you feel if this happened?" Because I never want to put my kids in the position where they feel left out or different or anything like that. And, so I've gone to him quite frequently, "Hey, you know, in your experience how would this have made you feel? Or how does this come off as...? Would this be embarrassing or too hard?" And he's not my kids, but he's obviously got some insights that I trust. (Holly, Interview 2). She makes decisions based on her husband's experience and feelings, but is aware that his experiences may not accurately portray the complexity and uniqueness of what her students are experiencing.

The other teacher, Linda, described family as "ethnic Chinese" living in Vietnam. She faced discrimination in Vietnam and, in her words, her family "was basically kicked out of the country." While she primarily spoke Fukienese with her family, she also learned four languages in her childhood (Vietnamese, Cantonese, Taiwanese, and Mandarin) before coming to the United States when she was in $6^{\text {th }}$ grade to learn English and French. She later moved to Japan for one and a half years and studied Japanese. In describing her English language learning experience, she states, "I was in a middle school with a whole entire ESL department and so I learned through ESL" (Linda, Interview 1). Therefore, Linda has learned and acquired languages using several different methods, which may be similar to what her students are experiencing. 
Throughout the interviews, Linda repeatedly related the experiences of her students to her own experiences as an emergent bilingual student. She mentioned that she was determined not to treat her emergent bilingual students like she was treated. She states:

Many of it has to do with the fact that I came from a background where I, you know, my sixth-grade teacher left me alone in the corner. She actually sent me to a second-grade class thinking that maybe I was I would be suited in second grade, you know. So I was like a 5'7"' big girl going to second grade with little munchkins for a whole week and the kids at the beginning thought, "Oh my god we have an aide in here" you know. And when they realized I couldn't speak to them they started leaving, like skedaddle, you know, like literally like scattered kids. And so my experience has helped me become a better teacher to become a better um uh strategist for [my newcomer student], for all the students who come into my classroom and have more empathy for them to see that they are struggling and to be able to see that look (Linda, Interview 1).

Linda's experience was closely tied to her own educational decisions. As mentioned previously, she compared the way she went about learning English to the way her newcomer student studies. She concludes by stating that as a result of this difference, she held lower expectations for his work. In this way, by comparing of her personal experience to what she observes from her students she determines what she believes she can or cannot expect her students to do.

Both of these teachers are making an assumption, to some extent, that their students will feel and experience school the same as they did. It fails to recognize that these students may not feel the same. Their students are different people, from a different culture, in a different time period, who may speak a different language. However, their personal connection to emergent bilingual students is a resource that they are uniquely able to call upon. This resource can be used 
to gather information on what they may consider to be best practices, regardless of whether or not they are, and may also be used to set expectations for what a child should or should not be able to do.

\section{Outlook on Teaching and Students in General}

Jennifer and Susan were both identified as having neutral dispositions toward their emergent bilingual students. These teachers spoke about their emergent bilingual students as if they were just like other students in their classrooms who have a unique learning profile. They discussed the way they fit their emergent bilingual students into their differentiated instruction. This included pulling them into specific small groups for additional assistance in a reading and writing task (Jennifer) or working them into existing reading groups (Susan). Both teachers included their emergent bilingual students in their whole class instruction as well. These teachers recognized that the language difference would necessitate some specific teaching strategies.

During their interviews they expressed that all of their students had specific needs and they have an obligation to consider these needs as they taught. Jennifer stated, "I just kind of treat my Els like they're just students and we all just have different learning levels" (Jennifer, Interview 2). Susan approached teaching her emergent bilingual students in a similar manner, and talked about getting to know the child, assessing needs, and differentiating according to those needs.

\section{International Experience}

The teacher participants in this study demonstrated a variety of travel experience. The table below depicts the international experience of each participant. 
Table 4

Teacher Travel and Disposition

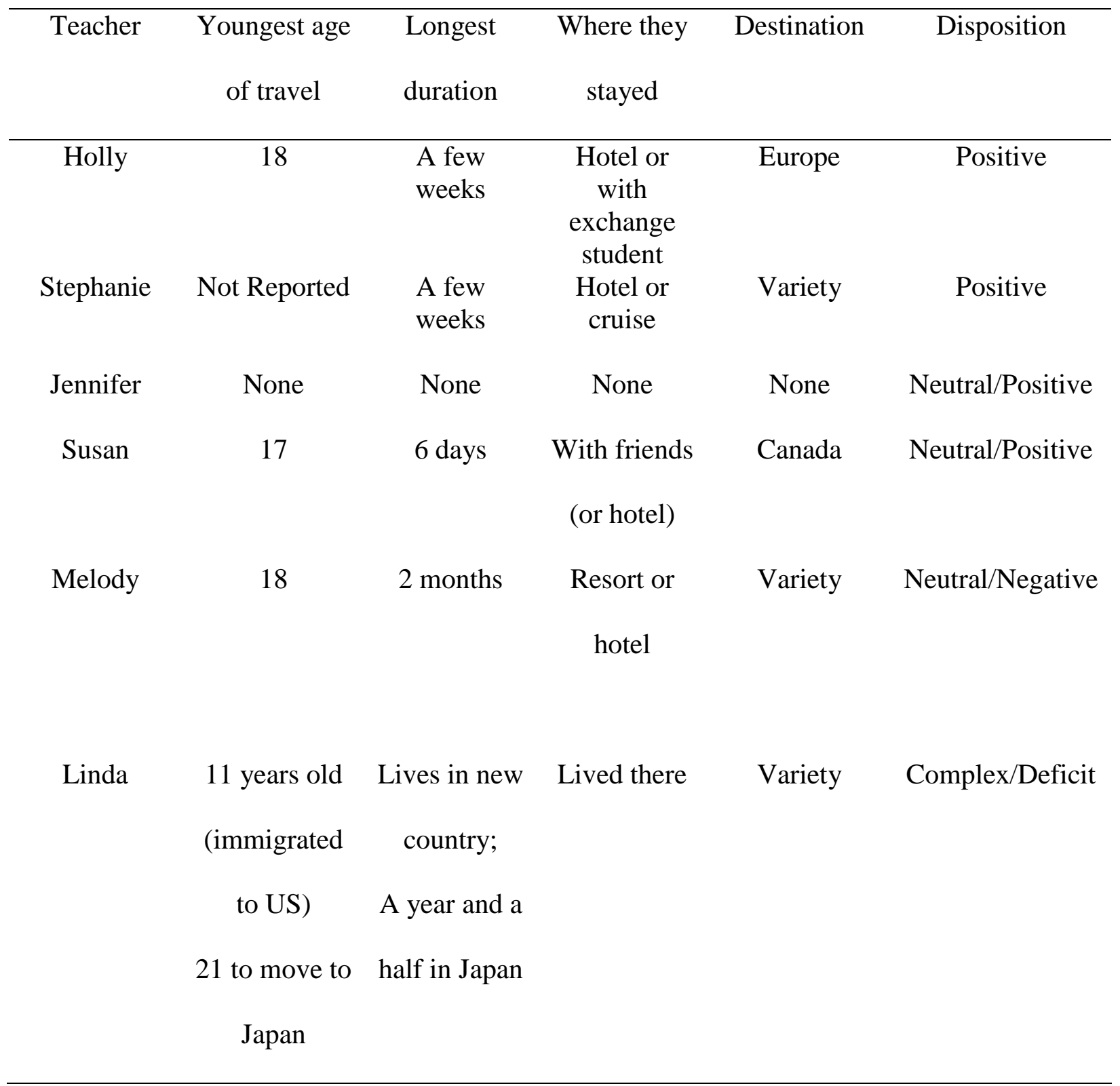

As you can see, the participating teachers had a variety of intercultural experiences in all areas. These participating teachers' experiences do not support the idea that the greater amount of travel a teacher has, the more positive his or her dispositions toward emergent bilingual students. Teachers represented a variety of dispositions regardless of where they traveled, the duration of 
their visits, where they stayed when they traveled and how old they were when they first traveled out of the United States.

Holly, who was identified as having a positive disposition toward emergent bilingual students, discusses her experience of hosting a foreign exchange student as well as dating and then marrying her current husband whose family emigrated from Mexico. These experiences shaped her outlook on different cultures. She states:

That was my first experience of having somebody with a different culture in my personal space because we're sharing a bedroom. But it was, like, really cool to hear about what, like, even though, like I said she came from a country where most of them do speak English she like she just had so many different life experiences than me. Even though we were at the same age, we actually kind of even looked alike, our worlds had been completely different growing up. And learning about that education process of another country, which really interested me because they do everything's so different than the United States. And just, like, traditions with your family... I didn't realize it was really important for them to all live like almost like in a commune with people they don't even know. But the feeling of family and belonging, whereas you know, we try to keep our apartments as far apart as possible they want it as close as possible. And that was just really cool to me. And then I actually got to go over to Denmark to visit her a couple years after she left and I stayed there for 3 weeks and it was really cool to see everything that she had told me, like to see it firsthand for myself, like to experience it. So that's what really got me hooked on the learning about other cultures because here was this girl that look like me that kind of sounded like me and I didn't think you were that different and then like seeing how 
different we actually were. And so I think a lot of that even kind of went into when I started dating my husband. Like he comes from a very proud Mexican family and they, my husband and his siblings, speak English but his parents do not and I really had to find ways to communicate because I wanted to find ways to be a part of their family. I wanted to impress them and wanted to marry their son. But, like, just like seeing how they celebrate different holidays. Like, I thought Thanksgiving was big for everybody. Doesn't make sense to celebrate Thanksgiving if you're from Mexico because it really doesn't pertain to you so there's different things that I didn't really think about. But just really experiencing different traditions that's what I'm mostly interested in. Well, I love food from everywhere. I'm a big foodie. But, like, I really like experiencing the different ways families from different cultures come together and how they celebrate that together time. Like, that just really interest me. (Holly, Interview 1).

Although Holly had only traveled to a foreign country for a few weeks, her experience of hosting a foreign exchange student from Denmark and her interaction with her husband's family were intercultural experiences she had in the United States that made her want to learn more about people around the world.

Like Holly, Stephanie has also both had international travel experience and maintains a positive disposition toward her emergent bilingual students. Stephanie has traveled to Ireland, Paris, Hawaii, the Bahamas, and other places. She stated that "it's scary" to travel somewhere and not understand the language. She mentioned that she feels that it helps her understand where her students are coming from. 
Jennifer, on the other hand, has not traveled outside the United States but uses teaching practices that recognize the unique needs of her students. In the interviews she mentioned that she would like to use books to help her native English-speaking students learn Spanish while her emergent bilingual students learn French. Despite her lack of travel experience, she sees the value in learning a foreign language and having all her students learning languages together. This is evidence of her respect for the languages and cultures of her students.

Melody, by contrast, has traveled more than many of the other participants. However, she maintains a disposition more rooted in deficit ideology or language acquisition mythology. Melody has been to Italy, Switzerland, Mexico, and Jamaica. However, as mentioned earlier, in discussing her travels she mocked the Italian fire department and mentions that the biggest thing she learned was how to identify Americans by their shoes.

Therefore, it appears that international experiences can be transformative with the power to disrupt deficit beliefs about cultures and countries outside of the United States, but not at all times. The quality of the experience plays a larger role in transforming deficit mindsets than the duration, distance traveled or frequency. The participants who described a transformative experience also mentioned strong and close relationships. This concept may need to be further investigated to determine what aspects of international travel are particularly useful in transforming deficit ideology.

\section{Experience Teaching Emergent Bilingual Students}

Case study participants represented various levels of experience in working with their emergent bilingual students. As depicted in Table 5, Holly, with the least amount of teaching experience, had the most of experience working with emergent bilingual students, in terms of the number of emergent bilingual students she has taught. This is the case because for one year she 
served as a library teacher in a school with a high number emergent bilingual students. As she states, she taught every emergent bilingual student that "walks through the doors" of the school. Table 5

Teacher and Experience

\begin{tabular}{lcc}
\hline Teacher's Name & Years Teaching & Number of Emergent Bilingual Students \\
\hline Holly & 3 & $40+$ \\
Stephanie & 22 & 4 \\
Jennifer & 11 & 5 \\
Susan & 39 & 3 \\
Melody & 19 & 12 \\
Linda & 22 & 3 \\
\hline
\end{tabular}

Many teachers described feeling nervous about working with emergent bilingual students. Some recognized changes in their thinking as they gained experience teaching emergent bilingual students. In describing how they felt when they anticipated working with this population, teachers stated they felt "nervous" (Holly, Interview 1), "lost” (Holly, Interview 1), “panic” (Holly, Interview 1), “apprehensive” (Holly, Interview 2), “overwhelmed,” (Stephanie Interview ), "flabbergasted” (Susan, Interview ), “petrified” (Melody, Interview 1), “worried” (Melody, Interview 1), and describe the experience as "nerve-wracking" (Holly, Interview 1), felt as if they were "floundering" (Holly, Interview 1) and "thought it was going to be horrible" (Melody, Interview 1). Stephanie describes her reaction to working with students as "scary at times when they come in and they're overwhelmed and they're scared and it hurts my heart because I don’t know how to help them sometimes” (Stephanie, Interview 2). 
However, some teachers acknowledge that they still have progress to make before they feel confident. When asked how she feels about working with emergent bilingual students at a variety of proficiency levels next year Holly states she feels, "Scared but excited. This is a very real reality for me next year and I am apprehensive because I don't want to fail them, but I am very excited to be their leader on their fourth-grade journey and learn things from them along the way as well" (Holly, Interview 2). Other teachers state that with more years of experience teaching emergent bilingual students, they felt more confident. Stephanie states "my experience has been very positive and I think I've shared that last time some of it has been very eye-opening to me rethink how I teach rethink that where I stand, rethink how I say things. It's been so good for my other kids too" (Stephanie, Interview 2). Melody says, "That first year as much as I thought it was going to be horrible it was great" (Melody, Interview 1). These statements indicate that while some teachers feel more confident in their current teaching practices after more years of experience with emergent bilingual students, others feel more confident because their they have reassessed and improved their general teaching practices.

Holly, the teacher with the fewest years of teaching experience is the most nervous about successfully teaching her emergent bilingual students. However, she also acknowledges that she has more to learn. Teachers who have more teaching experience (Susan, Stephanie, and Melody) also describe feeling nervous at first. After some experience, though, they felt more confident with their pedagogical choices.

Additionally, as teachers gained experience, they discovered some initial assumptions were misguided or they identified previously held beliefs that were untrue and needed to be confronted in order to better serve their student's needs. (Holly and Stephanie). Holly, again the teacher with has the most amount of experience with emergent bilingual students in the fewest 
number of years teaching, describes an area of her personal assumptions she felt she needed to reconsider after having some experience. She says

I know not all of our students that are coming over are necessarily speaking French and I was really guilty of having the... the... idea that, "Oh, they're coming from this place there that's a French-speaking place they must all speak French.” And so I think I was kind of in a panic moment of like "Wait, what do you mean they don't all speak French? How am I supposed to connect with them?" Because it was hard to find Lingala and stuff on apps like it wasn't always readily available. So I like I think that's been my biggest challenge so far is one getting past the mindset of... getting past the mindset that they're only speaking French and they're coming over and like two how can I still like better meet their needs and learn because I love learning. (Holly, Interview 1).

In this statement, Holly reveals that her experience helped her address some preconceived notions and expectations she held toward her students.

Teachers with experience working with emergent bilingual students can build on their experiences when working with new emergent bilingual students. It becomes a personal resource they can call upon later in their careers. Holly, discovered how she was able to build rapport with a Spanish-speaking student when she read Esperanza Rising or by speaking Spanish with him at school. Stephanie, who has moderate experience with emergent bilingual students and 22 years of teaching experience describes how she reached out to others she perceived as more knowledgeable at first. These included a classroom volunteer, Christina, and a former multilingual district ESL teacher and a current district family coordinator. Stephanie says, I think my first year I reached out first not knowing, not having other things at my fingertips. So I think I reached out to Christina. I reached out to David first my first year. 
"What do I do? How do I do this?" Or [I reached out to] other teachers who had had experience, now I have I have a shelf full of supplies that I can go to depending upon their needs um flashcards and games and some books and those kinds of things. (Stephanie, Interview 1).

Although Stephanie is referring to the tangible resources she has, her description of how working with her emergent bilingual students has been eye-opening and how it helped her to reconsider her teaching demonstrates that she now has some internal resources as well now that she no longer looks to an external source.

\section{Quantitative Results for Research Question One: What Influences the Dispositions that Teachers Hold Toward their Emergent Bilingual Students?}

This research question is accompanied by seven sub-questions that have been analyzed quantitatively. These questions will be considered one at a time and discussed below before providing a general conclusion. Before we can look at the sub-questions, though, as with the qualitative analysis, it is important to look at how teacher dispositions are measured. For this study, I administered the LATS 13 item survey (Byrnes \& Kiger, 1994) to teachers. Teachers were asked to rank the level that they agree with 13 statements related to "language politics, LEP intolerance, and language support" (Byrnes \& Kiger, 1994) on a Likert scale. The sum of these responses are the LATS score assigned to each participant. The score has the potential to range from 13 to 65. Descriptive statistics of the survey results for the LATS study demonstrate that the mean for the teachers of this district was 27.52 and the median was 28 with a standard deviation of 6.99 . The minimum score was 14 with a maximum score of 54 making a range of 40. The wide range and large standard deviation indicate that there is a great variety of dispositions toward emergent bilingual students that was analyzed. 
Table 6

Language Attitudes of Teachers Scale

\begin{tabular}{ll} 
Mean & 27.52 \\
Median & 28 \\
Standard Deviation & 6.99 \\
Minimum Score & 14 \\
Maximum Score & 40 \\
\hline
\end{tabular}

\section{Sub-Question One: Bilingual Status}

Do the dispositions of teachers who speak a language other than English differ from the dispositions of teachers who do not speak a language other than English? The null hypothesis that will be tested states that there is no relationship between dispositions of teachers and whether or not the teachers speak a language other than English. A one-way analysis of variance (ANOVA) was conducted to evaluate the relationship between a teacher's disposition toward his or her emergent bilingual student and his or her personal bilingual status. The independent variable - the teacher's bilingual status — included three levels (the teacher speaks a language other than English not at all, somewhat, or fluently). The dependent variable was the teacher's disposition toward emergent bilingual students as measured by the LATS score. The ANOVA was significant, $\mathrm{F}(2,55)=4.42, \mathrm{p}=.017$. Post hoc comparisons (Bonferroni) found significant differences in the means between the teachers who somewhat speak a foreign language and those who do not speak a foreign language at all. The eta-squared effect size value $(\mathrm{n} 2=.139)$ suggests a weak practical significance. The null hypothesis has been rejected at a $95 \%$ confidence level. 
From this test, it appears that even without reaching fluency, studying a foreign language to some extent is linked to a more positive disposition toward emergent bilingual students.

\section{Sub-Question Two: Travel}

Do the dispositions of teachers who have visited a country that speaks a language other than English differ from the dispositions of teachers who have not visited a country that speaks a language other than English? $\mathrm{H}_{0}$ : There is no relationship between dispositions of teachers and whether or not the teachers have visited a country that speaks a language other than English. An independent samples $t$-test was conducted to evaluate the difference in the LATS group means for teachers who have visited a country that speaks a language other than English (group 1) and teachers who have not (group 2). There were no significant differences between teachers who have traveled to a country that speaks a language other than English $(M=27.25, S D=6.52)$ and those who have not $(M=27.95, S D=7.85), t(56)=-.369, p=.71$. The $t$ value of -.369 is not significant as the $p$ value of .71 is $>.05$. These results suggest that whether or not a teacher has visited a country that does not speak English plays no role in his or her disposition toward emergent bilingual students as measured by the LATS score. Based on this information, we can accept the null hypothesis that there is no relationship between dispositions of teachers and whether or not the teachers have visited a country that speaks a language other than English.

\section{Sub-Question Three: Duration of Travel}

Do the dispositions of teachers who have spent a longer amount of time in a country that speaks a language other than English differ from the dispositions of teachers who have visited a country that speaks a language other than English for a shorter amount of time? $\mathrm{H}_{0}$ : There is no relationship between dispositions of teachers and the length time a teacher has spent in a country that does not speak English. For this test, there were 29 respondents who had spent less than one 
month in a country that does not speak English. Three respondents spent two to five months in a country that speaks a language other than English; two who spent six to 12 months and two who spent more than 12 months in a country that speaks a language other than English.

A one-way analysis of variance (ANOVA) was conducted to evaluate the relationship between a teacher's disposition toward his or her emergent bilingual student and the amount of time he or she has spent in a country that speaks a language other than English. The independent variable - the amount of time spent in a country that speaks a language other than English — included levels (less than one month, 2-5 months, 6-12 months, and more than 12 months). The dependent variable was the teacher's disposition toward emergent bilingual students as measured by the LATS score. The ANOVA was not significant, $F_{(3,32)}=3.07, p=.042$. Post hoc comparisons (Bonferroni) found insignificant differences in the means between the group who has spent less than 1 month, 2-5 months, 6-12 months and more than 12 months in a country that speaks a language other than English. The eta-squared effect size value $\left(n^{2}=.223\right)$ suggests a weak practical significance. The null hypothesis has been rejected at a $95 \%$ confidence level. The more time a teacher spends in a country that speaks a language other than English, the more positive his or her disposition is to emergent bilingual students. However, there was a slight rise in the LATS score, indicating a less positive disposition toward emergent bilingual students for teachers who have spent more than 12 months in a country that speaks a language other than English.

\section{Sub-Question Four: Length of Time Teaching}

Do the dispositions of teachers who have spent a longer amount of time teaching differ from the dispositions of teachers who have been teaching for a shorter amount of time? $\mathrm{H}_{0}$ : There is no relationship between dispositions of teachers and the length of time a teacher has 
spent teaching in general. A one-way analysis of variance (ANOVA) was conducted to evaluate the relationship between a teacher's disposition toward his or her emergent bilingual student and the amount of years he or she has taught. The independent variable — number of years he or she has taught-included five levels (1-5 years, 6-10 years, 11-15 years, 16-20 years, and more than 20 years). The dependent variable was the teacher's disposition toward emergent bilingual students as measured by the LATS score. The ANOVA was significant, $F_{(3,53)}=1.19, p=.327$. Post hoc comparisons (Bonferroni) found insignificant differences in the means between the teachers who taught for 1-5 years, 6-10 years, 11-15 years, 16-20 years, and more than 20 years. The data demonstrate that we must accept the null hypothesis that the amount of years that a teacher has taught has no significant relationship with the teacher's disposition toward emergent bilingual students.

\section{Sub-Question Five: Teaching Time Within District}

Do the dispositions of teachers who have spent a longer amount of time teaching within the school district at the center of this study differ from the dispositions of teachers who have been teaching for a shorter amount of time within the study school district?

$\mathrm{H}_{0}$ : There is no relationship between dispositions of teachers and the length of time a teacher has spent teaching in the study school district. A one-way analysis of variance (ANOVA) was

conducted to evaluate the relationship between a teacher's disposition toward his or her emergent bilingual student and the amount of years a teacher has taught in the district at the center of this study. The independent variable — a teacher's experience within this school district—included five levels (1-5 years, 6-10 years, 11-15 years, 16-20 years, and more than 20 years). The dependent variable was the teacher's disposition toward emergent bilingual students as measured by the LATS score. The ANOVA was not significant, $F_{(3,53)}=.709, p=.590$. Based on the data 
presented above, the null hypothesis must be accepted. The number of years the teacher has taught in the target school district has no bearing on the teacher's disposition toward emergent bilingual students.

\section{Sub-Question Six: Training for Teaching Emergent Bilingual Students}

Do the dispositions of teachers who have had more training in teaching emergent bilingual students differ from the disposition of teachers who have had less training in teaching emergent bilingual students? $\mathrm{H}_{0}$ : There is no relationship between dispositions of teachers and the amount of training an educator has had in working with emergent bilingual students. A series of $t$-tests was conducted to evaluate if any one particular training had a relationship with the LATS score. None of these tests were determined to be significant. The number of trainings a teacher reported to have had was then added together. An ANOVA was conducted to evaluate the relationship between a teacher's disposition toward his or her emergent bilingual student the number of training types a teacher reports to have received for working with emergent bilingual

students. The independent variable - the teacher's ESL training —included five levels as teachers could rank if they have had no training, one type of training, all the way up to five different types of trainings. Teachers were given the option to report if they have had an in-service training, a college/graduate school class, attended a conference or workshop or another type of training that was not listed in the survey. Only one teacher reported to have had all four types of trainings on working with emergent bilingual students. $57.6 \%$ of teacher report to only have one type of training and $20.7 \%$ report having only two types of trainings. 
Table 7

Teacher LATS and Emergent Bilingual Training Type

\begin{tabular}{lcc}
\hline Type & Frequency & Percent \\
\hline In-Service & 47 & $79.7 \%$ \\
College/Graduate Class & 22 & $37.9 \%$ \\
Conference or Workshop & 12 & $20.7 \%$ \\
Other & 2 & $3.4 \%$ \\
\hline
\end{tabular}

The dependent variable was the teacher's disposition toward emergent bilingual students as measured by the LATS score. The ANOVA was significant, $F_{(3,53)}=5.90, p=.001$. Post hoc comparisons (Bonferroni) was not completed for the ANOVA as one group (the group of teachers reporting 4 different types of trainings) had only one case. The eta-squared effect size value $\left(\mathrm{n}^{2}=.31\right)$ suggests a weak to moderate practical significance. Based on this data, we can reject the null hypothesis and conclude that the amount of training a teacher has in working with emergent bilingual students has a significant relationship with his or her disposition toward that population of learners.

\section{Sub-Question Seven: Experience with Emergent Bilingual Students}

Do the dispositions of teachers who have had more experience teaching emergent bilingual students differ from the disposition of teachers who have had less experience teaching emergent bilingual students? $\mathrm{H}_{0}$ : There is no relationship between dispositions of teachers toward emergent bilingual students and the amount of experience a teacher has had in teaching emergent bilingual students. A one-way analysis of variance (ANOVA) was conducted to evaluate the relationship between a teacher's disposition toward his or her emergent bilingual 
student and experience teaching emergent bilingual students as measured by the number of students they have worked with. The independent variable - the number of emergent bilingual students the teacher has worked with - included five categories (five or fewer, six to 10, 11-15, 16-20, 20 or more). The dependent variable was the teacher's disposition toward emergent bilingual students as measured by the LATS score. The ANOVA was significant, $F_{(3,53)}=2.92, p$ $=.03$. Post hoc comparisons (Bonferroni) found significant differences in the means between the group who worked with five or fewer emergent bilingual students and those who worked with 20

or more. The eta-squared effect size value $\left(n^{2}=.18\right)$ suggests a weak practical significance. Given the data presented above, the null hypothesis must be rejected. There is a relationship between the number of emergent bilingual students a teacher works with and his or her disposition toward that population.

\section{Qualitative Findings for Research Question Two: What Resources and Strategies Do}

\section{Teachers Draw Upon in Working with their Emergent Bilingual Students?}

Both the qualitative and quantitative results for the research questions will be presented in two sections. First, I will provide a discussion of resource use and this will be followed by a discussion on strategies. Following the discussion of quantitative results, I will provide the conclusions that I drew from combining the data.

\section{Resources}

Cohen, et al. (2003) create a distinction in the general term "resources" to describe a difference between personal, environmental, and traditional resources. The data suggests that these resources can be further broken down into resources teachers use for instructional purposes (instructional resources) and resources teachers use to learn more about pedagogy (pedagogical resources). For example, a teacher may use a volunteer in her classroom. That volunteer may be 
someone who always works with the third grade in that building filling whatever needs the teachers have. This is an example of an environmental resources as this volunteer is associated with that particular building. One teacher may use the volunteer to work with the emergent bilingual student providing reading instruction. Because this volunteer is actively working with the student, this is an instructional resource. However, the teacher across the hall may use this same volunteer to ask questions about reading with emergent bilingual students, utilize resources that this volunteer brings with her, and utilize the volunteer in more of a mentorship role. In this situation, the volunteer becomes a pedagogical resource as she is providing the teacher with tools to improve that individual teacher's pedagogy. With this distinction in mind, I coded the qualitative data in terms of personal, environmental, and traditional resources and further separated these into instructional or pedagogical resources.

Resources for emergent bilingual pedagogy. There are three areas that participants drew from as they sought to learn more about how to teach their emergent bilingual students. These are the resources I have called "emergent bilingual pedagogy." Teachers drew from their own teaching experience, from people they sought out to help them, and from their own empathy. These will each be described in more detail below.

Teaching experience. Regardless of their experience working with emergent bilingual students, teachers who had been teaching in general for a longer amount of time, tended to depend more upon their personal resources and less on traditional resources. Susan (39 years teaching) and Linda (22 years teaching) reported depending upon "common sense" (Linda, Interview 1), "motherly love" (Linda, Interview 1), "intuition” (Linda, Interview 1) or reading "body language" (Linda, Interview 2) or a "look on a [a student's] face" (Susan, Interview 1) to assess comprehension. As previously mentioned, Stephanie has stated that with more teaching 
experience she looks inward as opposed to looking outside of herself when she was a younger teacher with less experience. These can each be considered personal resources that they developed over time working in the teaching profession. Susan mentions that she would look at the online help kit created by district ESL teachers (an environmental resource), if she had more time. Linda, by contrast, seems to feel almost a disdain for traditional resources, stating that she uses books as resource least of all of the resources (Linda, Interview 1). She also says, "a lot of it is basically looking at the student and seeing what they need. And it's not scripted in a book" (Linda, Interview 1). The newer teachers, Holly, Jennifer, and Melody made no mention of these internal, instinctual resources.

With less teaching experience, teachers looked primarily to others for help answering questions on working with emergent bilingual students. Melody (19 years teaching) said she would occasionally send an email to an ESL teacher in another building with a question. Jennifer (11 years teaching) said she would often turn to a fellow teacher. Holly (3 years of teaching) describes using a variety of people to get ideas for working with her students including the school ESL teachers and her husband who she described as "my little PD man" (Holly, Interview 1).

Holly by far had the greatest experience working with emergent bilingual students and, at three years, had the least amount of teaching experience in general. She, however, reported that she used the greatest variety in the resources she turned to for pedagogical questions. She reports turning to her husband (personal), the school ESL teacher (environmental), grade level teachers around the district (environmental) and the internet (conventional). She has also expressed an interest in a desire to learn more and take more PD (conventional). 
Self-selected resource person. As previously mentioned, all teachers expressed apprehension when they were first assigned to work with emergent bilingual students. When experience this trepidation, four participants identified turning to someone to help them teach this population. For three of these teachers (Holly, Stephanie, and Jennifer), I have decided to label these individuals as mentors. For these teachers, the individuals to whom they reached out provided guidance and suggestions for the teachers and offered ideas to use in their classrooms. These mentors taught the teachers what the teachers perceived to be effective strategies for emergent bilingual students. Melody mentioned that she occasionally emails another teacher for sharing ideas, but this is not as intense, direct, and had a shorter duration and frequency than the people Holly, Stephanie, and Jennifer mention as a resource. Linda did reach out to a volunteer. However, this volunteer, served more as an instructional resource than as a pedagogical resource as Linda used the volunteer primarily to work with her student and not as much to teach her what she can do for her student. Susan did not report turning to a mentor.

In selecting a person as a primary resource, teachers chose first a resource person who had two characteristics. The first is that the teacher and resource person have a friendly relationship before they have been selected. The friendly relationship appears to be the first qualification. Rather than seeking help from the building ESL teacher who has a specialized endorsement or degree in this area, teachers would find someone with whom they had a relationship. For this reason, the resource person has been coded as a personal resource. The second characteristic is that the resource person has experience the teacher perceives as useful for the emergent bilingual student (e.g. a reading specialist, a teacher with grade level experience, ESL experience, or language teaching experience). Table 8 shows who the teachers 
identified as their resource person, their relationship with that individual, and the specialized knowledge that identified individual possesses.

Table 8

Teachers and Resource Persons

\begin{tabular}{lcl}
\hline Teacher's Name & Relationship & Resource Person Attributes \\
\hline Holly & Husband & Experience as an emergent bilingual student and also a \\
& & $4^{\text {th }}$ grade teacher \\
Stephanie & Former co-worker & Experience teaching ESL and foreign language teacher \\
Jennifer & Friend & Teacher of the same grade in a different building \\
Susan & No resource person & No resource person \\
Melody & No resource person & No resource person \\
Linda & Neighbor & Retired reading specialist
\end{tabular}

Empathy. Empathy, a personal resource, was reported by several teachers as a resource for pedagogy. Many described imagining what the students feel like and they design their instruction based on what they perceive this to be. Melody states that she routinely begins her school year by having individual conversations with her students to get to know them. She states:

I thought to myself, "How would it feel in their shoes?" I thought, "Oh my gosh. They're coming to a new country..." and I knew that they were brand new to the community because of my real estate background my family helps them with housing. So I thought, "They're coming to a whole new country, to a whole new home, a whole new school. Everything is different. What am I going to do? What if they break down on me?" I was more worried about the emotional part of it for the student and I think that really forced 
me to focus on them more than anybody else at that time just so they're comfortable here at the school. I didn't want them to give up on school or to feel like an outsider. (Melody, Interview 1).

For this reason, Melody spent most of her first month with her first emergent bilingual students learning about them. She later mentions that she does not change her teaching to accommodate her emergent bilingual students, however, she uses her empathy to learn who they are.

Linda also spoke at length about empathy. As mentioned earlier, as a former emergent bilingual student herself, she empathizes with her current emergent bilingual students. She talks about how she explicitly teaches community within her classroom to all students. She states: Most of these kids really have learned to have patience because in the classroom we talk about community. We talk about learning as a community, We talk about how do you talk to somebody without having to insult them. What language? What verbage? How do you, you know, turn your conversation around? Instead of saying, "I want that." I'm talking" how do you turn around so that you are you are giving other people a chance to speak (Linda, Interview 1).

Linda talks about how her American students have taken in her emergent bilingual student. She states, "They have taken him in as like the best buddy. They all have help him out. The girls and the boys" (Linda, Interview 1). Therefore, to Linda, her empathy means that she teaches all her students in her classroom to create a classroom community in which all are welcomed and valued.

However, Linda also allows empathy to permit lower expectations of her emergent bilingual students. When describing a conversation she had with her principal about the academic challenges her newcomer emergent bilingual student faces, she states 
I said he is at a 1. He's gonna be at a 1 pretty much the whole entire year. And she was okay with that, you know. So instead of instead of expecting him to be like everybody else she is having her empathy.

In this way, Linda uses empathy she has for the challenges her student faces and the limited home support he receives as an excuse to lower expectations for him.

Like Linda, Stephanie also describes using empathy to determine the curriculum to which her students are exposed. In Stephanie's case, she discusses using empathy to help her achieve balance in work that she assigns her EBB students. She states,

I found it very helpful that there were things they could do then we were working on other things that were too hard, uh reading level wise. So they could practice their spelling words, they could write spelling words in sentences and that. The first-grade curriculum had enough with pictures and things that they were able to do it on their own. I never wanted have them feel left out but yet as soon as I would see tears it would break my heart and I wouldn't want them to you know, feel that way. I remember last year Gloria, it was unbelievable how quickly she caught on. She became well-spoken very quickly, quicker than Jean or um I can't remember the other little boy I had last year. Um, so I thought things were going well, and I was so proud of her and I was praising her and all these things. And then we had a conference, a parent teacher conference. And come to find out she was going home from me and crying because she didn't feel like she was doing well. She didn't feel like she was keeping up with the kids. So that that's really hard to know how far to push them, but yet make them feel comfortable (Stephanie, Interview 1). 
In this way, empathy was a resource Stephanie used to determine how hard and to what degree she could provide her students with a rigorous

Instructional resources. When asked to consider the resources that teachers turn to for help teaching their emergent bilingual students, teachers who have been in the profession longer often described the instructional resources that are used help teach their students, while newer teachers describe resources they turn to in order to help them learn how to teach the emergent bilingual population. These more veteran teachers describe tools such as technology for finding content in the home language, Google translate for translating the lunch menu options, apps for communicating with parents in their home languages, websites for reading and vocabulary instruction, and other such tools. These are traditional resources, but because they are used with students, they fit my definition as instructional resources and I have coded these as strategies. Linda and Stephanie also sought help outside of the school to have community members come in and volunteer in their classrooms with their emergent bilingual students. These individuals can be considered a personal resource for instruction. For Stephanie, the volunteer that she found had specific training in how languages are learned and taught adult ESL classes. This individual also served a mentorship role for her as described above. Linda uses a number of volunteers for helping all of her students in reading and math. The volunteer she talked about several times is a retired reading specialist. While this volunteer had the possibility to act as a mentor to the teacher, Linda said that she would leave these strategies to her. She states, Ms. Amy gave me a list of you know vocabulary and words to work with the phonics to work with, you know. I don't depend on those as much as I'd let her do the work herself now. You know so when he comes to me when I read something, if he doesn't get it, I 
will break it into chunks for him but he has those things that are in place from Ms. Amy who has helped him with it.

In this sense, Linda delegated the instruction of her student to a volunteer rather than learning from her to improve her instruction when her volunteer is not available. Therefore, for Linda, this volunteer is an instructional resource and not a pedagogical resource.

Resources reported to be lacking: School district money and teacher time. Teachers who worked in schools that qualify for free lunches for students (Holly, Stephanie, and Susan) all reported that one resource they do not have is time. During observations, these teachers spent time addressing behavior issues (Holly, Observation 1 and 2; Susan, Observation 1 and 2), giving hugs to crying children, helping students talk out their feelings, and acting as a mediator for two students who were in a disagreement. These needs of the students delayed the start of class and took up the teacher's time during moments of the lesson reserved for student independent practice after instruction was given.

In asking Stephanie what are the main things that takes her time away, she reports Right now social emotional [issues]. Some years it's been behavioral. This year is not terrible. Early in the year I do a lot of re-teaching and re-teaching and re-teaching and pre-teaching and pre-teaching and pre-teaching the expectations and that takes a long time especially early on. But the social emotional... right now, the things they bring to school. I have a hard time just getting to what we need to get to because of it. Just last week I had two different kids talk to me about their dad being in jail. They were very emotional about it. One was very flippant about it, like, "Hey did you know...?" The other was very distraught and it ruined his day. I've had two different girls this year who 
witnessed physical abuse in the home. One was Dad and Mom and one was boyfriend and Mom. (Stephanie, Interview 2).

Stephanie goes on to point out that the behavior and emotional issues that she addresses with her students is exacerbated by the fact that the building in which she works now has only three paraprofessionals to share with all grade levels. This is a change from previous years in which there were three within the second and third grade hallway.

In comparing her experience with the behaviors to the more affluent school that she worked in her first year of teaching, the school where Linda currently works, Holly says, It's a lot different. And, I think that the number one thing that I've taken away from it. My kids are coming in, and I'm just speaking specifically of this class, but something that's been very big this year is I have a lot of students (and by a lot I mean at least 12) of my kids that are coming in and their top priority that day is not school because something went on before school. And so trying to get them to the middle ground of just trust me and do this activity that we're doing you don't need to fight everything that we're doing. Just trust me that it's going to be fun and that you're doing it for a reason because you need to learn something from it. They're not always willing to do that and a lot of that is because they're still trying to transition to, “Okay I'm in my classroom. Mrs. Rodriguez cares. I don't need to fight her I just need to do what she's saying." It's not like I'm sitting there like, "You need to do this, this, and this." But it takes them about half a day to warm up again that's definitely not something I experienced at the other school. I did definitely have kids talking back to me and being disrespectful, but that's because they were feeling very entitled. 'Well, I didn’t pick out this activity, or my parents didn't pick out this activity so I don't want to do this activity." Whereas a lot of the frustrations I'm 
getting in here are, “This activity doesn't seem important to me right now because I'm thinking about what I have to do later" or "I'm thinking about how that person made me really mad and I can't really process through my anger and this incident happened in specials first thing in the morning and it's 2:30 in the afternoon and I'm still not over it even though we've mediated and everything else. And I'm still not over it so I'm not ready to be in the position where I'm being friendly and respectful." And that's been very hard and we've had to really stress this year that we are the family and you don't have to like everyone but you do have to be respectful to everyone and you don't have the right to take away someone's learning opportunities just like you should not be sabotaging your own learning opportunities. And we've had a lot of talks, where like we talk about, "I can't fix what's going on at home but I can give you... I can empower you through education and that can give you a tool to move away from what's happening at home eventually." And so we're definitely better than we were used to be, especially science experiments. [The experiment] that we did, it would have been... there would have been fistfights. There would have been throwing of materials. The arguments that were happening during this time were very minimal compared to where we started from. So like even though it's annoying and irritating, like, "Just try this. Not everything has to be an argument not everything has to be a battle." We've come so far to whereas, it's not... nobody threw materials at anybody. Nobody stomped out of the room and screamed and that's what we are dealing with a lot at the beginning of the year. (Holly, Interview 2). Susan who works at the same school as Holly, states that the issues taking her time are the encouragement for teachers to use technology and the fact that the district has taken away textbooks for teachers and students. With these concerns along with a comment by Stephanie 
that there are fewer paraprofessionals to help in the classroom makes it evident that district finances are directly impacting the quality of education that the students are receiving.

Underused resources. Few teachers report relying upon traditional resources. Those with the most years of teaching experience report turning to these traditional resources less frequently. The youngest teacher in the group gives the most credence to the value of traditional resources whereas the older ones look inward. This means that the degree to which teachers use researchbased strategies depend upon their experience.

Environmental resources, such as the district ESL teacher and the building principal are rarely used. Several teachers reported using the district Parent Coordinator who is a former ESL teacher and who is fluent in French and Spanish. However, while all teachers mention that they would turn to the principal if they needed help, they provided no examples. Linda mentioned she would have support from her principal, but recognizes how busy she is and looks to solve her classroom concerns on her own.

\section{Strategies}

As discussed in chapter two, teaching strategies found to be effective with emergent bilingual students can be divided into the categories of Just Good Teaching (JGT) strategies and Linguistically Responsive Teaching (LRT) strategies. A pattern did emerge in the manner by which teachers spoke of the effective strategies they used with their emergent bilingual students. As teachers discussed the strategies they used with their emergent bilingual students, they would often quickly follow that up with a statement that this strategy is something they do with all their students.

Observations and interviews were coded according to research-based strategies that were considered JGT for all diverse learners and LRT, which are those specifically for emergent 
bilingual students. Teachers primarily use JGT. LRT strategies are only used to a limited degree. The strategies that they do use in LRT could be developed to a greater extent. While all teachers report or demonstrate using extra-linguistic supports, such as videos or graphic organizers, there were many examples when this could be done to a greater degree and more frequently and with more variety throughout the lesson to push the support from JGT to LRT.

Also, teachers rarely provided specific language learning strategies for students who are working at grade level or demonstrate what the teacher considers to be appropriate oral language. These teachers included Holly, Stephanie, Jennifer, and Melody. For example, Stephanie states that she does not provide specific strategies for her emergent bilingual student at a higher proficiency level because she scored in the top two scores in a beginning of the year Scantron benchmarking test. This test, however, does not provide an assessment for writing or speaking, two areas that emergent bilingual students need to develop in order to become fully proficient in English.

The data were analyzed according to whether or not strategies were useful for all students or emergent bilingual students in particular. It was found that teachers primarily used strategies useful for all students. A complete chart on the JGT and LRT strategies reported or observed by each teacher can be found in Appendix G.

\section{Combination Resources and Strategies}

Some resources and strategies overlapped. That is, what teachers may use as a strategy, later becomes a resource in her classroom. The opposite is also true. Such resource-strategies include knowledge of the student and empathy.

Knowledge of the students. Many teachers state that getting to know their students is a strategy that they use. Teachers use this knowledge of the students help them make decisions 
such as seating arrangements (Susan, Melody, and Linda). They use their knowledge of their students' home support system to determine what level of expectations they can hold for the effort their student put in outside of school (Linda). Teachers may also use what they perceive the child's proficiency level in English is in order to determine if they believe the child needs differentiated instruction (Holly, Stephanie).

Despite the knowledge of students being a key strategy that half the teacher participants report using (Susan, Melody, and Linda), several teachers described "knowledge" of the students that was inaccurate. At times the teachers seemed unaware of important information about their emergent bilingual students. Some examples of this important information that was lacking by the teachers is students' English proficiency levels, what their current levels are and what they need to know and do in order to pass the Assessing Comprehension and Communication in English State-to-State (ACCESS) test that demonstrates English proficiency.

In another example, some teachers were unsure which students qualify for services. Jennifer was unsure of the current standing in the program for one of her students who she reported speaks French and Arabic in the home. Melody, who is in the same school as Jennifer, believed that one child in her classroom had tested out of services and was pulled out for ESL services to help the other students.

All teachers report making efforts to include their students in the content from the beginning. They don't want them to feel different. Melody, for instance talked about how she hated to be singled out as a child and does her best not to single out any student within her classroom. Teachers who worked with newcomers described the challenge in keeping them involved in the curriculum despite the language difference. Stephanie said: 
I've recommended this to other teachers who get that look on that face when they have that first one that's extremely low, um English-wise, is just involve them from the get go... I've seen, I've seen ELL kids put in the hallway to work on something and they need to be in in everything. They need to be there. You just need to include them.

Holly said she didn't want them on the computer doing something different, but explained she didn't always know what to do.

Linda reported that she would, however, frequently put her newcomer in the hallway during math. She would say “He can’t do Science. He can’t do Social Studies” (Linda, Interview 1). She said that these activities would frustrate him, but made no mention of attempts to modify her lessons to reach him at his level nor were these attempts observed. She reported that during math class he was typically pulled into the hallway to complete basic math facts.

Linda also used her newcomer student as a way to reach a child with poor behavior. She would ask that child to be responsible for his learning.

Many teachers described high expectations for their students. Linda, however, stated that "I said he is at a 1, he's gonna be at a 1 pretty much the whole entire year and [the principal] was okay with that" (Linda, Interview 1). The 1 is in relation to the standards-based grading that the school district uses. This indicates "far below grade level." However, she also describes the importance of seeing where he is at and striving to make progress. She states:

I can judge [the newcomers and their families], I can get mad at them, or I can turn it around and see what I can do in the classroom to see progress. And I think um you know, I see it as growth as where are where did you start, you know, your baseline and then where do you grow from there. And he has grown (Linda, Interview 1). 
Linda sees the altered expectations for her newcomer student as a way of showing empathy to the child and his family.

Empathy. Like knowledge of students, empathy is both a resource and a strategy. Teachers use it to help determine how they should treat their students, but also try to develop empathy as a resource for their native English-speaking students. Linda talks about sharing with her students the fact that she speaks six languages so that they do not judge the newcomer immigrant students in a negative way for not speaking English proficiently.

Linda discusses the importance of building empathy with her American students for the newcomers and other immigrants in her class. She states,

It also helps to let the other kids know that you're trying to help that kid so they as a class they can see it so they wouldn't be picking on that kid, as 'You need to learn English because in this country...' I think that's really helpful to know that in this country it's okay to have more than one language" (Linda, Interview 2).

Her newcomer students help her to teach this value to her monolingual and American students.

Stephanie also works to share her empathy with her American students. She works with her newcomer students and uses her basic knowledge of French to teach the class how to count to ten in French. She states, "I think that's why [my American students] become so helpful because maybe some of them get that light bulb, like, 'I can't imagine learning everything I already know again in a different language"' (Stephanie, Interview 1). Stephanie's empathy is a resource. She fosters empathy in her students so that their desire to help her newcomer becomes a classroom strategy. 
Holly seems to have stumbled across the benefits of developing empathy with her students by accident as she read Esperanza Rising in a class with a student who spoke Spanish at home. She said:

[When] I chose it I didn't realize it had so much Spanish in it integrated throughout the book. And then, like 3 pages in, I realized that very fast. And so I tried my best with pronouncing the words and Miguel as so happy he was so excited he almost felt like we were kind of like it was opening up the whole class to Spanish and he would get excited because if I would say of word wrong he would jump into pronounce it right. But the kids, like, looked up to him like, this God. Like, "Miguel, knows this. Miguel knows all of this, like, everything that she's saying." (Holly, Interview 1).

She finishes this discussion by stating that this student emailed her this year, in $6^{\text {th }}$ grade, to say how much he had enjoyed reading that book with her when he was in $4^{\text {th }}$ grade.

\section{Quantitative Results for Research Question Two: What Resources and Strategies Do Teachers Draw Upon in Working with their Emergent Bilingual Students?}

To answer this question, I used descriptive statistics to demonstrate the resources and strategies that teachers use most frequently. Each of the different types of resources and strategies were assigned a number based on the frequency that the teachers report that they use that resource or strategy. I will first present a table demonstrating the mean use of resources that teachers call upon divided into groups based on teacher characteristics followed by a brief discussion of the conclusions. I will then follow this with a table and discussion of teacher strategy use, broken down by the teacher characteristics. 
Table 9

Teacher Resource Use

\begin{tabular}{lcccc} 
Resource Type & Mean & Standard & Range & Minimum/ \\
& & Deviation & & Maximum \\
\hline Personal & 11.74 & 8.07 & 49 & $5-54$ \\
Environmental & 8.86 & 3.13 & 16 & $0-16$ \\
Conventional & 6.67 & 2.71 & 17 & $0-17$ \\
\hline
\end{tabular}

The data presented above indicate that personal resources were the most often called upon resource by teachers with conventional resources being the least often used resource.

The following tables demonstrate the resources that teachers call upon. I divided the resources into the categories of personal, environmental, and traditional. The survey did not ask teachers who report they have never worked with ESL students to complete these questions on their survey.

Table 10

Mean Resource Use and Teacher Characteristics

\begin{tabular}{lccc}
\hline & Personal & Environmental & Conventional \\
\cline { 1 - 3 } Teaching Position & & & \\
Classroom teacher & 11.2 & 9.0 & 6.2 \\
Specials teacher & 10.7 & 8.7 & 6.7 \\
Other & 13.0 & 8.6 & 7.6 \\
Teacher Travel & & & \\
Never & 10.2 & 9.1 & 6.2 \\
Less than 1 month & 10.2 & 8.6 & 6.3 \\
2-5 months & 11.1 & 8.8 & 6.4 \\
6-12 months & 12.3 & 11.7 & 9.5 \\
More than 12 months & 33.5 & 11.0 & 7.5
\end{tabular}




\begin{tabular}{|c|c|c|c|}
\hline & Personal & Environmental & Conventional \\
\hline \multicolumn{4}{|l|}{ Bilingual Status } \\
\hline No & 10.5 & 8.2 & 6.1 \\
\hline Somewhat & 11.4 & 10.6 & 7.7 \\
\hline Fluently & 23.8 & 9.3 & 3.4 \\
\hline \multicolumn{4}{|l|}{ Teaching Experience } \\
\hline $1-5$ years & 11.2 & 9.1 & 6.9 \\
\hline $6-10$ years & 11.4 & 8.8 & 6.4 \\
\hline 11-15 years & 11.1 & 8.6 & 8.0 \\
\hline $16-20$ years & 8.8 & 8.8 & 5.8 \\
\hline More than 20 years & 11.0 & 16.0 & 7.0 \\
\hline \multicolumn{4}{|l|}{ Teaching in District } \\
\hline $1-5$ years & 12.8 & 9.0 & 6.7 \\
\hline $6-10$ years & 10.9 & 8.3 & 6.1 \\
\hline 11-15 years & 9.8 & 8.5 & 8.3 \\
\hline $16-20$ years & 9.7 & 8.0 & 6.4 \\
\hline More than 20 years & 14.3 & 9.8 & 5.9 \\
\hline \multicolumn{4}{|l|}{ Grade Level } \\
\hline Pre-K to $2^{\text {nd }}$ & 10.3 & 9.4 & 6.1 \\
\hline $3^{\text {rd }}$ to $5^{\text {th }}$ & 13.7 & 9.4 & 6.3 \\
\hline $6^{\text {th }}$ to $8^{\text {th }}$ & 9.5 & 7.8 & 7.0 \\
\hline $9^{\text {th }}$ to $12^{\text {th }}$ & 10.4 & 8.1 & 6.8 \\
\hline \multicolumn{4}{|l|}{ Experience with E.B. } \\
\hline 5 or fewer & 14.7 & 6.5 & 5.5 \\
\hline $6-10$ & 10.2 & 8.7 & 5.8 \\
\hline $11-15$ & 9.4 & 10.6 & 6.3 \\
\hline $16-20$ & 10.2 & 9.4 & 6.2 \\
\hline 20 or more & 11.9 & 10.2 & 9.0 \\
\hline \multicolumn{4}{|l|}{ Training with E.B. } \\
\hline None & 11.2 & 9.1 & 6.9 \\
\hline In-Service & 11.4 & 8.8 & 6.4 \\
\hline College/graduate school & 11.1 & 8.6 & 8.0 \\
\hline Conference/workshop & 8.8 & 8.8 & 5.8 \\
\hline Other & 11.0 & 16.0 & 7.0 \\
\hline
\end{tabular}


According to the data presented above, personal resources are the most commonly used resources by teachers in almost all teacher categories. It is highest in teachers who have lived out of the country for over one year, who have over 20 years of teaching experience, and who have worked with five or fewer emergent bilingual students. Meanwhile, teachers who have worked with 20 or more emergent bilingual students report the greatest use of conventional resources of all the teacher groups apart from one. That group are the teachers who report having spent 6-12 months living in a country that speaks a language other than English.

The least frequently used resources are conventional resources. The groups reporting the lowest use of conventional resources are teachers who have worked with ten or fewer emergent bilingual students and teachers with over 20 years of experience.

Table 11

Teacher Strategy Use

\begin{tabular}{lcccc}
\hline Resource & Mean & Standard & Range & Minimum/ \\
& & Deviation & & Maximum \\
\hline JGT & 40.83 & 13.69 & 50 & $0-50$ \\
LRT & 34.07 & 12.09 & 50 & $0-50$ \\
\hline
\end{tabular}

It is evident from the table above that JGT strategies are used to a much greater degree than the LRT strategies. However, the table below further break down the data to how different groups of teachers may use JGT and LRT strategies. 
Table 12

Mean Strategy Use and Teacher Characteristics

\begin{tabular}{|c|c|c|}
\hline & JGT & LRT \\
\hline \multicolumn{3}{|l|}{ Teaching Position } \\
\hline Classroom teacher & 43.3 & 34.8 \\
\hline Specials teacher & 40.0 & 34.7 \\
\hline Other & 32.8 & 36.7 \\
\hline \multicolumn{3}{|l|}{ Teacher Travel } \\
\hline Never & 43.7 & 34.9 \\
\hline Less than 1 month & 37.5 & 31.2 \\
\hline 2-5 months & 46.0 & 42.0 \\
\hline 6-12 months & 48.0 & 44.5 \\
\hline More than 12 months & 45.0 & 50.0 \\
\hline \multicolumn{3}{|l|}{ Bilingual Status } \\
\hline No & 40.3 & 35.5 \\
\hline Somewhat & 40.7 & 35.4 \\
\hline Fluently & 45.8 & 41.3 \\
\hline \multicolumn{3}{|l|}{ Teaching Experience } \\
\hline $1-5$ years & 42.5 & 35.0 \\
\hline $6-10$ years & 35.8 & 31.6 \\
\hline $11-15$ years & 41.0 & 33.6 \\
\hline $16-20$ years & 40.0 & 33.3 \\
\hline More than 20 years & 43.5 & 35.9 \\
\hline \multicolumn{3}{|l|}{ Teaching in District } \\
\hline $1-5$ years & 39.2 & 33.7 \\
\hline $6-10$ years & 38.1 & 31.9 \\
\hline $11-15$ years & 45.4 & 36.3 \\
\hline $16-20$ years & 41.0 & 33.9 \\
\hline More than 20 years & 42.5 & 34.8 \\
\hline \multicolumn{3}{|l|}{ Grade Level } \\
\hline Pre-K to $2^{\text {nd }}$ & 41.8 & 33.4 \\
\hline $3^{\text {rd }}$ to $5^{\text {th }}$ & 42.8 & 34.65 \\
\hline $6^{\text {th }}$ to $8^{\text {th }}$ & 36.0 & 32.5 \\
\hline $9^{\text {th }}$ to $12^{\text {th }}$ & 38.8 & 33.1 \\
\hline
\end{tabular}

Table Continues 


\begin{tabular}{lcc}
\hline & JGT & LRT \\
\cline { 1 - 2 } Experience with E.B. & & \\
5 or fewer & 31.4 & 26.1 \\
6-10 & 39.7 & 31.7 \\
$11-15$ & 48.1 & 37.4 \\
$16-20$ & 45.8 & 38.2 \\
20 or more & 41.3 & 45.9 \\
Training with E.B. & & \\
None & 42.5 & 35.3 \\
In-Service & 41.4 & 33.9 \\
College/graduate school & 41.6 & 38.1 \\
Conference/workshop & 46.3 & 35.8 \\
Other & 46.0 & 42.0 \\
\hline
\end{tabular}

From the data above, it is apparent that the JGT strategies are overwhelmingly the preferred strategies used by the majority of teaching groups. Only three categories of teachers report a higher use of LRT strategies. These are those who have spent more than 12 months outside of the United States, teachers who have experience working with over 20 emergent bilingual students and teachers who fall under the "other" category for teaching position, meaning that they are not a classroom teacher or specials teacher. These last two categories can also include the district ESL teachers who would likely have more knowledge of effective teaching strategies for emergent bilingual students.

Teachers with the most amount of experience with emergent bilingual students are those who both more frequently use LRT strategies as well as the greatest use of conventional resource. Teachers who have spent over 12 months in a country that speaks a language other than English are those who use the greatest amount of personal resources with a mean score of 33.5 and also those who report the most frequent use of LRT strategies with a mean score of 50.0. Furthermore, the data from the travel group indicates that that, apart from teachers who report 
they have never been to a country that speaks a language other than English, the use of LRT strategies continued to increase based on the amount of time that a teacher spent in a country that speaks a language other than English

\section{Qualitative Findings for Research Question Three: How Does a Teacher's Disposition Inform the Resources and Strategies They Draw Upon in Working with Emergent}

\section{Bilingual Students?}

The answer to this question is a bit more complicated than the question implies. Dispositions are complicated. There have been few studies, if any, that consider teacher dispositions toward the emergent bilingual students as a complicated and multifaceted construct. Rather, the literature describes dispositions as either positive or negative. That's too simplistic. People are complicated as are their opinions, attitudes, tastes, preferences, and, yes, dispositions. Therefore, a teacher may look positively on what an emergent bilingual student can offer their classmates, in learning about their community in a global context. However, that same teacher may look negatively upon the extra work of differentiation that the emergent bilingual student places on the shoulders of the teacher. Even though I was able to categorize most participants as positive, neutral/positive, or neutral/negative, there were times when Jennifer, with a neutral/positive disposition, made statements that could be perceived as negative (such as when talking about a former student who "couldn't read"). Similarly, Holly who had very positive dispositions towards emergent bilingual students had expressed that perhaps she would not be a good candidate for the research because her emergent bilingual student reflected a higher English proficiency indicating that she still was unsure what constituted an emergent bilingual student.

This research question implies that the teacher's dispositions may play a role in determining the resources and strategies that teachers use. This does seem to be the case to some 
extent. However, it also appears to be true that the resources and strategies that teachers use may impact their dispositions.

\section{Dispositions Affect Use of Resources and Strategies}

Holly was identified as one teacher who has an explicitly positive disposition toward emergent bilingual students. As mentioned, Holly states that her experiences traveling, hosting a foreign exchange student, and marrying a Mexican immigrant have created a great enthusiasm for learning about different cultures. She has a desire to reach her students and communicate to them how important they are as individuals to her and how invested she also is in their home cultures. This positive disposition, leads her to seek out how to best serve her emergent bilingual students. She uses the most traditional resources as well as uses her bilingual educator husband as an informal mentor.

Both Holly and Stephanie, with the most positive dispositions toward emergent bilingual students. They both also were the only ones to seek out help from the district ESL teachers or, in Stephanie's case, a volunteer with a background in ESL and foreign language education as a former high school German teacher. In this way, their choice of resources demonstrates a recognition that emergent bilingual students present a need for specific language-related knowledge and strategy use by teachers.

Susan and Jennifer both have positive dispositions toward all students. They primarily work to serve the needs of their emergent bilingual students through JGT strategies as they would with others students with diverse learning needs in their classrooms. They use small groups to focus on individual learning needs, model and practice comprehension strategies, highlight important information, and other JGT strategies. 
Linda's disposition was described as complex because she simultaneously empathized with her student and made it a point to introduce new languages and cultures to her native English-speaking students (which is a form of demonstrating respect). However, she also mentioned several times all the things she believes that Stephan "can't" do. This disposition led her to remove him from the classroom and have others work with him until he could do the same work as his peers. She gives this student one-on-one attention, but mostly delegates that task to others. However, she does not modify teaching to reach him at his level. Despite the complexity of her disposition, in the whole group setting, she used many strategies that would be useful specifically for emergent bilingual students. She checked in often, used one of the home languages with him, and provided some one-on-one instruction. While her disposition reflects a complex mix of deficit and non-deficit ideology, her teaching also reflects a mix of pushing him out of her room while also demonstrating respect for his culture and language.

\section{Resources and Strategies Affect Dispositions}

It also appears that selected resources or strategies may affect a teacher's disposition. For example, Stephanie selected a resource person as someone who was knowledgeable in second language acquisition. She then also demonstrated an understanding of what her emergent bilingual students may be going through. While she acknowledged feeling trepidation knowing that she would be assigned an emergent bilingual student, she now expresses confidence and joy in working with this population of learners.

As previously mentioned in the discussion of the first research question, every one of the teachers responded that they are pleased to have emergent bilingual students in their classes. Yet, teachers have also expressed apprehension in their first year of teaching emergent bilingual students regarding their ability to teach them, give them challenging work, meet them where they 
are, and move them forward. If teachers adopt differentiation strategies in their classrooms, a newcomer emergent bilingual student presents an additional work load and may therefore bring a more negative attitude if the teacher feels that he or she cannot address the child's needs. However, when the needs of newcomer students are not addressed in a differentiated manner (as with Melody who believes in treating all students the same) or are addressed primarily through the use of outside volunteers (as is the case with Linda) teachers may then maintain a more positive disposition than they may otherwise present if they felt they were responsible for providing that instruction because their choice to not differentiate or to delegate the work of teaching to others is minimized.

\section{What May be More Influential on Resource and Strategy use than Teacher}

\section{Disposition?}

What appears to be more influential on resource and strategy use than teacher dispositions are their beliefs about how languages are learned, what assumptions teachers arrive at from the way they empathize with their students, what they believe they know about their students in general and their students' needs, the way they view their role as a classroom teacher, and the other challenges teachers face in their classrooms. I will speak about each of these briefly below.

Beliefs about how languages are learned. Teachers seem to hold different beliefs about how languages are learned. Stephanie suggested that she occasionally wants to give her student content information in the home language, but says, "We would try to find things on the Chrome books that were appropriate and I learned not to put them on French-speaking things because that's not what they need. They need that immersion in the English language and so books read to them and those kinds of things (Stephanie, Interview 1). This belief about how languages are 
learned, the accuracy of which will be discussed in chapter five, led to a strategy to provide English-only education even when support in the L1 is available. (Stephanie, Interview 1).

Similarly, Melody states that she does not differentiate her instruction. She believes that it is best for all students to just "go in full force" and states that "by the end of the year, you would never know they started the year [at a lower English proficiency level]." Again, this belief in the efficacy of an immersion experience relieves Melody of an obligation to differentiate instruction.

Linda, the only case study participant who is also a former ESL student, states that she learned English through an ESL program in junior high. As a newcomer herself, her $6^{\text {th }}$ grade teacher would send her out of the classroom to learn. While she is careful to make sure that her student feels a strong part of the classroom community, she has also adopted the practice of sending her newcomer student out of the classroom with volunteers, aides, and student teachers so as to provide the individualized instruction in basic math facts and reading that she believes he needs. Again, her belief about how languages are learned, based on her own experiences, leads her to provide teaching strategies that reflect these beliefs.

Teacher empathy. The way that teachers empathize with their emergent bilingual students and imagine how they feel, leads them to either differentiate to a greater or lesser extent. For example, Melody states that she never wanted to be singled out or seen as different when she was a student. Therefore, if she provides an accommodation for one student, she wants to do this for all students. When one student had a disability that required her to use the bathrooms at any time without asking, she made that accommodation for all of her students. Melody carries her fear of singling out students for their differences to her bilingual and emergent bilingual students as well. She states, "I think it's also nice that they get to keep [their home language] to 
themselves and use it when they need to and feel comfortable here as well” In this way she feels that she is helping her students by restricting their language use in the classroom as opposed to capitalizing on the funds of knowledge the students bring to the classroom.

Holly has also talked about her husband's experience as a newcomer when he was in the fourth grade. She reports that she frequently asks her husband to consider her practices in light of his experiences. She describes her search for his advice in this way:

How would you feel? How would you feel if this happened? Because I never want to put my kids in the position where they feel left out or different or anything like that and so I've gone to him quite frequently. "Hey, you know, in your experience how would this have made you feel? Or, how does this come off as would this be embarrassing or too hard? And he's not my [student] but he's obviously got some insights; insights that I trust. (Holly, Interview 1).

In this way, her husband's experience and her use of him as a resource influences the strategies she chooses to employ with her students. This is done despite the fact that his perspective represents only the story of one immigrant from one culture over 20 years ago.

Linda often talks about her experience as an emergent bilingual student when discussing her students. She is aware that it can feel uncomfortable to feel lonely and separated from the class. She describes when her $6^{\text {th }}$ grade teacher sent her to a second-grade classroom because "she didn't know what to do with the me" (Linda, Interview 1). Linda states:

Many of it has to do with the fact that I came from a background where I, you know my sixth-grade teacher left me alone in the corner. She actually sent me to a second-grade class thinking that maybe I was I would be suited in second grade you know so I was l'k'"5'7'" big girl going to second grade with little munchkins for a whole week and the 
kids at the beginning thought, "Oh my god we have an aide in here," you know. And when they realized I couldn't speak to them they started leaving, like, skedaddle, you know. Like literally scattered kids. And so my experience has helped me become a better teacher, to become a better strategist for [my ESL student], for all the students who come into my classroom. And have more empathy for them to see that they are struggling and to be able to see that look their parents have to work to make a living and the' don't have that support at home. (Linda, Interview 1).

Based on how she is empathizing with her student, she is cautious about making sure he feels comfortable as a part of the classroom. She takes pride in the way in which her American students take Stephan under their wing to help him. However, she allows him to feel a part of the classroom culture while also providing what she believes are appropriate strategies at his level that are different from what the rest of the class is doing.

Jennifer made no mention of a fear of singling out students. I also observed her using small group instruction to her emergent bilingual students. Susan also made no mention of singling out students. Similarly, she used small group instruction with her emergent bilingual students, reported placing these students in differentiated reading groups, and reports that she reads tests aloud to her emergent bilingual students and anyone who requests it. Again, Stephanie does not report a fear of singling students out and reports reading tests aloud and working in small groups with them. She also states that the biggest advice she would give to teachers is to always include the emergent bilingual students and not to send them to the hallway or have them doing different work. While these teachers did express compassion for their emergent bilingual students, the way they empathized with their students did not extend to feeling bad for students for being different from American peers or giving them lower-level work. Rather, these teachers 
chose to showcase their unique learning strengths and providing the instruction students needed while giving access to the general curriculum.

Beliefs about students in general. Susan, Linda, and Jennifer report similarities between their emergent bilingual students and their native English students in that all the learners in their classroom are unique and have their own specific learning styles and needs. These teachers incorporate their emergent bilingual students into some of the same differentiation strategies they use for their American students. They provide a check-ins with students and one-on-one time. In this sense, this belief that all students learn differently leads them to try teaching strategies that meet these different needs.

Role of the classroom teacher. Teachers appear to have different ideas of what their role as the teacher actually is. Some teachers seem to believe that the teacher's role is to teach the assigned grade level curriculum. Those who need additional help will receive it through interventions, the ESL teacher, or other means. Linda is one example of this latter belief. She stresses the importance of community and empathy in her teaching, but holds deficit beliefs of what her newcomer student can and cannot do as a result of his language or prior academic experiences. She states that she does her best to meet him where he is at and move him forward. However, in order to accomplish the task of meeting his individual needs, she has volunteers, aides, and a student teacher pull him out during instruction time which results in an unequal exposure to the mandated curriculum than what the other students receive. While Linda has talked about working with Stephan individually, she has also said that she leaves the reading instruction to her volunteer. Therefore, because she seems to delegate specific teaching tasks to others, it appears that this delegation influences the teaching strategies and resources that she uses. 
Jennifer and Susan on the other hand have each stated that they recognize that all students have specific educational needs. They approach teaching by spending time working with individual students and working in small groups with emergent bilingual students. Because of this approach to teaching, they appear willing to work with emergent bilingual students individually, check in often, encourage (or at least not restrict) the use of their L1 in the learning process, use guided reading groups, small group instruction, and reading tests or directions out loud. These activities are done while the students are expected to participate to the highest degree that they are able.

Beliefs about one's own nation in relation to other nations. While teachers may state that they are happy to work with emergent bilingual students, an Amer-centric ideology may result in teaching strategies that quietly reject the child's home culture and language. For example, Melody stated that students should keep their home language to themselves. She also expressed a mocking attitude toward her visit to a foreign country. Melody believed that teachers should "go in full force" when teaching emergent bilingual students, which was her way of describing an immersion system for learning a language or learning content through English. These beliefs meant that she does not modify assignments, texts, lessons, or assessments to aide her emergent bilingual students. She gives preference to strategies that work for all of her students. Melody makes the assumption that students do not want to stand out because their language or culture may be different from the language and culture of other students in the class. However, students may be proud of their home language or heritage. Silencing this side of the students may subtly communicate to the child that only English and the American culture is valued in the school. 
Fear of being different. Melody also states that she did not want any of her students to stand out. Therefore, she would rarely pull them into groups unless she was going to work with all her students in a group because she doesn't want them to feel different. She states:

I want them to feel as part of the classroom as much as anybody else. And I make the modifications for all my students not just, you know, ESL students... If I see where [my ESL students] were falling behind then of course 'here's going to be more one-on-o'e. I'm not going to make that obvious. I probably wouldn't pull them just by themselves that day. It might be, you know, Minh at 10:30 and at 10:40 I might have my regular education students in here that are completely proficient and go from there. But ' don't ' don't like the singling out portion of that, I guess, is what I'm trying to say. (Melody, Interview 2).

She also states:

I don't really see [emergent bilingual students] any differently. Obviously, I see a form that comes through that they get services. And they may not know every part of the language and it just seems to work. And by the end of the year you would never know that that student had started the class that way. (Interview 2).

While Melody may believe that she is doing her students a favor by not seeing their differences in cultures and languages, the truth is that culture and language are an integral part of the students' identities and must be acknowledged and honored in the classroom.

Melody's stance on what she calls singling children out extend to non-emergent bilingual students as well. Her rules for one child are the rules for all. Melody went on to tell a story about a non-emergent bilingual child who had medical issues in her classroom. Due to the medical 
issues the child should be allowed to go to the bathroom without asking. However, because of the needs of this child, she changed the rules for her entire class. She says:

Because I had some students with medical issues, [students] can have [bathroom] passes on their desk and then go. But I keep telling them, so if I have the same students going like ten times, I know that's not an issue. But I do it more for my student that may have a medical issue. Or if you're sick, you don't ask at all you just get up and go and try to get to the bathroom to not expose everyone. (Melody, Interview 1).

Regardless of the reason for this particular rule, according to Melody, any rule in M'lody's class applies to all children

This strategy of Melody's may be a result of a deficit ideology that suggests that children will perform in school up to their abilities. Success is a result of student abilities and the effort they apply. Those who are able succeed, while those who are unable, even if it is a result of linguistic differences, will not succeed. Rather than give children with medical needs and linguistic needs the individualized help they require, Melody makes an effort to keep her rules and her instruction the same for everyone so that no one feels different from the group.

On the other hand, Melody's practice of keeping instruction the same for all children may not be a result of deficit ideology. Rather, she claims that it is because she herself did not like to be called upon to read in class. Like Linda and Holly, she compares her school experience to that of her students which led her to the conclusion that standing out is wrong. She says:

I shared that my personal experiences when I was in school in this classroom. I was called out to read and I didn't like to read when I was in third grade. I get very shy and nervous and stumble across my words and then I'd get up and I was done for the day because if I get nervous I shut down. (Melody, Interview 2). 
Perhaps her strategy is more of a result of the way she empathizes with her students rather than the deficit ideology outlined above.

\section{Quantitative Results for Research Question Three: How Does a Teacher's Disposition Inform the Resources and Strategies They Draw Upon in Working with Emergent Bilingual Students?}

As in the questions above, we will start by looking at teacher resource use. A linear regression was conducted to evaluate the null hypothesis that a teacher's disposition toward his or her emergent bilingual students has no impact on the resources teachers use in teaching. The independent variable in this question was the LATS score while the resources that a teacher uses is the dependent variable. A linear regression was run for all three types of resources. That is, the

uses of personal, environmental, and conventional resources were each examined individually. A significant regression equation was not found for the use of personal resources.

However, a significant relationship was found for the use of environmental resources and the LATS score. In order for a relationship to be significant, the $p$-value must be lower than .05 . The $p$-value for these two variables is .000 , which is lower than .05. A linear regression analysis was conducted to evaluate the effect of the LATS score on the teacher's reported use of environmental resources (the more negative disposition as indicated by a higher LATS score, the fewer environmental resources the teacher called upon). A significant regression equation was found $(\mathrm{F}(1,55)=25.165, \mathrm{p}=.000)$, with an $\mathrm{R}^{2}$ of .31. Participants' use of environmental resources is equal to $15.69+(-.25)$ of the LATS score. Participants' average number of environmental resource use decreased .25 for every increase in the teacher's negative disposition as measured by the LATS scale. 
A significant relationship was also found for the use of conventional resources and the LATS score. In order for a relationship to be significant, the p-value must be lower than .05 . The $p$-value for these two variables is .000 , which is lower than .05. A linear regression analysis was conducted to evaluate the effect of the LATS score on the teacher's reported use of environmental resources (the more negative disposition as indicated by a higher LATS score, the fewer conventional resources the teacher called upon). A significant regression equation was found $(F(1,55)=24.878, p=.00)$, with an $R^{2}$ of .31. Participants' use of conventional resources is equal to $12.57+(-.22)$ of the LATS score. Participants' average number of conventional resource use decreased 0.2 for every increase in the teacher's negative disposition as measured by the LATS scale.

A linear regression was conducted to evaluate the null hypothesis that a teacher's disposition toward his or her emergent bilingual students has no impact on the strategies teachers use in teaching. The independent variable in this question was the LATS score while the teacher's use of LRT or JGT strategies that a teacher uses are the dependent variables.

A significant relationship was found for the use of JGT strategies and the LATS score. In order for a relationship to be significant, the $p$-value must be lower than 0.05 . The $p$-value for these two variables is .000 , which is lower than .05 . A linear regression analysis was conducted to evaluate the effect of the LATS score on the teacher's reported use of just good teaching strategies (the more negative disposition as indicated by a higher LATS score, the fewer just good teaching strategies the teacher called upon). A significant regression equation was found $(\mathrm{F}(1,55)=15.96, \mathrm{p}=.000)$, with an $\mathrm{R}^{2}$ of .22. Participants' use of just good teaching strategies is equal to $66.20+(-.92)$ of the LATS score. Participants' average number of just good teaching 
strategies decreased .92 for every increase in the teacher's negative disposition as measured by the LATS scale.

A significant relationship was also found for the use of LRT strategies and the LATS score. In order for a relationship to be significant, the $p$-value must be lower than .05. The $p$ value for these two variables is 0.00 , which is lower than .05 . A linear regression analysis was conducted to evaluate the effect of the LATS score on the teacher's reported use of linguistically responsive teaching strategies (the more negative disposition as indicated by a higher LATS score, the fewer linguistically responsive teaching strategies the teacher called upon). A significant regression equation was found $(\mathrm{F}(1,56)=25.97, \mathrm{p}=.000)$, with an $\mathrm{R}^{2}$ of .32 . Participants' use of linguistically responsive teaching strategies is equal to $60.832+(-.973)$ of the LATS score. Participants' average number of just good teaching strategies decreased .973 for every increase in the teacher's negative disposition as measured by the LATS scale.

\section{Qualitative Findings for Research Question Four: What is the Interplay Between the Teaching Strategies and Resources that Teachers Use in Working with Emergent Bilingual Students?}

In taking a closer look at the strategies and resources teachers use, three things became evident. First of all, some personal resources that teachers carry with them into the classroom lead to a more frequently reported or observed use of LRT strategies. Secondly, the line between what constitutes a resource or a strategy can be blurred. Teachers may use a strategy that later becomes a resource, or may have a resource that can be used as a strategy. However, an inaccurate resource, such as faulty knowledge of the student, may lead to ineffective strategies. Finally, a lack of district financial resources may impact classroom resources and therefore 
teacher strategies in unequal ways, with those schools with the lower SES population most affected.

\section{JGT and LRT Strategies and their Connection to Resources}

There are more JGT strategies than LRT strategies that can be observed or reported. Therefore, logic dictates that more JGT strategies would be present in the classrooms. This was the case for our participating teachers. Therefore, I looked specifically at the use of LRT strategies to determine if there was a pattern or a connection between resource and LRT strategies.

Of the LRT strategies, all qualitative participants report or were observed using extralinguistic supports and all but Susan report or were observed using physical gestures to communicate ideas. However, only teachers who expressed a strong interest in other cultures (Holly and Linda) and had the personal resource of direct connections with bilingual individuals used the LRT strategy of specifically acknowledging and honoring the emergent bilingual child's culture. For example, only Holly and Linda discussed or were observed using strategies that allow students to compare the home culture to American culture. It was only these two teachers, again Holly and Linda, who had the personal resource of learning a second language to a level of comfort that used this resource to explicitly teach cognates. The only teacher to have the personal resource of learning English through an ESL program, Linda, was also the only teacher who reported explicitly teaching American sentence structure. Only teachers who expressed the personal resource of an interest in cultures, not just travel, (Stephanie, Holly, and Linda) reported or demonstrated that they took time outside of school hours to learn about their emergent bilingual student's culture and language. These three were also the only teachers who reported that they allow their students to teach the other children about their culture either through sharing 
a presentation about their home country or teaching American students words in the child's home language.

\section{Resources as Strategies/Strategies as Resources}

There was a fluid movement between some resources and strategies that teachers use. Mentors and teacher empathy are two resources that some teachers reported calling upon. These resources were then turned into a strategy in the classroom. Some teachers also report taking time to get to know their students as a strategy that they use. This knowledge then becomes a resource for the teachers in helping them determine what they consider the best way to instruct that child. This also means that the people who teachers turn to as a mentor and the way they use the mentor impacts the strategies teachers use. Likewise, the way teachers empathize with their students affects the strategies they employ. Similarly, the strategy of getting to know students, regardless of whether or not the "knowledge" is accurate becomes a resource for making curricular decisions.

Mentors as resource turned strategy. Mentors who are knowledgeable of the way languages are learned lead to teachers who are more informed on the individual needs of emergent bilingual students. Mentors who have specific knowledge of reading strategies can also share this knowledge with teachers. This shared knowledge can only take place if the teacher allows this to happen.

Stephanie and Linda are good examples. Stephanie turned to a friend and former colleague as a mentor to seek advice on working with her emergent bilingual student. This mentor also taught German at the high school level and taught adult ESL. She shared resources and strategies that Stephanie then implemented in her own classroom. Step'anie's mentor also came to the classroom to read to and specifically work with Step'anie's first emergent bilingual 
student. While working with that student, the mentor, Christina, provided Stephanie with ideas of strategies to employ with that child. In this manner, Step'anie's choice of a mentor resulted in specific strategies that she adopted in her teaching.

Linda, on the other hand, selected a resource person who was a neighbor with a reading specialist endorsement. Linda approached Ms. Amy as a resource who could do the work of teaching reading to her emergent bilingual student rather than a resource for Linda to learn about effective teaching strategies. Therefore, Linda's approach to her mentor did not result in improvements in teaching strategies for Linda because she did not choose to use her in that manner.

Empathy as a resource turned strategy. While personal experience does seem to be associated with a more positive disposition toward emergent bilingual students, empathy can lead to teachers making assumptions on the needs of emergent bilingual students assuming that their experiences will be or should be similar. For example, Linda is the only teacher who shares an experience with her emergent bilingual students of coming to the United States as a child and having to learn the English language and content at the same time. She compared her experience to that of her newcomer student several times. At one point she comments that she learned differently from this child. Linda discusses spending a day each week in the summer to work with one of her newcomer students. She states:

I would help him a day over that summer, you know. But he wouldn't... But, I know... my learning experiences are very different from other people's learning experiences. My learning experience was like, I was driven. I had literally 3 [to] 4 hours of work on the floor. And I had to go through different dictionaries and trying to learn it. For him he didn't have that support. He didn't have the educational background that I had. He came. 
He didn't know how to read. He didn't know how to do math, you know. He was different. I was just... So, you know, I couldn't expect the same thing from him. Linda's focus here is on the differences between the way she approached learning English and how her student approaches learning English. She highlights what he cannot do to express empathy for how their academic resources differed. In this fashion, Linda demonstrates lower expectations for her student than she had for herself while learning English because of the difference in academic resources they had coming to this country.

Linda also empathizes with her students in how uncomfortable it can feel as a newcomer in the United States. She reports that she was determined to give her student a different experience of what she had when $\mathrm{sh}^{\mathrm{e}}$ arrived in 6th grade and was $\mathrm{p}^{\mathrm{ul}}$ led into a 2 nd grade classroom. As a result of this experience, she stresses community and inclusion amongst her students and teaches them that it is okay to know more than one language.

It appears that Linda's two ways of empathizing with her student that is presented here may conflict. These lower expectations are manifested in strategies that are directly against what she states she believes are best practices. That is, she pulls the child out of the class, out of where he would be exposed to the general curriculum. Instead, he is pulled out to work on basic recall and retell work, such as memorizing math facts. He also works on reading with the reading specialist resource person. In Linda's case, by assuming that Stephan would feel frustrated in science and social studies, she has relieved herself from her responsibility of teaching him the mandated content in a way he would understand.

Holly's personal experiences with travel and the stories she hears from her husband help her to empathize with her students. When unsure of how she should approach a learning task with a newcomer student, she goes to her husband. Holly acknowledges that her husband is not 
her students, but she does trust his insights. His culture, home language, and unique personal make-up make be differ from that of her students. If Holly recognizes this, she may be in a better position to analyze if his advice is suitable for her students. If not, she runs the risk of allowing her husband's experience to dictate decisions made for her students that may or may not be appropriate.

Although Melody does not have personal experiences as or connected to a bilingual student, she does use her empathy to make decisions on the way she approaches the cultural and linguistic differences in her classroom. As mentioned, Melody describes the way she did not like to be called out to read when she was a student. She expresses that she would not want to be in the position that her students are in by coming to a new country with a new culture and language. She believes that it is good for students to keep their home languages to themselves. With this style of empathy, she does not allow for any strategy that will build upon the child's existing linguistic resources. Due to the way that Melody empathizes with her students, she is making an assumption about their individual preferences. Perhaps Melody's students are proud of their linguistic and cultural heritage and would like an opportunity to be recognized for the funds of knowledge they bring into the classroom. Perhaps they would like an opportunity to share this with others in the way that Holly's student felt empowered when the class read Esperenza Rising.

Empathy as strategy turned resource. A second way that empathy becomes a strategy is the way four of the teachers strive to instill empathy in their general education students. Linda, Stephanie, Susan, and Holly all talk about the ways they encourage community within their classrooms. Their empathy for what the students experience leads to a desire to create an inclusive classroom environment. Linda describes with pride how her students take her 
newcomer student under their wings to help him and bring him into the classroom. She has taught all her students the alphabet in French, which is one of the languages spoken in her newcomer student's home country. Stephanie has also taught her entire class how to count in French as a way to help them identify the challenges her newcomer students are experiencing. She states that this experience is one reason why her students have become so helpful to her emergent bilingual students. In this way Linda and Stephanie have worked to share their empathy with their American students so that they can also be called upon to help teach the newcomer student.

Knowledge of students as a strategy turned resource. Resources and strategies have the ability to mix together. As mentioned earlier, knowledge of their students is a strategy that was named by three of the teachers. The knowledge they have of their students helps them make decisions on where they should sit, whom they should work with, and even what content their emergent bilingual students receive. In this sense, the strategy of getting to know students also becomes a resource to call upon to instruct those children. Similarly, the mentor that a teacher turns to in helping determine strategies for emergent bilingual students is a resource for learning languages that leads to the development of strategies.

However, it can be problematic if the knowledge is faulty or incomplete. While the district makes decisions on which teacher will be assigned to the emergent bilingual student, which ESL teacher will be assigned to the building, and the learning objectives that the general classroom teachers are to teach to, the state determines the amount of service time each emergent bilingual student should receive based on proficiency. That leaves the individual teachers to determine how they will have their students meet the learning objectives. They make curricular decisions regarding how the strategies they will use to meet the assigned learning objectives. 
With emergent bilingual students, teachers are also deciding what aspect of the curriculum their emergent bilingual students will be exposed to. These decisions are made based on the assessments that teachers make on their students and the information provided about each child. If this information is inaccurate or based on assumptions, they may impact the degree to which the emergent bilingual students are exposed to the general curriculum, the ability of the teacher to activate prior knowledge, and the methods the teachers use to meet their assigned learning objectives. Throughout this study, there were several misconceptions of teachers that were identified and several other potential misconceptions that were unable to be verified.

Teacher misconceptions and impact on teaching. Teachers did not always know 'heir students' country of origin or the languages that they primarily speak in the home. In discussing Linda's student, Edouard, who she used to have and his sister, her current student, Faith, she stated he was from the Cameroon and speaks either Lingala or French. While this may be possible, Lingala is a language that is primarily spoken in the Democratic Republic of the Congo, the home country to many of the students in this school. It is not a language that is commonly spoken in Cameroon.

Furthermore, it is important for teachers to know if the child's family prefers to speak French or if they prefer to speak Lingala in the home. With this knowledge, there is a better understanding of the interpersonal relationships between the other emergent bilingual students in the building. Linda reports that when Stephan was new to her classroom, she had him sit next to Faith who was also from Africa with the hopes that she could translate for him. However, if one child's family primarily speaks French and the other child's family primarily speaks Lingala, or if the family is from Cameroon, as she reports, but does not speak Lingala, she has unrealistic expectations for how these two children can interact. 
Misunderstanding in the development of language systems. The scenario described above with Linda's choice in partnering Stephan and Faith also demonstrates a misunderstanding of the development of language systems. While two children who live in the United States and are learning English and who also speak Lingala and French may seem to have similar language backgrounds. However, as children, they are still developing these three separate language systems. As mentioned in the previous paragraph, the development may depend upon the language primarily spoken in the home. It also depends upon the age of the child at the time of immigration. Linda mentioned that was three years old when she came to the United States and was place in an English-speaking preschool. Therefore, her knowledge of Lingala and French is likely not developed to the same level of Stephan who just immigrated to the United States this year. It is very likely not up to the task of translating fourth grade academic content. Therefore, the inaccurate knowledge of the students was a faulty resource that resulted in an ineffective strategy.

Misunderstanding of the procedures of the ESL program. Two teachers expressed a misunderstanding regarding the eligibility of their students for receiving ESL services. When asked how many emergent bilingual students Jennifer had in her class, she responded by stating she did not know if one of her students who speaks English, French, and Arabic "counted" because she does not receive services. After greater inquiry, Jennifer stated that while she does not receive services, she still has to take the state mandated test of English proficiency every year. If this is true, it would mean that she still qualifies for services, but her family has refused services or did not consent to services. If that were the case, that indicates that Jennifer is the only teacher who would have the opportunity to provide the child with English language instruction. This would be important information for the teacher to understand. 
Melody, who teaches at the same school as Jennifer, said that her student did not qualify for services, but was pulled out for ESL services with the ESL teacher because "she's come so far." She later said that the child did qualify for services. It sounds as if there is some confusion in this school over the students who qualify.

Misunderstanding on the role of the ESL teacher. Teachers also did not express a shared understanding of what the ESL teacher does. Because the ESL teacher is an environmental resource, a misunderstanding of what this teacher does means that the classroom teacher may not be using this resource correctly or unable to learn from the ESL teacher what strategies to adopt in the classroom. Linda is the only teacher with an accurate understanding of what the ESL teacher does. She understands that the ESL teacher is a teacher with specialized knowledge of second language acquisition. Stephanie suggested that the ESL teacher is a support for the classroom teacher. Therefore, she may not understand how the ESL teacher can be a support to her. Melody stated that the role of the ESL teacher is to build background knowledge for her emergent bilingual students and to find opportunities for the student to share her background with others. It is difficult to make the best use of the ESL teacher as a resource or strategy if the teachers do not know what they do or there is not a shared consensus of their role.

Misunderstanding of what constitutes an ESL student. Finally, teachers seemed to assume English proficiency based upon perceived speaking and listening fluency. For example, Stephanie and Holly indicated that their students do not need materials to be modified because they appear to have a strong language proficiency. For example, Stephani' said, “I don't give it really a second thought anymore as to what she's doing in the classroom because she's making it" (Stephanie, Interview 1). Holly also questioned if she should be a part of the study because, in her words, “[my emergent bilingual students] hardly [receive services] a' all so I don't I mean I 
do differentiate to some degree but not as much as necessarily...” (Holly, Interview 1). And yet, these students are still working on demonstrating proficiency at a level that would test them out of the ESL program. They still may need support in vocabulary, figurative language, and increased opportunities for reading and writing. Other teachers who did know their students' standing in the program still did not personally consider some students as ESL students if they demonstrated oral language proficiency and they appear to keep up with the class.

For example, at the beginning of her first interview, Holly expressed apprehension over her inclusion in the study because her students were hardly pulled out for services, indicating that their proficiency levels were high (Holly, Interview 1). Also, as mentioned, Jennifer did not understand whether or not Kadijah who speaks French, English, and Arabic would qualify as an emergent bilingual student. She stated that this particular student still was required to take the ACCESS test each year, which would mean that she is a student who refused ESL services. According to the Illinois State Board of Education, "ELs in Illinois need to receive an overall composite score of 4.8 on ACCESS to be English proficient" (Illinois State Board of Education, 2019). This means that while she is not taking ESL services from the ESL teacher, she is still learning the language and could benefit from specific teaching strategies to improve her English language development. Without this understanding, it may be safe to assume that the teacher is not providing instruction that uses those language learning-specific strategies (Jennifer, Interview 1).

\section{Resources That Allow for Strategies}

Some environmental resources can help teachers provide better strategies for their emergent bilingual students. These resources include paraprofessionals and textbooks. It also would include a classroom with students that have strong social and emotional understanding. 
However, with the lack of district financial resources, the teachers are impacted in different ways depending upon the socioeconomic population of the school.

Strong social emotional student level as a resource. Some teachers have an environmental resource of a greater percentage of students with a strong social and emotional development. Students with this resource allow teachers to provide learning opportunities that are more meaningful for emergent bilingual students. For example, when all students follow school directions fairly well, teachers are able to provide hands-on lessons and one-on-one support for emergent bilingual students. Jennifer, Melody, and Linda worked in schools with a higher SES student population. They did not report the same level of emotional needs of the schools of students with a lower SES population. These teachers were often seen to be working with students individually.

However, some teachers do not have this environmental resource. Stephanie described the emotional concerns of her students and how that takes away learning opportunities from the class as she uses time to provide comfort and security to these students. Holly described the work she puts into teaching her students that she is there for them and the classroom is a safe space for them. Susan, who teaches at the same school as Holly was observed to spend independent practice time not working in small groups, but rather giving hugs, comfort, and encouragement to students who had been acting out. These teachers are then limited in their ability to provide teaching strategies such as check-ins and one-on-one support.

Lack of district financial resources unevenly impacting students. Similarly, district financial resources determine whether or not teachers have aides in their classrooms which would help or hinder their ability to provide small group instruction. The missing district financial resources means that the district reduced the number of a paraprofessionals 
(environmental resource) available and stopped buying textbooks (conventional resource). These decisions therefore put more work on the teacher, depleting teachers of the resource of time (a personal resource). This leads to fewer opportunities to develop professionally in a teacher's own time.

\section{Conclusion}

This chapter presented the four research questions in through the lens of qualitative data. Additionally, during this phase of data collection, it was determined that while teachers consistently report JGT strategies, they may not use LRT strategies as frequently. Therefore, the survey distributed and discussed in chapter five included a portion on teaching strategies, some of which are JGT and some of which are LRT. These strategies were selected based upon what participants in the qualitative phase of data collection report using and are labeled as JGT or LRT based on the relevant literature, which can be found in chapter two. In the upcoming chapter, I will discuss the results of this survey and will provide data for answering the research questions based on quantitative research. 


\section{CHAPTER V: DISCUSSION}

The purpose of this study was to take a deeper look at the dispositions that teachers hold toward their emergent bilingual students. This work, builds upon past studies by examining teacher dispositions, both qualitatively and quantitatively, to determine the factors that contribute to these particular dispositions. The study goes further by looking at the resources that teachers call upon, as defined by Cohen, et al. (2003), and the teaching strategies that teachers employ when working with these students. Finally, the data provided insight into the interplay between the teachers' dispositions, resources and strategies. Specifically, the research questions ask:

1. What influences the dispositions that teachers hold toward their emergent bilingual students?

2. What resources and strategies do teachers draw upon in working with their emergent bilingual students?

3. How does a teacher's disposition inform the resources and strategies they draw upon in working with emergent bilingual students?

4. What is the interplay between the teaching strategies and resources that teachers use in working with emergent bilingual students?

In order to answer these questions, a sequential exploratory mixed methods approach was adopted. The study began with a phase of qualitative data collection involving six third and fourth grade teachers. The teachers were interviewed two times, observed teaching two times, and provided documents for analysis throughout the process. Data were analyzed and the findings of this stage contributed to the survey that was used for the second phase of data collection, which comprised of a survey distributed to 297 teachers and completed by 55, reflecting a response rate of $18.5 \%$. Findings for this study were discussed in chapter four. 
In the present chapter, I will elaborate on the findings in the order of the four research questions provided above. I will then provide conclusions that can be drawn from the study in the context of current research. Finally, I will offer implications of these conclusions, limitations of the research and recommendations for further study.

\section{Discussion for Research Question One: What Influences the Dispositions That Teachers Hold Toward Their Emergent Bilingual Students?}

Considering the qualitative and quantitative data together, there are a number of experiences that appear to positively impact a teacher's disposition toward working with their emergent bilingual students. The study findings suggest that the greater amount of exposure a teacher has to diverse cultures, the more positive that teacher's disposition is toward emergent bilingual students. This exposure can come in various forms, such as a personal relationship with someone who has experienced being an emergent bilingual student, increased training in working with emergent bilingual students, attempts at learning a foreign language, and experience teaching this population of learners.

The analyses of the qualitative data enabled me to pinpoint the individuals in two of the teachers' lives who influenced their perspective on the emergent bilingual students. In this study, the participants who felt this influence in their personal lives were Linda and Holly. Although Linda often left the teaching component of her emergent bilingual students to volunteers, the ESL teacher, and students in the classroom more than the other teachers chose to do so, she was adamant about the importance of making sure the emergent bilingual student felt that he or she was a part of the classroom culture based on her own personal experiences. For Holly, the relationship that was significant in influencing her perspective on working with emergent bilingual students was that of her husband who had been an emergent bilingual student herself. 
She mentioned that she often turns to him to ask how he might feel if a teacher had used a particular strategy with him.

Attempts to learn a foreign language were associated in both the qualitative and quantitative data with a positive disposition. Holly, Stephanie, and Linda all mention incorporating foreign language education in their classrooms, while Melody, on the opposite end of this spectrum, describes her position that students can keep this aspect of themselves hidden. In the quantitative data, teachers who report that they "somewhat" speak a foreign language, were also those associated with a more positive disposition as reported on the LATS score.

Increased experience teaching emergent bilingual students was also associated with positive dispositions. All participants of the study report a degree of trepidation when learning that they would be assigned an emergent bilingual student. However, all participants also report an increase in confidence after having worked with these students. Similarly, the survey suggests show that teachers who have worked with over 20 emergent bilingual students have a significantly more positive disposition toward emergent bilingual students than those who have worked with five or fewer.

Finally, according to the quantitative data, increased exposure to emergent bilingual students by way of professional development, is associated with more positive dispositions. This finding, however, was not supported in the qualitative data. Teacher participants, in general, had limited professional development in working with emergent bilingual students, although Holly and Jennifer mentioned that they would be interested in receiving more professional development in this area.

Interestingly, international experience, which I am defining as visiting a country that speaks a language other than English, is not associated with a more positive disposition toward 
emergent bilingual students. This is evident in both the qualitative and quantitative data. Melody, who has traveled to more countries than many of the other participants, is categorized as having the neutral/negative disposition. However, Susan who has a more positive disposition in the neutral/positive category, has only visited Toronto, Canada. Jennifer, in the same disposition category, has never been out of the United States. Therefore, increased international travel was not necessarily associated with a more positive disposition.

A teacher's perspective toward his or her role as a teacher and the willingness to accept obligation to teach all learners, may also influence his or her disposition toward the particular education needs of the linguistically diverse students in the classroom. Jennifer and Susan both approach the idea of teaching emergent bilingual students in the way they approach teaching all students. They are simply children, like any other, who have specific needs that must be addressed. Melody, however, believes that her role as a teacher is to "go in full force". She does not differentiate instruction as she does not want anyone to feel singled out. While she reports that this perspective comes from a place of care for her students, it does reduce the amount of instruction provided to students that meet their individual needs.

\section{Discussion for Research Question Two: What Resources and Strategies Do Teachers Draw Upon in Working with Their Emergent Bilingual Students?}

Personal resources are the most commonly used resources in both the qualitative and quantitative phases of data collection. Participants with the most experience in the qualitative research study reported using the most amount of personal resources and the least amount of conventional resources. Resource persons, empathy, and experience were the personal resources that were most commonly used. According to the quantitative results, personal resources were again reported to be used with the greatest frequency. 
Conventional resources are the least often used by teachers, according to the survey. Similar to the findings from the qualitative data, teachers with the most amount of teaching within the school district had close to the lowest use of conventional resources at 5.91. The lowest use of conventional resources was found, however in the group of teachers who report that they speak a foreign language fluently. Furthermore, the teachers of the qualitative phase of data collection report that due to district finances, conventional resources such as textbooks, paraprofessionals, and other such resources are lacking. These resources are designed to help teachers work with all students, not specifically emergent bilingual students. However, any resources that lighten the work of the teacher, frees them up to seek out the specific knowledge they may lack for working with their diverse classroom of students. One teacher, Susan, even mentioned that the lack of textbooks results in added work for her as she looked for their own classroom resources. She cited this work as the reason why she has not yet taken more time to look into the needs of her emergent bilingual students.

In both the qualitative and quantitative data, JGT has been found to be the most used category of pedagogical strategies. Teachers who have spent over 12 months in a country that speaks a language other than English and who speak a foreign language fluently are the most likely to use personal resources as well as LRT strategies. This may be because these teachers have more personal, first-hand language learning experience to draw upon in their teaching than teachers who have not had this experience. 


\section{Discussion for Research Question Three: How Does a Teacher's Disposition Inform the Resources and Strategies They Draw Upon in Working with Emergent Bilingual}

\section{Students?}

Both qualitative and quantitative data suggest that teachers' dispositions do, in fact, influence the resources and strategies that teachers use with emergent bilingual students. The linear regression analysis suggests that teachers with more positive dispositions will use more environmental and conventional resources as well as more JGT and LRT strategies.

The qualitative data provide insights into how the disposition influences these dependent variables. When Holly and Stephanie sought out their resource persons, they looked to individuals that have knowledge on how languages are learned, such as the school ESL teachers or friends, such as Christina, who has experience in teaching ESL German as a former high school German teacher. Stephanie mentioned that she knew this would be a good person to ask for help when she was assigned her first newcomer student because of Christina's specialized knowledge. Perhaps because of the resources they select, Holly and Stephanie both used more LRT strategies than the other teachers did in the study.

I had initially hypothesized that dispositions would potentially influence the degree to which resources and strategies are used and which resources and strategies are called upon. However, I also observed that the personal resource of experience impact disposition, resource use, and strategies separately. For example, Linda has a firsthand experience as a former emergent bilingual student. She does not seek out resource people who have the knowledge on how languages are learned and she does not select volunteers who can inform her on how to improve her practices for emergent bilingual students. Her repeated comparison of her own experiences to that of her students indicates that she uses her experience as her source of 
knowledge of how learning English should and should not be. She may feel that she therefore does not need to seek out help in other areas. She identifies that the ESL teacher brings specialized knowledge on languages, but does not turn to her. She also criticizes conventional resources, such as books and speaks with disdain regarding anything that would offer a scripted curriculum.

However, those participants who have international experience, but not firsthand experience as an emergent bilingual student were the participants who also sought out informed and experienced mentors. Stephanie chose an informed mentor. She then learned about strategies that are more effective for her students. Her success in the classroom then led to a positive disposition as she describes working with this population as the "highlight of [her] career." Similarly, Holly selected a mentor who was informed through his own personal experience. She often asks him for advice on what worked for him. However, unlike Linda, she is aware that her husband's experience may not completely reflect the experiences of her students.

\section{Discussion for Research Question Four: What is the Interplay Between the Teaching Strategies and Resources that Teachers Use in Working with Emergent Bilingual}

\section{Students?}

This question was the only research question of the four to that I analyzed solely through qualitative data. The data suggest that teachers who have close international experience as a personal resource were also those who were more likely to use LRT strategies.

Furthermore, resources and strategies interacted with one another. The resources a teacher turned to could result in different strategies that they used. For example, those who chose resource persons as mentors began to adopt more LRT strategies than those who did not. Strategies, such as getting to know students individually, could then become a resource that they 
turned to. However, teachers reflected some misunderstandings about their students' backgrounds, languages, cultures and present levels of English proficiency, as well as how the English language is learned and the ways that students will be asked to demonstrate their proficiency.

\section{General Conclusions and Summaries}

In this section, I will discuss the general conclusions that can be drawn from this study when the research questions and results are considered together. From this synthesis, a new model of teacher dispositions can be considered in light of the findings of this study and in relation to the Activity Theory framework. I will present this new model below and discuss the components of it while describing the general conclusions of the study.

\section{A New Model of Teacher Dispositions Through the Lens of Activity Theory}

Activity theory states that motivation leads to action (Lantolf, 2000). Motivations cannot be observed, only actions can (Lantolf, 2000). However different motivations can result in actions that are the same, but have different results (Lantolf, 2000).

Dispositions in the literature are often described on a continuum from negative to positive. The research in this field suggests a number of characteristics that tend to lead toward a more positive disposition of working with emergent bilingual students and situate the holder of the disposition along a continuum (see Figure 1). However, the qualitative data suggest that the dispositions of teachers are much more complex and interactive. There are myriad factors in a teacher's life that can be categorized as teacher beliefs and the teaching context, which may contribute to their disposition toward a variety of aspects of teaching an emergent bilingual student. Considering disposition on a single level limits the knowledge that can be gleaned from looking at how characteristics of dispositions influence one another and how they individually 
affect the way teachers view the many components that they must consider when educating an emergent bilingual student.

Rather than the continuum model, I would propose a model that is depicted in Figure 2 below based on the data gathered in this research.

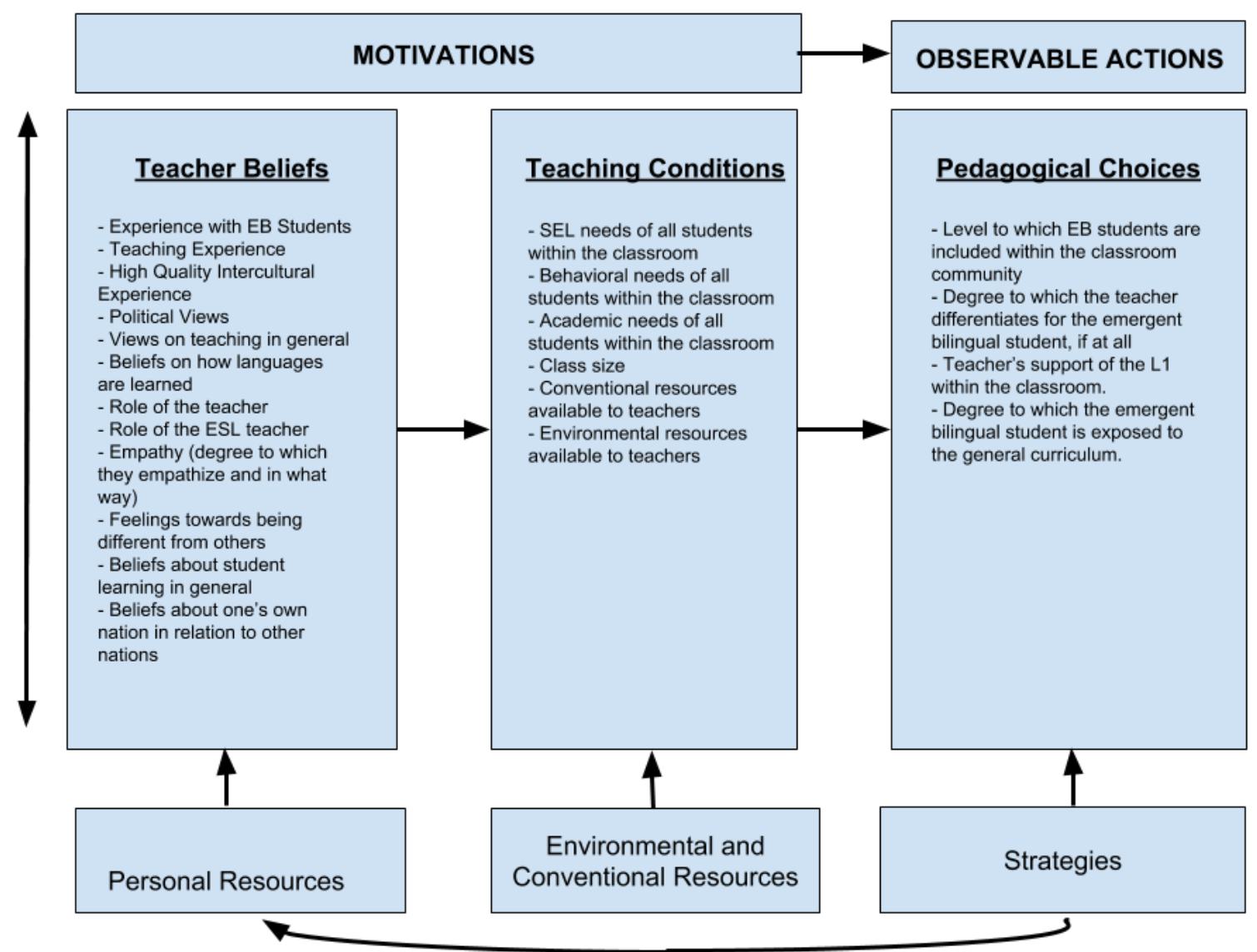

Figure 2: A new model of teacher dispositions.

Just as activity theory suggests, motivations influence actions (Lantolf, 2000). In the model presented above, the motivations are teacher beliefs as well as teaching conditions. The double-sided arrow to the left of the figure indicates that all the components of teacher beliefs can influence one another. This is also true for teaching conditions and pedagogical choices. 
Teacher beliefs must pass through a filter of the conditions in which the educator is teaching. From here, one can see the opportunities available to the teacher in terms of the resources he or she can turn to and the strategies that he or she can use. Activity theory tells us that it is only the actions that can be observed (Lantolf, 2000). Similarly, it is only the teachers' resource use and teaching strategies that one can observe. An action may be the same from one teacher to the next, but the motivation behind the action may be different, creating different results.

The examples of teacher beliefs, teaching conditions and pedagogical choices provided in the model were derived from the data collected and analyzed in this study. They are comprised of the codes gleaned from the qualitative data and come directly from teacher interviews, classroom observations, and the document analysis. Several of the concepts from each list were examined in more depth in the quantitative phase of data collection. Bilingual status of the teacher, the length of time a teacher spent in a country that speaks a language other than English, and the amount of training a teacher has had in working with emergent bilingual students have all been associated with teacher dispositions according to the quantitative data. However, other themes that were not explored, but were identified in the qualitative phase of data collection were also included in Figure 2. Similarly, dispositions were all associated with the use of the environmental and conventional resources and also the use of JGT and LRT strategies. The examples of teacher beliefs, teaching conditions, and pedagogical choices provided in this model are not intended to be an exhaustive list. Rather, they are listed to illustrate the concepts of the new model for teacher dispositions that were identified in the qualitative phase of data collection. Meanwhile, the arrows of the model demonstrate a directionality that was identified in both the qualitative and quantitative phases. 
In the development of this new model, I considered several questions. To begin with, I needed to consider in what way activity theory was applicable to the data. I was able to glean motivation from the interviews and pedagogical choices could be ascertained from the observations. However, in my data collection, I was surprised how much teaching conditions continued to be discussed by teachers in the interviews as they discussed their work with a small population of their students. It was apparent that these were resources that teachers either had or lacked and they played a strong role in educational decision-making for all students.

Secondly, it became evident that the consideration of teacher disposition towards emergent bilingual students along a continuum was not sufficient to accurately portray this complex construct. Instead, there are a variety of interactive factors at play. Furthermore, teacher dispositions are likely not static, but dynamic and continuously changing. While one may be able to identify a teacher's disposition as more negative or more positive, one should bear in mind that these dispositions are fluid and that the context of the teaching environment will interact with dispositions to result in the observable teaching practices.

The new model for teacher dispositions was the result of the consideration of the two above-stated questions. I will provide an example of three teachers who all include a child in a general education math lesson, which is the observable action. In this example, I use a female pronoun for the teachers and a male pronoun for the student. The first teacher chooses to include the student because she has a belief that it is a negative thing to be different and does not want the child to feel singled out (motivation). One observing this math lesson would see the emergent bilingual student receive instruction all in English with no learning materials or lesson features that provides linguistic differentiation. A second teacher may choose to include the child in the math lesson because she believes that all students should have access to the general curriculum at 
a level that meets that child's needs (motivation). This lesson may have many differentiated features, such as extra-linguistic supports and peer tutors and the students are provided with learning materials that meet his or her linguistic needs. Still a third teacher may also believe that all students should have access to the general curriculum, but at the same time hold a belief that immersion provides the best method by which students can learn English (motivation). Therefore, the lesson may include differentiation such as extra-linguistic supports, but not peer tutors. The work the child is asked to complete may be differentiated by skill level, but may not provide the linguistic supports the second teacher in the example provided.

Teacher beliefs. The beliefs of the teachers are the personal resources that teachers carry with them. These are developed over time through experiences such as years teaching, intercultural experiences, personal stories of their own school years, beliefs about how students learn, and their views on their role as the teacher or the students' roles as students.

International/intercultural experience. Intercultural experience is one factor that may be grouped under "Teacher Beliefs" in the model above. While international experiences, that is visiting another country, can be formative, I would argue that the quality of that experience (living with someone from a different culture, such as Holly) can be more impactful than other types of intercultural experiences (e.g. traveling as a tourist with an American friend). The quantitative results only consider an international experience by asking if the participant has visited a country that speaks a language other than English. The survey does not look at other intercultural experiences. The study conducted by Stanoscheck Youngs and Youngs, Jr. (2001) considered intercultural experience on a variety of levels. These included if the teacher had "traveled abroad, lived abroad, taught abroad, and hosted a foreign student" (p. 113). They found significant results for teachers who had lived abroad and taught abroad, but, just as in this study, 
not for teachers who had only traveled abroad. However, they noted that teachers who had hosted a foreign student did not indicate a significant relationship.

The qualitative data of this study suggests that hosting a foreign student may have a bigger impact than was observed in the Stanoscheck Youngs and Youngs, Jr. (2001) study. It also had a bigger impact than those who reported that they had traveled to a country that speaks a language other than English. Social Contact theory developed by Allport (1954) may explain this phenomenon. In this work, Allport (1954) describes the groups to which individuals are affiliated as in-groups. These in-groups are, in many cases are considered to be "good" by the individual because they are given to that person and they come from that child's family (p. 29). The individual often feels a sense of loyalty to their in-groups (Allport, 1954).

Pettigrew, Tropp, Wagner, and Crist (2011) conducted a meta-analysis of 515 studies that examine intergroup contact and prejudice. They discovered that "samples that were rated as having most of Allport's optimal conditions have significantly greater prejudice reduction effects $(-.29)$ than those without these conditions" (p. 275) such that as optimal contact increased, prejudice decreased (Pettigrew, et al., 2011). Allport's optimal contact conditions include “equal status, common goals, no intergroup competition, and authority sanction" (Allport, 1954). Furthermore, these authors noted the importance of friendships between groups in reducing prejudice. They stated, "Friendship invokes many of the optimal conditions for positive contact effects: it typically involves cooperation and common goals as well as repeated equal-status contact over an extended period and across varied settings" (pp. 275-276). This point explains why Holly's experience with her foreign exchange student was transformative for her more so than the travels of the other teacher participants. 
While international experience may lead to positive dispositions, if one experiences travel as a visitor going to tourist destinations with other foreigners and having limited interaction with the people of the country they are visiting, the impact on the teacher's conception of culture may only be marginally impacted. Take for example someone who has an immersion experience as a volunteer worker in Mexico City for one week compared to someone who went to Cancun for Spring Break for one week. Both participants would be able to report that they have visited a country that speaks a language other than English. However, the impact of their visit may be very different.

Furthermore, travel in this surface-level manner may even reinforce negative stereotypes about cultures outside of the one's own in-group. Melody's international experience was in more of a tourist role than in a high contact, immersive visit. Rather than visiting and staying with friends, as Susan did in her trip to Canada or Holly did in her visit to Denmark, Melody stayed in hotels with other Americans. In reporting her travel experience, she described feeling fear because of an American movie she had seen prior to the trip, used mockery to describe the Italian fire department, and finally, she cites only limited cultural information as what she "learned most" from her visit to Rome. While it is beyond the scope of this study to investigate if Melody did experience any positive results from her travels, the research suggest that had she had more of an immersive experience, she would have had more lasting and impactful effects.

Linda's life in Vietnam, where she was born, was certainly more immersive experience than a visit to a foreign country as this was her home for the first 12 years of her life. However, while in Vietnam, she and her family felt discrimination and, in her description, was run out of the country due to her ethnic background. While I did not explore her personal opinion of the Vietnamese, she indicated that her mother maintained a negative opinion of their experiences. 
She states, "If I told my mom that I wanted to go back and visit Vietnam, she would..." She trailed off and made a look of fear of how her mother might react to if she would ever state a desire to return to Vietnam. Pettigrew, et al. (2011) wrote "not all intergroup contact reduces prejudice" (p. 277). Negative results occur in environments in which a threat is present (as with Linda's mother) and the individuals do not choose to encounter one another.

Empathy. Empathy represents another frequently cited component of personal resources that teachers turn to for developing their approach to emergent bilingual students. Therefore, empathy is a component of the teacher beliefs category. While personal experience, including intercultural experience, may bring a lot of empathy, in the qualitative data it also led to assumptions by teachers that their students' experiences will be the same as their experiences had been or the experiences of their loved ones. However, different children have different immigration experiences. Teachers must recognize that while their personal knowledge and experience is valuable, they cannot assume that what worked for them or their loved ones will work for all of their students. It does not trump getting to know their students individually and considering what will work for their individual needs.

Empathy is often used by teachers as a resource. They empathize with the students or at least sympathize with their students and that informs their decision-making. However, lacking additional information that could be provided by specific knowledge on working with emergent bilingual students, they make assumptions that are not necessarily useful. For example, Melody empathizes with her students and expresses this by discouraging her students from standing out. She believes that students do not want to speak their primary language in school. This is a harmful practice and many are proud of their L1 and country of origin. Research suggests that this practice could lead to language shaming, which results in disconnect within families. 
Research by Winstead and Wang (2017) examined the area of language shaming. The participants of their case study included eight bilingual Spanish-English teachers who were asked about their own perceptions of native language use from their childhoods as they grew into adults and also these perceptions may influence their teaching practices. Winstead and Wang (2017) wrote:

While growing up as bilinguals there was shame in being deficient in English associated with their use of Spanish language, code-switching, and thick Spanish accents. All of them related how deficit discourses had affected their own schooling, and that they had been restricted and shamed from using their native language with newcomers in schools in present-day circumstances. (p. 22)

Winstead and Wang noted that a loss in their L1 and their cultural identity was the result of the language shaming they experienced as children and that they continued to experience as teachers. In this way, Melody's empathy leads to practices that may be harmful to her students.

The immersion myth. Despite research in the area of how children learn languages that refute the "sink or swim" philosophy behind language immersion (Berliner \& Glass, 2014), general education teachers still maintain the idea that providing English-only instruction will help students learn English and content faster. Although Stephanie had access to resources in a child's home language, Stephanie said that she knows that is not what the child needs in order to learn English and therefore withheld these resources from her newcomer students. In this fashion, regardless of the veracity of the belief, a teacher's perception of how language is learned can impact the teaching strategies that they employ.

Teacher mentoring. Analyses of the qualitative data suggests that teachers often feel apprehensive about working with emergent bilingual students for the first time. This is especially 
true for teachers who have been assigned a newcomer student and for teachers who have fewer years of experience in the district and therefore fewer personal resources to draw upon. Several teachers describe searching out individuals who may be able to volunteer with that student or who can provide insight into instructing that student. Teachers do not report turning to the school ESL teacher to the same degree as they turn to their self-selected resource persons. Given that the teachers are seeking out these mentors themselves, it makes sense that they find someone with whom they first have a friendly and positive relationship and only secondarily look for someone with specialized knowledge in this field. Portner (2008) stated, "Mentoring requires interaction. But in order to be productive, mentor-mentee interactions must take place within a relationship that includes mutual trust, honesty, respect, and a joyful willingness to work together" (p. 12). When self-selecting a mentor, the teachers look first to someone with whom that relationship is established.

These self-selected resource persons have a strong impact in the pedagogical perspectives of teachers and the strategies that the teachers employ. This is true not only for the participants of this study, but also for people in many professions. Alper (2017) contended that mentors can make dramatic impacts on children and adults in various fields. Therefore, it is essential that the teachers have mentors available to them who can provide high quality guidance.

Resource distribution. Many of the participants I interviewed noted that there is a challenge presented to them in the lack of conventional resources by the district due to financial limitations, such as textbooks, or environmental resources, such as paraprofessionals. However, during observations, it was apparent that the lack of resources provided by the school district may have had an uneven result. Teachers located in school A were either observed to have or the teacher reported having a significant number of students affected by emotional concerns. 
Throughout observations, teachers in school A were observed using student independent work time to resolve disagreements, offer hugs and comfort, or deal with discipline issues. In schools $\mathrm{B}, \mathrm{C}$, and D, teachers used independent work time to check in with emergent bilingual students as well as others or pull students into small group for further support.

Application of Positioning Theory. Positioning theory and activity theory were the combined theoretical frameworks of this study. It is important to recognize the way that teacher positioning plays a role in this activity theory-based model presented above. Positioning describes the way that identities are constructed through social contexts, which occurs discursively (Harre \& van Lagenhove, 1999). This is a continuous and evolving process. Because I did not examine the interactions between students and teachers in this study, I can only consider positioning in the way that teachers describe themselves and their students. Positioning occurs through position, acts, and story lines (Harre \& Moghaddam, 2003). Story lines represent an interpretation about a pattern of acts. Positioning theory was examined in terms of the stories that the teachers told themselves and shared with me about working with emergent bilingual students.

Each teacher had their story lines that played a role in how they approached their emergent bilingual students. Some of these story lines were present before the teacher met the emergent bilingual student. For example, Holly describes herself as “obsessed with culture.” In this way, the story she tells herself is that interacting with new cultures is fun and she personally has much to gain from this. Some teachers developed their story line through interaction with emergent bilingual students. Stephanie would be an example of this. She was nervous about having her first emergent bilingual student and turned to a friend and former colleague for help. Through the adoption of effective strategies, Stephanie became more confident in her work with 
emergent bilingual students and her story line now states that this is the "highlight of [her] career."

Melody appears to have an ethnocentric story line. This was evident in her description of her visit to Italy as well as in the way approached the differences present in her emergent bilingual students compared to the American students. Her story line is that being different is bad.

Linda's story line is based on her personal experience of learning English. She states that she had to work very hard to learn both English and content. She also compares her experience as an emergent bilingual student to that of her students and has come to the conclusion that for a variety of reasons, such as the ability for parents to be involved and the responsibilities and expectations of her students at home, that her students are different. However, she also believes that having a global perspective is valuable and in this way encourages her emergent bilingual students to share their cultures with the Americans in her class. Her story line also involves the loneliness she felt as a newcomer student herself. In this way, she positions her students as similar to herself and does her best to help them feel included.

These story lines then play a role in the way the teachers consider their emergent bilingual students and what they bring to the classroom. They may also impact the way a teacher approaches his or her teaching conditions. These factors together are the motivations that will later impact the strategies that teachers use within their classrooms.

Teaching conditions. Teaching conditions are the conventional and environmental resources available to the teacher. Conditions are up of the enrollment size of the classroom, the social and emotional, behavioral, or academic knowledge or needs of the entire population of 
students, the conventional resources available to the teacher, and the support that the teacher has from the building principal, ESL teacher, or other building or district personnel.

In this study, teachers with greater needs in terms of student behavior or social and emotional development were observed to have fewer pedagogical choices open to them as their classroom time was more often dictated by a need to help process emotional struggles of students.

Susan and Stephanie also reported that the lack of textbooks and the limited number of paraprofessionals were a source of stress. They stated that this lack of resources takes away the teachers' time to devote to addressing the needs of their emergent bilingual students. Again, the environmental condition can result in a different set of available resources from which to choose and becomes a condition that affects their teaching strategies.

The research in this area suggests that teaching conditions that have fewer conventional resources can result in teacher attrition (Harrell, Leavell, van Tessel, \& McKee, 2004). According to Shirrell and Reininger (2017) teacher working conditions can:

Encompass a variety of aspects of schools, including their physical environments; organizational patterns of authority, supervision, and interaction among employees; employees' characteristics, roles, and statuses; the sense of equity and voice among the staff; the strength and supportiveness of the school culture; teachers' opportunities for learning and growth; and educational aspects of schools such as curricula and assessments. (p. 52)

Teaching conditions can also include class size, teaching load, and appropriate curriculum, resources, and materials (Johnson, 2006). While an argument can be made that teachers with 
more stress are less effective (Wong, Ruble, Yu, \& McGrew, 2017), teachers working in poorer working conditions simply do not have as many pedagogical choices available to them.

Pedagogical choices. This category in the model is comprised of the actual resources that teachers use and the strategies that they employ. This category, which is the only one that can be observed, represents the degree to which the emergent bilingual student is included within the classroom, the degree to which the teacher differentiates, the level of the use of the child's L1, and other such pedagogical decisions teachers make each day.

Knowledge of students. Many teachers reported getting to know students was a strategy that they use and they describe this knowledge as a resource in making pedagogical decisions. It is important, therefore that teachers have correct information about these students. Several teachers reported what appeared to be incorrect information about their students' backgrounds, proficiency levels, understanding of the ACCESS test, and knowledge of the child's home country, culture, and language. They need to know their proficiency levels and teach accordingly. They also need to know their home countries, languages, and status within the ESL program. According to Lucas et al. (2008):

Though ELLs tend to be discussed as if they were a homogenous group, they are not. They enter the US schools with varying levels of oral proficiency and literacy (in both English and their native language) as well as prior knowledge of and experiences with subject matter (p. 366).

Teachers need to get to know their emergent bilingual students, just as they get to know all students, so they can capitalize on the funds of knowledge that they bring to their classroom. Supporting the background of their emergent bilingual students leads to more effective teaching strategies as well as supporting an additive biculturalism and bilingualism (deJong, 2013). 
However, learning about the lives of the emergent bilingual students needs to be done in a manner that also recognizes the individuality of the child. Villegas and Lucas (2007) wrote:

We are not suggesting that teachers learn generic information about specific cultural or social groups. Such thinking leads to stereotypes that do not apply to individual students. Instead, teachers need to know something about their students' family makeup, immigration history, favorite activities, concerns, and strengths. Teachers should also be aware of their students' perceptions of the value of school knowledge, their experiences with the different subject matters in their everyday settings, and their prior knowledge of and experience with specific topics in the curriculum. (p. 31)

Villegas and Lucas (2007) go on to state, "To make productive use of this information, teachers must possess two fundamental qualities: They must have sociocultural consciousness and hold affirming views of diversity" (p. 32). While the teachers in this study attempted to learn about their students, there were many misunderstandings that resulted in ineffective practices and prevented them from adopting linguistically responsive practices, such as not knowing which country a child came from or which language he or she speaks.

Access to the curriculum. Finally, although teachers are required to follow the stateadopted standards and the school district mandates, they still act as the gatekeepers to the curriculum. They are deciding what aspects of the curriculum the child will be exposed to, to what degree, and who will provide that instruction. In many cases, the teachers may know their students' abilities and can appropriately make this choice. However, Linda maintains that her student cannot participate in certain subjects. She has therefore made the decision to send the child out of the room with the purpose of providing him instruction at his level. This is a decision she believes she is making out of love. She believes she is providing him with an academic 
foundation so that he can be prepared for more challenging math and reading tasks. However, the result is that is missing out the general curriculum. Rather than being asked to participate in the fourth grade math curriculum, he is doing the remedial work of addition, subtraction, multiplication, and division facts. Rather than considering ways to make the math lesson more tangible for her newcomer student to receive, she instead made a decision that he will not receive that instruction.

The child's access to the curriculum has long been a topic that is relevant to special education contexts (Skiba et al., 2008). Building from the civil rights movement, special education advocates have had to fight for equal access to the curriculum (Skiba, et al., 2008), which continues to be an ongoing struggle (Mason-Williams, 2015). It is apparent from this research that there is a need for all students to receive equal access to education, including emergent bilingual students. Darling-Hammond (2015) outlined the present situation of the unequal access to education in this way:

In this respect, too, separate schools and tracks undermine democracy by segregating students by race, language, and social class, and by encouraging silence and separation where communication and connections are needed. These practices heighten divisions among groups and prevent many young people from becoming active social participants in the life of their school-and later in the broader community where, ultimately, we must all learn to work and live together. (p. 62)

This is a great concern for teachers who have positive dispositions toward emergent bilingual students. However, giving a teacher who adheres to deficit thinking the power to decide how much of the curriculum his or her emergent bilingual student is exposed to, can ultimately lead to oppression. A belief that the child naturally has a lower educability or reduced motivation to 
learn, may lead to lower expectations, social and educational segregation, even when officially the child is placed in the classroom with native English-speaking peers, as was the case with Linda's students.

\section{Implications}

With this study in mind, school districts need to ensure that teachers of emergent bilingual students, particularly those with limited experience with this population have enough support available to them. This support can come in the form of access to conventional resources and the assignment of a knowledgeable mentor. Each building in this district is assigned an ESL teacher. The ESL teacher is required to provide professional development. ESL teachers should be provided with enough time in their schedules to offer mentoring to these teachers.

Additional resources need to be provided to the schools that have greater behavior and SEL needs. Providing this additional support will offer the ability to provide more individualized instruction for emergent bilingual students. Schools that struggle with behavior and social emotional learning need counselors and other support personnel on hand to help students develop in this area, thereby giving teachers the time they need to give extra supports to students in their classrooms who may need it.

The school district needs to be clear about the importance of giving equal access to education for all students. Teachers in this study made curricular decisions for emergent bilingual students. However, this meant different things for different students placed in different buildings and classrooms. A clear delineation of the teacher's role, ESL teacher's role, and student's role would be helpful in articulating this vision and its implementation for teachers.

Many states are moving toward a push-in model for ESL services in which the ESL teacher enters the general education classroom to provide English language instruction for the 
emergent bilingual student. In the ideal scenario, the ESL teacher would be viewed as a resource person to the general classroom teacher and would provide a way to ensure that the emergent bilingual child is receiving access to the same curriculum that the other students receive. However, this again would require a clear definition of roles for the classroom teacher and ESL teacher that the qualitative research suggested was missing. It also requires the ESL teacher to present himself or herself as a knowledgeable professional in this field.

All teachers need to regularly consider their biases. It would be useful for schools to conduct bias training in new teacher orientation sessions and start each year with a discussion in this area. The existing biases of individuals present an argument that school districts should do their best to recruit and retain teachers who reflect the backgrounds of the students in the schools.

\section{Implications for Professional Development and Teacher Preparation Programs}

Teachers need to move away from the idea that effective teaching for emergent bilingual students is JGT (de Jong and Harper, 2005). The professional development that pre-service and in-service teachers receive should highlight both the JGT practices that are helpful for emergent bilingual students, but also LRT strategies.

Pre-service teaching programs should incorporate MSL programs. This may be most effective when it is emphasized that the teacher is to learn from the multicultural communities in which they find themselves as much as they are to teach and serve those communities. The more exposure and contact these pre-service teachers have with diverse populations, the more likely it is that they will have positive dispositions towards these populations. 


\section{Limitations}

There are several limitations to this study that need to be identified. To begin with the teacher surveys and interviews may be inaccurate as they are self-reported. It is possible that the dispositions that teachers presented during interviews were more complex than what they shared. It is also possible that the survey did not capture the full depth and breadth of the teacher's dispositions. Furthermore, the response rate from the online survey was at $18.5 \%$. This is a relatively low response rate.

Additionally, both Holly and Susan work in the same building and were found to have quite positive dispositions toward their emergent bilingual students and students in general. Both Melody and Jennifer work in the same building and are also identified as having a type of neutral disposition. In this study I did not look at the role that the school culture played on the dispositions of the teachers, however, it is possible that schools can have an impact on the attitudes of teachers toward their students. There may be other factors at play that were not explored in this present study.

\section{Recommendations for Future Research}

Now that a new model of teacher dispositions toward emergent bilingual students has been developed, the model needs to be empirically tested. Future studies can examine the interaction of these beliefs on one another, as well as the teaching conditions and strategies. One can also examine the way that each of these components impact each of the teaching strategies individually.

Teaching conditions was not an area that was explored as a part of the research questions for this study. However, the topic of teaching conditions came out organically in the interviews. This is an area that needs to be examined to a greater degree. 
Another topic that emerged from the teacher interviews was the way that their beliefs on how languages are learned impact their strategies. I would like to consider how these beliefs developed and what the exact impact is on their teaching. This can be taken to examine student language proficiency as related to these teacher beliefs.

Finally, despite having a mandated curriculum following state-adopted standards, not all students were receiving this education. Future studies need to look into the degree to which emergent bilingual students, particularly newcomer emergent bilingual students have access to the general curriculum. In this research, one can examine what the effect of access, or lack of access, to this curriculum is over time. 


\section{REFERENCES}

Albert Shanker Institute. (2016). A look at teacher diversity. AmAppenderican Educator, 40(3), 18-19.

Allport, G. W. (1954). The nature of prejudice. Reading, MA: Addison-Wesley.

Alper, P. (2017). Teach to work: How a mentor, a mentee, and a project can close the skill gap in America. New York, NY: Bibliomotion.

Andrei, E., Ellerbe, M., \& Cherner, T. (2015). 'The text opened my eyes': A book club on teaching writing to ELLs. The Electronic Journal for English as a Second Language, 19(3), 1-22.

Anderson, K. T. (2009). Applying positioning theory to the analysis of classroom interactions: Mediating micro-identities, macro-kinds, and ideologies of knowing. Linguistics and Education, 20, 291-310.

August, D. and Shanahan, T., eds. 2006. Developing Literacy in Second-Language Learners: Report of the National Literacy Panel on Language-Minority Children and Youth. Mahwah, NJ: Lawrence Erlbaum;

Berger, R. (2015). Now I see it, now I don't: Researcher's position and reflexivity in qualitative research. Qualitative Researcher, 15(2), 219 - 234.

Berk, L.E. \& Meyers, A.B. (2016). Infants and children: Prenatal through middle childhood $\left(8^{\text {th }}\right.$ ed.). Boston, MA: Pearson.

Berliner, D. C., \& Glass, G. V. (2014). 50 myths and lies that threaten America's public schools. New York, NY: Teachers College.

Brinkmann, S., \& Kvale, S. (2015). InterViews: Learning the craft of qualitative research interviewing ( $3^{\text {rd }}$ ed.). Thousand Oaks, CA: SAGE. 
Brooks, K., \& Karanthos, K. A. (2009). Building on the cultural and linguistic capital of English learner (EL) students. Multicultural Education, 16(4), 47-51.

Byrnes, D. A., Kiger, G., \& Manning, L. (1993, April). Teacher attitudes about language differences. Paper presented at the Association for Childhood Education International Study Conference, Phoenix, AZ.

Byrnes, D. A., \& Kiger, G. (1994). Language Attitudes of Teachers Scale (LATS). Educational and Psychological Measurement, 54(1), 227-231.

Byrnes, D. A., Kiger, G. \& Manning, M. L. (1998). Classroom teachers and language minority students. Educational Research Quarterly, 22, 26-32.

Borko, H. (2004). Professional development and teacher learning: Mapping the terrain. Educational Researcher, 33(8), 3-15.

Boughton, C. (2015). Boom, bust, exodus: The rust belt, the maquilas, and a tale of two cities. New York, NY: Oxford University Press.

Carroll, J. (2006). "Roughly 1 in 10 Americans Have Religious Objections to Halloween." Gallup.com Gallup, Inc, Retrieved from: https://news.gallup.com/poll/25282/roughlyamericans-religious-objections-halloween.aspx

Caesar, L. G., \& Nelson, N. W. (2014). Parental involvement in language and literacy acquisition: A bilingual journaling approach. Child Language Teaching and Therapy, 30(3), 317-336.

Chang, S., Anagnostopoulos, D., \& Omae, H. (2011). The multidimensionality of multicultural service learning: The variable effects of social identity, context and pedagogy on preservice teachers' learning. Teaching and Teacher Education, 27, 1078-1089. 
Chenail, R. J. (2011). Interviewing the investigator: Strategies for addressing instrumentation and researcher bias concerns in qualitative research. The Qualitative Report, 16(1), 255262.

Cheatham, G., Jimenez-Silva, M., Woodrich, D. L., \& Kasai, M. (2014). Disclosure of information about English proficiency: pre-service teachers' presumptions about English language learners. Journal of Teacher Education, 65(1), 53-62.

Clark, U. (2013). A sense of place: Variation, linguistic hegemony and the teaching of literacy in English. English Teaching: Practice and Critique, 12(2), 58-75.

Coady, M., Harper, C., \& de Jong, E. (2011). From pre-service to practice: Mainstream elementary teacher beliefs of preparation and efficacy with English language learners in the state of Florida. Bilingual Research Journal, 34, 223-239.

Cohen, D. K., Raudenbush, S. W., \& Ball, D. L. (2003). Resources, instruction, and research. Educational Evaluation and Policy Analysis, 25(2), 119-142.

Common Core State Standards Initiative (2019). English Language Arts Standards. Retrieved from http://www.corestandards.org/ELA-Literacy/

Conner, J.O. Learning to unlearn: How a service-learning project can help teacher candidates to reframe urban students. Teacher and Teacher Education, 26, 1170-1177.

Corbin, J. \& Strauss, A. (2015). Basics of qualitative research: Techniques and procedures for developing grounded theory ( $4^{\text {th }}$ ed.). Thousand Oaks, CA: SAGE.

Corbin Dwyer, S. \& Buckle, J. L. (2012). The Space Between: On Being an Insider-Outsider in Qualitative Research. International Journal of Qualitative Method, 8(1), 54 - 63.

Creswell, J. W., \& Plano Clark, L. (2011). Designing and conducting mixed methods research. Thousand Oaks, CA: SAGE. 
Cubit Planning, Inc. (n.d.). Illinois demographics. Retrieved from www.Illinoisdemographics.com

Cummins, J. (2012). The intersection of cognitive and sociocultural factors in the development of reading comprehension among immigrant students. Reading and Writing: An Interdisciplinary Journal, 25(8), 1973-1990.

Cummins, J. (1991). Interdependence of first and second-language proficiency in bilingual children. In E. Balystock (Ed.), Language processing in bilingual children. (pp. 70-89). Cambridge, England: Cambridge University Press.

Darling-Hammond, L. (2015). The flat world and education: How America's commitment to equity will determine our future. New York, NY: Teachers College Press.

Davies, B., \& Harré, R. (1990). Positioning: The discursive production of selves. Journal for the Theory of Social Behavior, 20(1), 43-63.

deJong, E.J. \& Harper, C.A. (2005). Preparing mainstream teachers for English-language learners: Is being a good teacher good enough? Teacher Education Quarterly, 101-124. deJong, E. (2013). Preparing mainstream teachers for multilingual classrooms. Association of Mexican-American Educators, Special Invited Issue, 7(2), 40-49.

deJong, E. \& Harper, C. (2013). ESL is good teaching "Plus": Preparing standard curriculum teachers for all learners. In M. E. Brisk (Eds.), Language, Culture, and Community in Teacher Education (pp. 127-157). Mahwah, NJ: Lawrence Erlbaum.

Delgado Bernal, D. (2002). Critical race theory, Latino critical theory, and critical racedgendered epistemologies: Recognizing students of color as holders and creators of knowledge. Qualitative Inquiry, 8(1), 105-126. 
DelliCarpini, M. \& Alonso, O.B. (2014). Teacher education that works: Preparing secondary level math and science teachers for success with English language learners through content based instruction. Global Education Review, 1(4), 155-178.

de Oliveira, L. C. (2011). In their shoes: Teachers experience the needs of English language learners through a math simulation. Multicultural Education, 19(1), 59-62.

disposition. (2017). In Merriam-Webster.com. Retrieved from https://www.merriam-webster.com/dictionary/disposition

Doabler, C. T., Nelson-Walker, N., Kosty, D., Baker, S. K., Smolkowski, K., \& Fien, H. (2013). Explicit instructional interactions: Observed stability and predictive validity during early literacy and beginning mathematics instruction. Paper presented at the Society for Research on Educational Effectiveness, Washington, D.C. Abstract retrieved from https://files.eric.ed.gov/fulltext/ED563121.pdf

Dominguez, H. (2011). Using what matters to students in bilingual mathematics problems. Educational Studies in Mathematics, 76(3), 305-328.

Dulay, H. and Burt, M. (1977) Remarks on creativity in language acquisition. In M. Burt, H. Dulay and M. Finnochiaro (Eds.) Viewpoints on English as a second language, (pp. 95126) New York, NY: Regents.

Echevarria, J., Vogt, M., \& Short, D. (2017). Making content comprehensible for English learners: The SIOP® model ( $5^{\text {th }}$ ed). Boston, MA: Pearson.

Fernandez, N., \& Inserra, A. (2013). Disproportionate classification of ESL students in U.S. special education. TESL-EJ, 17(2), 1-22. 
Filmore, L. W. \& Snow, C. E. (2002). What teachers need to know about language. In C. T. Adger, C. E. Snow, \& D. Christian (Eds.), What teachers need to know about language (pp. 7 - 55). McHenry, IL: Center for Applied Linguistics.

Franquiz, M. E., del Carmen Salazar, M., \& DeNicolo, C. P. (2011). Challenging majoritarian tales: Portraits of bilingual teachers deconstructing deficit views of bilingual learners. Bilingual Research Journal, 34(3), 279-300.

Gates, G. S., \& Lichtenberg, K. (2005). Accountability data and decision making in Texas bilingual education programs. Journal of Latinos and Education, 4(4), 271-282.

Garcia, O. (2009). Emergent bilinguals and TESOL: What's in a name? TESOL Quarterly: A Journal for Teachers of English to Speakers of Other Languages and of Standard English as a Second Dialect, 43(2), 322-326.

Gay, G. (2002). Preparing for culturally responsive teaching. Journal of Teacher Education, 53(2), 106-116.

Genesee, F., Lindholm-Leary, K., Saunders, W., and Christian, D. 2006. Educating English Language Learners. New York: Cambridge University Press.

Gladson-Billings, G. (1995). Toward a theory of culturally relevant pedagogy. American Educational Research Journal, 32(3), 465-491.

Goldenberg, C. (2008). Teaching English language learners: What the research does- and does not- say. American Educator, 32(2), 8-44.

Guillemin, M. \& Gllam, L. (2004). Ethics, reflexivity and 'ethically important moments' in research. Qualitative Inquiry, 10, $261-280$.

Harding, S. (1987). The method question. Hypatia, 2(3), 19-35. 
Harré, R. \& Moghaddam, F. M. (2003). Introduction: the self and others in traditional psychology and in positioning theory. In R. Harré and F. M. Moghaddam (Eds.) The self and others: Positioning individuals and groups in personal, political, and cultural contexts (pp. 1-12). Westport, CT: Greenwood Publishing Group.

Harré R. \& van Langenhove, L. (1999). The dynamics of social episodes. In R. Harré \& L. van Langenhove (Eds.), Positioning theory: Moral contexts of intentional action (pp. 1-13). Malden, MA: Blackwell Publishers.

Heineke, A.J. (2014). Dialoguing about English learners: Preparing teachers through culturally relevant literature circles. Action in Teacher Education, 36(2), 117-140.

Hertzog, L. (2011). Can a successful ESL teacher hold deficit beliefs of her students' home languages and cultures? Multicultural Perspectives, 13(4), 197-204.

Hutchinson, M. (2013). Bridging the gap: pre-service teachers and their knowledge of working with English language learners. TESOL Journal 4(1), 25-54.

Illinois State Board of Education (2017) Illinois report card. Retrieved from https://www.illinoisreportcard.com/District.aspx?districtid=33048205026

Illinois State Board of Education (2019). English Learners: English Language Proficiency Assessments. Retrieved from https://www.isbe.net/Pages/ACCESS-for-ELLs.aspx

Jarrett, D. (1999). The inclusive classroom: Teaching mathematics and science to English language learners. Portland, OR: Northwest Regional Educational Laboratory. Retrieved from https://educationnorthwest.org/sites/default/files/11.99.pdf

Jensen, D. (2008). Access. In L. Given (Ed.), SAGE encyclopedia of qualitative research methods. (vol. 2, pp. 2-3). Thousand Oaks, CA: SAGE. 
Jiménez, R. T., David, S., Pacheco, M., Risko, V. J., Pray, L., Fagan, K., \& Gonzales, M. (2015). Supporting teachers of English learners by leveraging students' linguistic strengths. Reading Teacher, 68(6), 406-412.

Johnson, S. M. (2006). The workplace matters: Teacher quality, retention and effectiveness. Washington, DC: National Education Association. Retrieved from http://www.nea.org/assets/docs/HE/mf_wcreport.pdf

Jussim, L., \& Harber, K.D. (2005). Teacher expectations and self-fulfilling prophecies: Knowns and unknowns, resolved and unresolved controversies. Personality and Social Psychology Review, 9(2), 131-155.

Kincheloe, J. L., McLaren, P., \& Steinberg, S. R. (2011). Critical pedagogy and qualitative research: Moving to the bricolage. In N. Denzin \& Y. Lincoln, The Sage handbook of qualitative research ( $4^{\text {th }}$ ed., 163 - 178). Thousand Oaks, CA: SAGE.

Krashen, Stephen D. 1982. Principles and practice in second language acquisition. Oxford: Pergamon. http://www.sdkrashen.com/Principles_and_Practice/Principles_and_Practice.pdf.

Krashen, S. (2013). Does SIOP research support SIOP claims? International Journal of Foreign Language Teaching, 8(1), 11-24.

Lambert, R., Boyle, L., Fitchett, P., \& McCarthy, C. (2017). Risk for occupational stress among U.S. kindergarten teachers. Journal of Applied Developmental Psychology. https://doi.org/10.1016/j.appdev.2018.07.003

Lantolf, J. P. (2000). Introducing sociocultural theory. In J. P. Lantold (Ed.), Sociocultural theory and second language learning (pp. 1-26). New York, NY: Oxford University Press. 
Lim, M. (2012). Unpacking parent involvement: Korean American parents' collective networking. School Community Journal, 22(1), 89 - 109.

Lin, S. M. (2015). A study of ELL students' writing difficulties: A call for culturally, linguistically, and psychologically responsive teaching. College Student Journal, 49(2), 237-250.

Lincoln, Y.S. \& Guba, E.G. (1985). Naturalistic inquiry. Newbury Park, CA: SAGE.

Lopez Leiva, C. A., Torres, Z., \& Khisty, L. L. (2013). Acknowledging Spanish and English resources during mathematical reasoning. Cultural Studies of Science Education, 8(4), 919-934.

Lucas, T., Villegas, A.M., \& Freedson-Gonzalez, M. (2008). Linguistically responsive teacher education: Preparing classroom teachers to teach English language learners. Journal of Teacher Education, 59(4), 361-373.

Lucero, A. (2015). Dual language teachers' use of conventional, environmental, and personal resources to support academic language development. Bilingual Research Journal, 38(1), $107-123$.

Ludhra, G., \& Jones, D. (2008). Conveying the "right" kind of message: Planning for the first language and culture within the primary classroom. English Teaching: Practice and Critique, 7(2), 56-70.

Markos, A. M. (2012). Mandated to learn, guided to reflect: Pre-service teachers' evolving understanding of English language learners. Issues in Teacher Education, 21(1), 39-57.

Mason-Williams, L. (2015). Unequal opportunities: A profile of the distribution of special education teachers. Exceptional Children, 81(2), 247-262. 
Muijs, D. (2011). Doing quantitative research in education with SPSS (2 ${ }^{\text {nd }}$ ed.). Thousand Oaks, CA: SAGE.

National Center for Education Statistics. (2016). English language learners in public schools. Washington, DC: U.S. Department of Education. Retrieved from http://nces.ed.gov/programs/coe/indicator_cgf.asp

National Collaborative on Diversity in the Teaching Force. (2004). Assessment of diversity in America's teaching force: A call to action. Washington, DC: Author. Retrieved from http://www.ate1.org/pubs/uploads/diversityreport.pdf

Nguyen, H. T., Benken, B. M., HakimButt, K., \& Zwiep, S. G. (2013). Teaching and learning in higher education: Enhancing faculty's preparation of prospective secondary teacher candidates for instructing English language learners. International Journal of Teaching and Learning in Higher Education, 25(3), 305-315.

Nieto, S. (2013). Language, literacy, and culture: Aha! Moments in personal and sociopolitical understanding. Journal of Language and Literacy Education, 9(1), 8-20.

Norman, G. (2010). Likert scales, levels of measurement and the "laws" of statistics. Advances in Health Sciences Education: Theory and Practice, 15(5), 625-632.

O'Brien, J. (2011). The system is broken and it's failing these kids: High school social studies teachers' attitudes toward training for ELLs. The Journal of Social Studies Research, 35(1), 22-38.

Palmer, D., \& Anissa Wicktor, L. (2008). A bilingual education for a monolingual test? The pressure to prepare for TAKS and its influence on choices for language of instruction in Texas elementary bilingual classrooms. Language Policy, 7(3), 217-235. 
Penuel, W. R., Fishman, B. J., Yamaguchi, R., Gallagher, L. P. (2007). What makes professional development effective? Strategies that foster curriculum implementation. American Educational Research Journal, 44(4), 921-958.

Pettigrew, T. F., Tropp, L. R., Wagner, U., \& Christ, O. (2011). Recent advances in intergroup contact theory. International Journal of Intercultural Relations. 35, 271-280.

Pettit, S. K. (2011). Teachers' beliefs about English language learners in the mainstream classroom: A review of the literature. International Multilingual Research Journal, 5(2), $123-147$.

Portner, H. (2008). Mentoring new teachers ( $3^{\text {rd }}$ ed.). Thousand Oaks, CA: SAGE.

Rhodes, C. M. (2017). A validation study of the culturally responsive teaching survey. Universal Journal of Educational Research, 5(1), 45-53.

Ritchie, J., \& Lewis, J. (Eds.). Qualitative research practice: A guide for social science students and researchers. Thousand Oaks, CA: SAGE.

Reyes, S. A., \& Kleyn, T. (2010). Teaching in two languages: A guide for K-12 bilingual educators. Thousand Oaks, CA: Corwin.

Rosenthal, R. \& Jacobson, L. (1968). Pygmalion in the classroom: Teacher expectations and pupils' intellectual development. New York, NY: Holt, Rinehart and Winston.

Salerno, A. S., \& Kibler, A. K. (2013). Before they teach: How pre-service teachers plan for linguistically diverse students. Teacher Education Quarterly, 40(4), 5-26.

Shirrell, M. \& Reininger, M. (2017). School working conditions and changes in student teachers' planned persistence in teaching. Teacher Education Quarterly, 44(2), 49-78.

Simpson Baird, A. (2015). Beyond the greatest hits: A counterstory of English learner parent involvement. School Community Journal, 25(2), 153 - 175. 
Skiba, R. J., Simmons, A. B., Ritter, S., Gibb, A. C., Rausch, M. K., Cuadrado, J., \& Chung, C. (2008). Achieving equity in special education. Exceptional Children, 74(3), 264-288.

Smith, J. (2001). Modeling the social construction of knowledge in ELT teacher education. ELT Journal, 55(3), 221-227.

Stake, R. E. (1995). The art of case study research. Thousand Oaks, CA: SAGE.

Souto-Manning, M. (2011). Playing with power and privilege: Theatre games in teacher education. Teaching and Teacher Education, 27(6), 997-1007.

Sorhagen, N. S. (2013). Early teacher expectations disproportionately affect poor children's high school performance. Journal of Educational Psychology, 105(2), 465-477.

Stanoscheck Youngs, C., \& Youngs, Jr., G. A. (2001). Predictors of mainstream teachers' attitudes toward ESL students. TESOL Quarterly, 35(1), 97-120.

Torrez, J. E. (2014). “Teachers should be like us!” Bridging migrant communities to rural Michigan classrooms. Multicultural Education, 21(3-4), 39-44.

Tran, Y. (2015). ESL pedagogy and certification: Teacher perceptions and efficacy. Journal of Education and Learning, 4(2), 28-42.

U.S. Census Bureau. (2018, August 26). American fact finder, Retrieved from http://factfinder.census.gov

Valencia, R. R. (1997). Introduction. In R. R. Valencia (Ed.), The Evolution of deficit thinking: Education thought and practice (pp. ix-xvii). London, England: RouteledgeFalmer.

Valencia, R.R. (2010). Dismantling contemporary deficit thinking: Educational thought and practice. New York, NY: Routeledge.

VanPatten, B., \& Williams, J. (2014). Theories in second language acquisition. New York, NY: Routeledge. 
Vinz, R. (1997). Capturing a moving form: 'Becoming' as teachers. English Education, 29(2), $137-146$.

Villegas, A. M. \& Lucas, T. (2007). The culturally responsive teacher. Educational Leadership, 64(6), 28-33.

Wang, Y. (2009). Language, parents' involvement, and social justice: The fight for maintaining minority home language: A Chinese-language case study. Multicultural Education, 16(4), 13-18.

Ward, J. (2013, September 24). Special report-New immigrants: Breaking down the barrier. The Register-Mail. Retrieved from http://www.galesburg.com/article/20130924/NEWS/130929899

Wessels, S. (2014). Supporting English and Spanish literacy through a family literacy program. School Community Journal, 24(2), 147-164.

Winstead, L. \& Wang, C. (2017). From ELLs to bilingual teachers: Spanish-English speaking Latino teachers' experiences of language shame and loss. Multicultural Education, 24 (3 - 4), 16-25.

Wlodkowski, R.J. \& Ginsberg, M. B. (1995). A framework for culturally responsive teaching. Strengthening Student Engagement, 53(1), 17-21.

Wlodkowski, R.J. \& Ginsberg, M. B. (2009). Professional learning to promote motivation and academic performance among diverse adults. In D. Bamford-Reese, B. Klein-Collins, and J. Wertheim (eds.) CAEL Forum and News: Learning Never Ends, Special Issue, 23-32.

Wong, V.W., Ruble, L. A., Yu, Y., \& McGrew, J. H. (2017). Too stressed to teach? Teaching quality, student engagement, and IEP outcomes. Exceptional Children, 83(4), 412-427. Yin, R. K. (2014). Case study research: Design and methods. Thousand Oaks, CA: SAGE. 
Yoon, B. (2012). Junsuk and Junhyuck: Adolescent immigrants' educational journey to success and identity negotiation. American Educational Research Journal, 49(5), 971-1002.

Zahner, W. (2015). The rise and run of a computational understanding of slope in a conceptually focused bilingual algebra class. Educational Studies in Mathematics, 88(1), 19-41.

Zigterman, B. (2016a, September 27). District 205 administration proposes levy increases. The Register-Mail. Retrieved from http://www.galesburg.com/news/20160927/district-205administration-proposes-levy-increases

Zigterman, B. (2016b, Jan 12). District 205 budget cuts an 'agonizing' process. The RegisterMail. Retrieved from http://www.galesburg.com/news/20160112/district-205-budgetcuts-agonizing-process 


\section{APPENDIX A: JGT AND LRT STRATEGY LIST}

\begin{tabular}{|c|}
\hline JGT Strategies \\
\hline Teacher uses predictable and consistent classroom management routines (JGT) \\
\hline Class work is at an appropriate challenge level. (JGT) \\
\hline Students are engaged and participating (JGT) \\
\hline Students and teachers appear connected and respect one another. (JGT) \\
\hline Students have a positive disposition toward learning (JGT) \\
\hline Lesson is made personal and relevant to learner. (JGT). \\
\hline Teacher provides opportunities for student choice. (JGT). \\
\hline Lesson plan or discussion includes clear goals and learning objectives (JGT) \\
\hline Students are provided with time to practice, apply or transfer new learning (JGT) \\
\hline Teachers provide feedback on both correct and incorrect responses (JGT) \\
\hline Teachers offer students time to review and practice during the lesson (JGT) \\
\hline $\begin{array}{l}\text { Teachers use multiple forms of assessment to gauge progress and offer re-teaching } \\
\text { opportunities as needed. (JGT) }\end{array}$ \\
\hline Evidence that teachers use mastery learning (JGT) \\
\hline Student perspectives and values are respected in the classroom. (JGT). \\
\hline Student is exposed to rich content with higher order thinking skills (JGT) \\
\hline Lesson has a clear structure and design (JGT) \\
\hline Pace of lesson is appropriate (JGT) \\
\hline Evidence of teacher use of portfolio assessment (JGT) or multiple forms of assessment. \\
\hline Evidence that the teacher replies to student contributions in non-evaluative ways (JGT) \\
\hline Students are provided with choice in their learning activities (JGT) \\
\hline Teachers use direct and concrete experience to teach (JGT) \\
\hline Teachers draw upon student experiences and past learning in their teaching (JGT) \\
\hline Teachers use student models, sentence frames, or other examples for student imitation (JGT) \\
\hline Students are given opportunities to work together (JGT) \\
\hline Teachers make use of peer tutors. (JGT) \\
\hline Teachers use or facilitate student-led discussions (JGT) \\
\hline Students have choice in classroom themes (JGT) \\
\hline Teachers take time to get to know students individually. (JGT) \\
\hline Teachers work to create a supportive and risk-free environment. (JGT) \\
\hline $\begin{array}{l}\text { Teachers recognize that a person's worldview is mediated by factors including race, ethnicity, } \\
\text { gender, and social class. (JGT) }\end{array}$ \\
\hline Teachers hold affirming views of diversity. (JGT) \\
\hline Teachers call upon EBP students. (JGT) \\
\hline Teachers use sufficient wait time for students to respond. (JGT) \\
\hline Teachers probe for more information and clarity from student responses. (JGT) \\
\hline Teachers establish and enforce rules that respect all students. (JGT) \\
\hline Teachers minimize competition. (JGT) \\
\hline Teachers encourage cooperation. (JGT) \\
\hline $\begin{array}{l}\text { Vocabulary is taught explicitly by embedding it into meaningful contexts and providing } \\
\text { students an opportunity to repeat the word appropriately, see the word, and encountering it in } \\
\text { various contexts (as opposed to only looking up the word in the dictionary and using it in a }\end{array}$ \\
\hline
\end{tabular}


sentence). (JGT)

Teachers highlight important information and vocab (JGT)

Students are given study guides that define vocabulary and key concepts (JGT)

Teachers use a combination of interactive and direct teaching. (JGT).

The process approach to reading is used. (JGT).

Evidence of teacher teaching or providing phonemic awareness strategies (JGT)

Evidence of teacher teaching phonics (JGT)

Evidence of teacher teaching comprehension strategies (JGT)

Teachers discuss text to promote comprehension (JGT)

Teachers and/or students summarize and paraphrase text (JGT)

Teachers provide ample reading practice (JGT)

Teacher provides ample writing opportunities (JGT)

Teacher models word-choosing strategies in writing practice. (JGT)

Teacher offers ample reading opportunities to model writing. (JGT)

Teacher explicitly teaches rhetorical conventions. (JGT)

Teacher explicitly teaches the difference between quoting, paraphrasing, and plagiarizing.

(JGT)

Teacher offers ample opportunity for extended interactions with peers and teachers. (JGT).

Teacher asks how and why questions to which they don't already know the answer. (JGT)

Teacher uses facilitative instructional conversations. (JGT)

Teachers provide outlines for lessons to students. (JGT).

Teachers make use of peer translators. (LRT).

Strategic seating (JGT)

Reads test and directions orally. (JGT).

\section{LRT Strategies}

Class materials and themes are culturally appropriate (LRT)

Students are given an opportunity to compare their culture with American culture (LRT)

Routines, rules, schedules, and expectations are presented visually in the classroom and teacher refers to these with students. (LRT)

Teacher includes specific language goals as well as content goals, which may include the specific language demands of the content area (LRT)

Evidence that teacher is familiar with student proficiency levels (LRT)

Lesson content teaches language features of content area. (LRT).

Teacher accepts non-verbal responses to oral questions. (LRT)

Students have access to and are encouraged to use bilingual dictionaries (LRT)

Teachers provide opportunities to compare American culture with students' home culture (LRT)

Teachers provide strategies for students to build background knowledge in their first language (LRT)

Teachers use visual cues, graphic organizers, hands-on activities, pictures, illustrations, maps, videos, graphic organizers, graphs, timelines, Venn diagrams, realia, and other extra-linguistic supports. (LRT)

Teachers use movements and physical gestures to communicate information. (LRT).

Teachers repeat key ideas and build redundancy into teaching. (LRT)

Teachers provide multiple ways of giving directions (orally, writing it down, etc.) (LRT) 


\begin{tabular}{|l|}
\hline $\begin{array}{l}\text { Teachers talk about time spent outside of class learning about culture and language of students } \\
\text { (LRT) }\end{array}$ \\
\hline $\begin{array}{l}\text { Teachers are careful to questions stereotypical beliefs about student's families and culture. } \\
\text { (LRT) }\end{array}$ \\
\hline $\begin{array}{l}\text { Teachers have an understanding of BICS and CALP and work to explicitly teach toward the } \\
\text { child's language needs (LRT) }\end{array}$ \\
\hline The process approach to writing is used (LRT). \\
\hline $\begin{array}{l}\text { Teaches work individually or in small groups with EBP students to teach the English sound } \\
\text { system, vocabulary, English grammar and/or discourse structure. (LRT). }\end{array}$ \\
\hline $\begin{array}{l}\text { Teachers have a solid understanding of their students' native language abilities, especially in } \\
\text { reading and writing and their previous academic preparation. (LRT) }\end{array}$ \\
\hline $\begin{array}{l}\text { Teachers supplement, modify, adapt, and/or re-write written text for EBP students to aid in } \\
\text { comprehension. (LRT) }\end{array}$ \\
\hline Teachers offer resources for teaching reading in the child's first language (LRT) \\
\hline $\begin{array}{l}\text { Teacher explicitly teaches similarities between the home language and English, such as } \\
\text { cognates, spelling and comprehension similarities. (LRT). }\end{array}$ \\
\hline $\begin{array}{l}\text { Teacher ensures that student knows the meaning of a word in English before asking the child } \\
\text { to sound it out (LRT). }\end{array}$ \\
\hline $\begin{array}{l}\text { Teaches ensure that students are able to perceive and produce distinctive sounds in English } \\
\text { before isolating those sounds in reading (LRT). }\end{array}$ \\
\hline Teacher encourages child to read in his or her home language and English (LRT) \\
\hline Teacher explicitly teaches American sentence structures (LRT) \\
\hline Teacher explicitly teaches organization of a written piece. (LRT). \\
\hline Teacher encourages and allows for a first draft in the child's home language. (LRT). \\
\hline $\begin{array}{l}\text { With newcomers, teachers speak slowly, deliberately with clear vocabulary and diction, and by } \\
\text { pausing more frequently for longer periods of time. (LRT) }\end{array}$ \\
\hline Teachers avoid idiomatic language or uses it after having defined it. (LRT) \\
\hline Teachers encourage students to speak their native languages with families and friends. (LRT) \\
\hline Teachers offer opportunities for students to write for meaningful purposes. (LRT). \\
\hline Share child's culture with class. (LRT) \\
\hline \hline
\end{tabular}




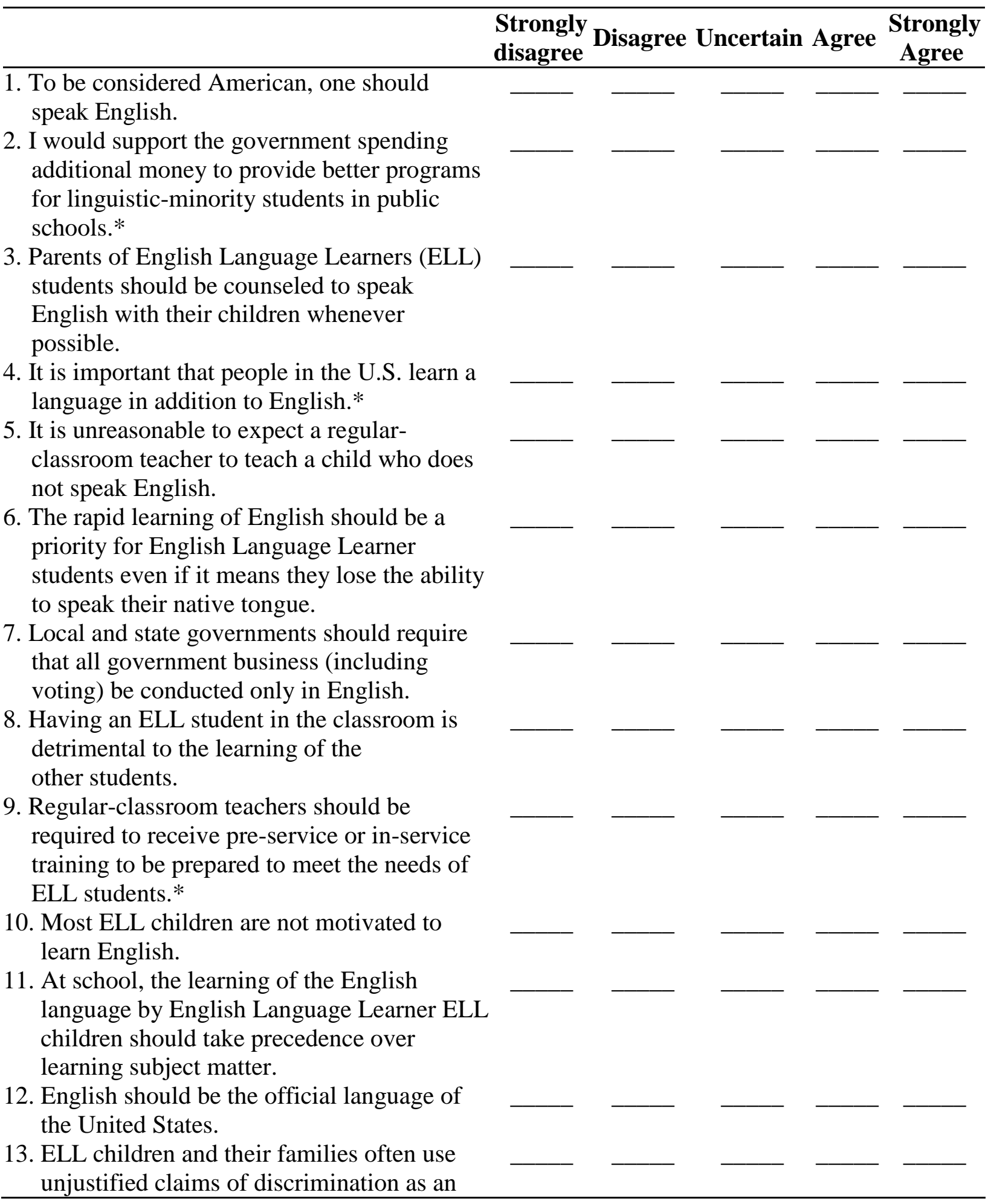


excuse for not doing well in school.

* Indicates reverse coding. All items were scored on a Likert scale.

\section{Resource Information}

14) Have you worked with an ELL student in the past?

_ Yes, frequently ___ Yes, occasionally ___ Rarely __ Never

15) If "yes" to question number 14, how often do you use the following resources to help you work with your ELL students:

\section{Never Rarely Monthly Weekly Daily}

Building ELL teacher

Books or websites for teaching ELL

students

Teaching Experience

Personal experience learning a foreign

language

Knowledge of how language is learned

District online help kit

Other teachers within the district

Administrator

Professional Development

Other (define):

Other (define): 
Other (define):

\section{Teaching Strategies:}

Please indicate how often you use the following teaching strategies.

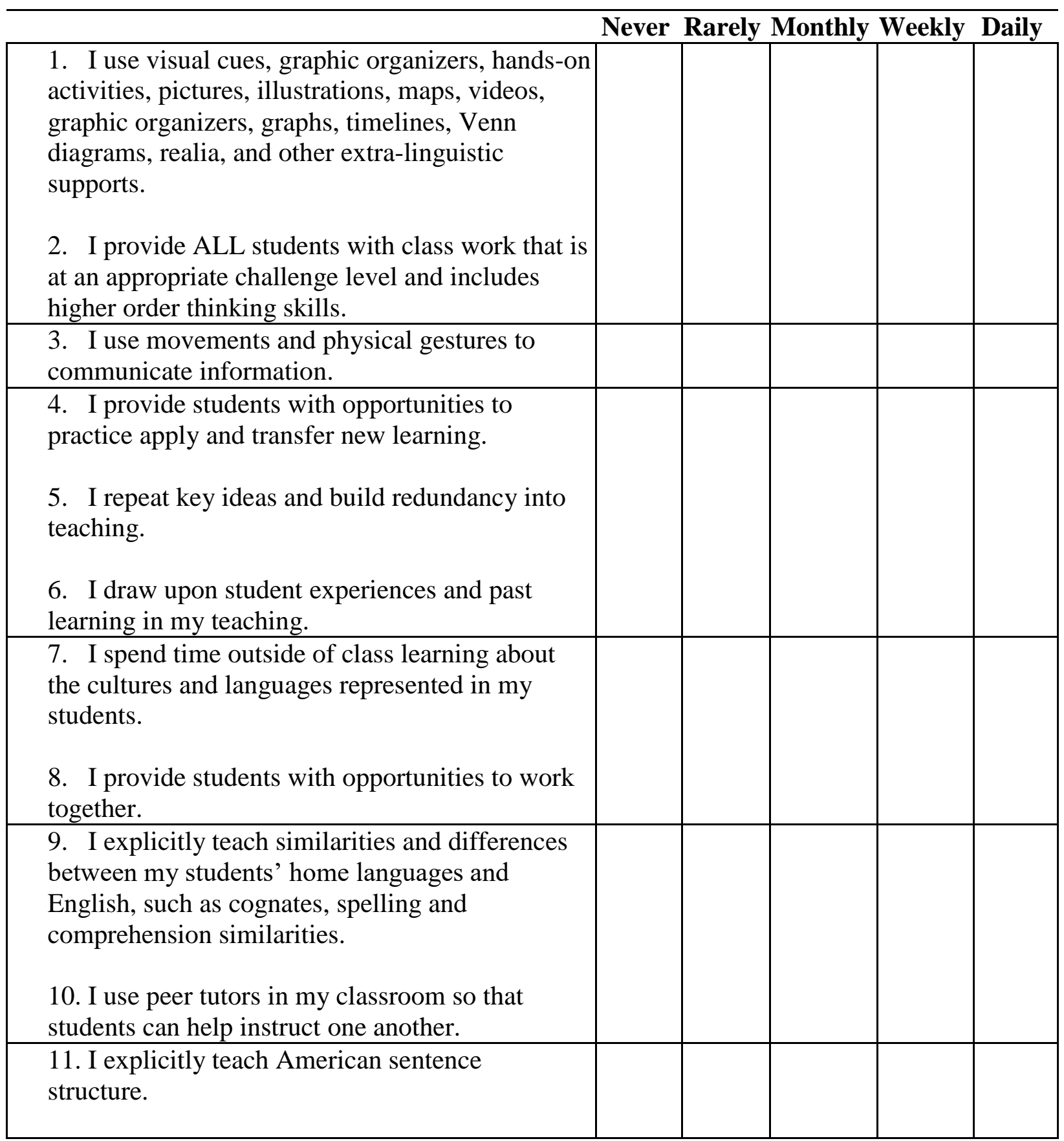




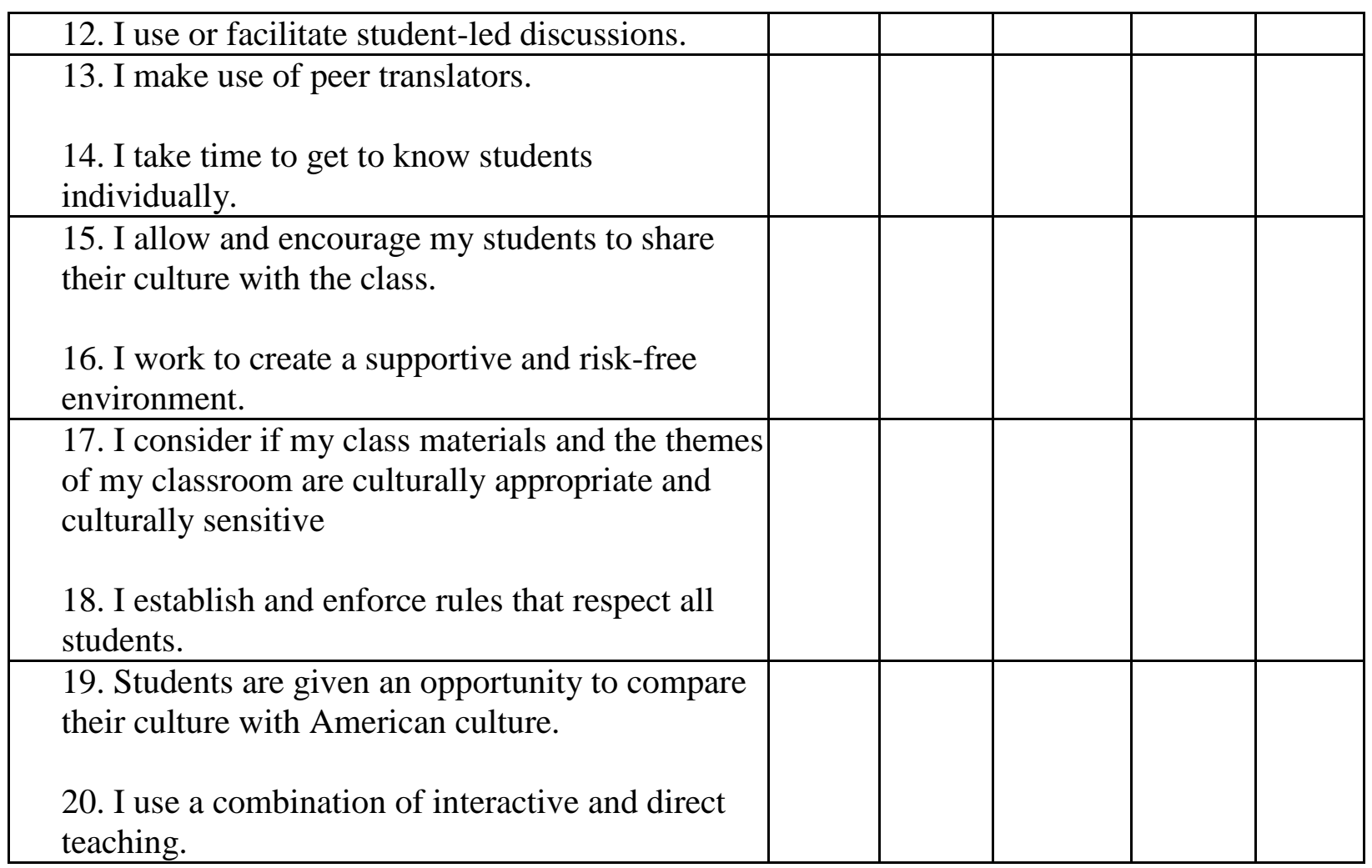

\section{Demographic Information:}

16) What is your role in the school? Classroom teacher School Specialist Specials Teacher Other

17) Do you speak a language other than English? No Somewhat Fluently

18) Have you ever been in a country where you did not understand the language? Yes No

19) How much time have you spent in a country where you did not understand the language? $<1$ month 2 - 5 months 6 - 12 months $>1$ year

20) How many years have you taught?

$1-5$ 6-10 $11-15$ $16-20$ $20+$ years 
21) How many years have you taught in this school district?

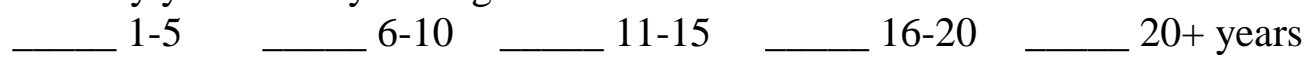

22) What is your age?

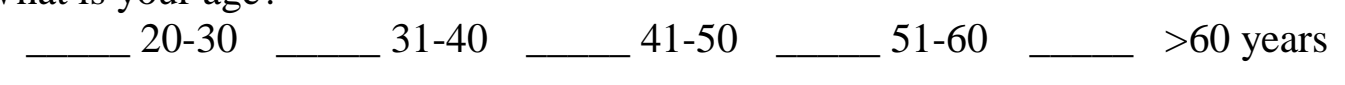

23) What grade level do you teach? $12^{\text {th }}$ grade

Pre-K-2 $2^{\text {nd }}$ grade $\_3^{\text {rd }}-5^{\text {th }}$ grade $\quad-6^{\text {th }}-8^{\text {th }}$ grade $\quad-9^{\text {th }}-$

24) What training have you had on working with ELL students?

No Training
Conference workshops

25) Before this school year, how many ELL students have you worked with in your career?

$\longleftarrow<5-6-10 \_11-15 \_16-20 \quad 20+$




\section{APPENDIX C: SHELTERED INSTRUCTION OBSERVATION PROTOCOL}

The Sheltered Instruction Observation Protocol (SIOP) (Echevarria, Vogt, \& Short, 2000;

2004; 2008)

Observer:

Teacher:

Date:

School:

Grade:

Class/Topic:

ESL Level:

Lesson: (check one) __ Multiday ___ Single-day

Directions: Check the box that best reflects what you observe in a sheltered lesson. You may give a score from 0-4 (or NA on selected items). Cite under Comments specific examples of the behaviors observed.

\begin{tabular}{|c|c|c|c|c|c|c|}
\hline & $\begin{array}{c}\text { High } \\
\text { ly } \\
\text { Evid } \\
\text { ent }\end{array}$ & & $\begin{array}{l}\text { Some } \\
\text { what } \\
\text { Eviden } \\
\mathrm{t}\end{array}$ & & $\begin{array}{c}\text { Not } \\
\text { Evid } \\
\text { ent }\end{array}$ & N/A \\
\hline Lesson Preparation & 4 & 3 & 2 & 1 & 0 & \\
\hline $\begin{array}{l}\text { 1. Content objectives clearly defined, displayed, } \\
\text { and reviewed with students }\end{array}$ & $\mathrm{O}$ & $\mathrm{O}$ & $\mathrm{O}$ & $\mathrm{O}$ & $\mathrm{O}$ & $\mathrm{O}$ \\
\hline $\begin{array}{l}\text { 2. Language objectives clearly defined, display } \\
\text { and reviewed with students }\end{array}$ & $\mathrm{O}$ & $\bigcirc$ & $\bigcirc$ & $\mathrm{O}$ & $\mathrm{O}$ & \\
\hline $\begin{array}{l}\text { 3. Content concepts appropriate for age and } \\
\text { educational background level of students }\end{array}$ & $\mathrm{O}$ & $\mathrm{O}$ & $\bigcirc$ & $\mathrm{O}$ & $\mathrm{O}$ & \\
\hline $\begin{array}{l}\text { 4. Supplementary materials used to a high } \\
\text { degree, making the lesson clear and meaningful } \\
\text { (e.g., computer programs, graphs, models, } \\
\text { visuals) }\end{array}$ & $\mathrm{O}$ & $\mathrm{O}$ & $\mathrm{O}$ & $\mathrm{O}$ & $\mathrm{O}$ & \\
\hline $\begin{array}{l}\text { 5. Adaptation of content (e.g., text, assignment) } \\
\text { to all levels of student proficiency }\end{array}$ & $\mathrm{O}$ & $\mathrm{O}$ & $\bigcirc$ & $\mathrm{O}$ & $\mathrm{O}$ & $\bigcirc$ \\
\hline $\begin{array}{l}\text { 6. Meaningful activities that integrate lesson } \\
\text { concepts (e.g., surveys, letter writing, simulations, } \\
\text { constructing models) with language practice } \\
\text { opportunities for reading, writing, listening, } \\
\text { and/or speaking }\end{array}$ & $\mathrm{O}$ & $\mathrm{O}$ & $\mathrm{O}$ & $\mathrm{O}$ & $\mathrm{O}$ & \\
\hline \multicolumn{7}{|l|}{ Comments: } \\
\hline Building Background & 4 & 3 & 2 & 1 & 0 & N/A \\
\hline $\begin{array}{l}\text { 7. Concepts explicitly linked to students' } \\
\text { background experiences }\end{array}$ & $\mathrm{O}$ & $\mathrm{O}$ & $\mathrm{O}$ & $\mathrm{O}$ & $\mathrm{O}$ & $\mathrm{O}$ \\
\hline 8. Links explicitly made between past learning & 0 & 0 & 0 & $\mathrm{O}$ & 0 & \\
\hline
\end{tabular}




\begin{tabular}{|c|c|c|c|c|c|c|}
\hline and new concepts & & & & & & \\
\hline $\begin{array}{l}\text { 9. Key vocabulary emphasized (e.g., introduced, } \\
\text { written, repeated, and highlighted for students to } \\
\text { see) }\end{array}$ & $\mathrm{O}$ & $\mathrm{O}$ & $\mathrm{O}$ & $\mathrm{O}$ & $\mathrm{O}$ & \\
\hline Comments: & & & & & & \\
\hline Comprehensible Input & 4 & 3 & 2 & 1 & 0 & \\
\hline $\begin{array}{l}\text { 10. Speech appropriate for students' proficiency } \\
\text { level (e.g., slower rate, enunciation, and simple } \\
\text { sentence structure for beginners) }\end{array}$ & O & $\mathrm{O}$ & $\mathrm{O}$ & $\mathrm{O}$ & $\mathrm{O}$ & \\
\hline 11. Clear explanation of academic tasks & $\mathrm{O}$ & $\mathrm{O}$ & $\mathrm{O}$ & $\mathrm{O}$ & $\mathrm{O}$ & \\
\hline $\begin{array}{l}\text { 12. A variety of techniques used to make content } \\
\text { concepts clear (e.g., modeling, visuals, hands-on } \\
\text { activities, demonstrations, gestures, body } \\
\text { language) }\end{array}$ & O & O & $\mathrm{O}$ & $\mathrm{O}$ & $\mathrm{O}$ & \\
\hline Comments: & & & & & & \\
\hline Strategies & 4 & 3 & 2 & 1 & 0 & $\mathrm{~N} / \mathrm{A}$ \\
\hline $\begin{array}{l}\text { 13. Ample opportunities provided for students to } \\
\text { use learning strategies }\end{array}$ & $\mathrm{O}$ & $\mathrm{O}$ & $\mathrm{O}$ & $\mathrm{O}$ & $\mathrm{O}$ & \\
\hline $\begin{array}{l}\text { 14. Scaffolding techniques consistently used } \\
\text { assisting and supporting student understanding } \\
\text { (e.g., think-alouds) }\end{array}$ & $\mathrm{O}$ & $\mathrm{O}$ & $\mathrm{O}$ & $\mathrm{O}$ & $\mathrm{O}$ & \\
\hline $\begin{array}{l}\text { 15. A variety of questions or tasks that promote } \\
\text { higher-order thinking skills (e.g., literal, } \\
\text { analytical, and interpretive questions) }\end{array}$ & $\mathrm{O}$ & $\mathrm{O}$ & $\mathrm{O}$ & $\mathrm{O}$ & $\mathrm{O}$ & \\
\hline Comments: & & & & & & \\
\hline Interaction & 4 & 3 & 2 & 1 & 0 & $\mathrm{~N} / \mathrm{A}$ \\
\hline $\begin{array}{l}\text { 16. Frequent opportunities for interaction and } \\
\text { discussion between teacher/student and among } \\
\text { students, which encourage elaborated responses } \\
\text { about lesson concepts }\end{array}$ & $\mathrm{O}$ & $\mathrm{O}$ & $\mathrm{O}$ & $\mathrm{O}$ & $\mathrm{O}$ & \\
\hline $\begin{array}{l}\text { 17. Grouping configurations support language } \\
\text { and content objectives of the lesson }\end{array}$ & $\mathrm{O}$ & $\mathrm{O}$ & $\mathrm{O}$ & $\mathrm{O}$ & $\mathrm{O}$ & \\
\hline $\begin{array}{l}\text { 18. Sufficient wait time for student responses } \\
\text { consistently provided }\end{array}$ & $\mathrm{O}$ & $\mathrm{O}$ & $\mathrm{O}$ & $\mathrm{O}$ & $\mathrm{O}$ & \\
\hline $\begin{array}{l}\text { 19. Ample opportunities for students to clarify } \\
\text { key concepts in L1 as needed with aide, peer, or } \\
\text { L1 text }\end{array}$ & $\mathrm{O}$ & $\mathrm{O}$ & $\mathrm{O}$ & $\mathrm{O}$ & $\mathrm{O}$ & $\mathrm{O}$ \\
\hline Comments & & & & & & \\
\hline Practice and Application & 4 & 3 & 2 & 1 & 0 & $\mathrm{~N} / \mathrm{A}$ \\
\hline
\end{tabular}




\begin{tabular}{|c|c|c|c|c|c|c|}
\hline $\begin{array}{l}\text { 20. Hands-on materials and/or manipulatives } \\
\text { provided for students to practice using new } \\
\text { content } \\
\text { knowledge }\end{array}$ & O & O & 0 & O & O & O \\
\hline content & O & O & 0 & O & O & $\bigcirc$ \\
\hline 22. Activities integrate all language skills (i.e., & $\mathrm{O}$ & $\bigcirc$ & $\mathrm{O}$ & $\bigcirc$ & $\mathrm{O}$ & \\
\hline Comments: & & & & & & \\
\hline Lesson Delivery & 4 & 3 & 2 & 1 & 0 & N/A \\
\hline $\begin{array}{l}\text { 23. Content objectives clearly supported by } \\
\text { lesson } \bigcirc \stackrel{\bigcirc}{\text { delivery }}\end{array}$ & $\bigcirc$ & $\mathrm{O}$ & O & $\mathrm{O}$ & $\bigcirc$ & \\
\hline $\begin{array}{l}\text { 24. Language objectives clearly supported by } \\
\text { lesson } \bigcirc \\
\text { delivery }\end{array}$ & $\mathrm{O}$ & $\mathrm{O}$ & $\mathrm{O}$ & $\mathrm{O}$ & $\mathrm{O}$ & \\
\hline $\begin{array}{l}\text { 25. Students engaged approximately } 90 \% \text { to } \\
100 \% \text { of } \bigcirc \\
\text { the period }\end{array}$ & $\mathrm{O}$ & $\mathrm{O}$ & $\mathrm{O}$ & $\mathrm{O}$ & $\mathrm{O}$ & \\
\hline $\begin{array}{l}\text { 26. Pacing of the lesson appropriate to students' } \\
\text { ability } \bigcirc \\
\text { level }\end{array}$ & $\mathrm{O}$ & O & $\mathrm{O}$ & $\mathrm{O}$ & $\mathrm{O}$ & \\
\hline Comments: & & & & & & \\
\hline Review and Assessment & 4 & 3 & 2 & 1 & 0 & N/A \\
\hline 27. Comprehensive review of key vocabulary & $\mathrm{O}$ & $\mathrm{O}$ & $\mathrm{O}$ & $\mathrm{O}$ & $\mathrm{O}$ & \\
\hline $\begin{array}{l}\text { 28. Comprehensive review of key content } \\
\text { concepts }\end{array}$ & $\mathrm{O}$ & $\mathrm{O}$ & $\mathrm{O}$ & $\mathrm{O}$ & $\mathrm{O}$ & \\
\hline $\begin{array}{l}\text { 29. Regular feedback provided to students on } \\
\text { their output (e.g., language, content, work) }\end{array}$ & $\mathrm{O}$ & $\mathrm{O}$ & $\mathrm{O}$ & $\mathrm{O}$ & $\mathrm{O}$ & \\
\hline
\end{tabular}




\begin{tabular}{|l|c|c|c|c|c|c|}
\hline $\begin{array}{l}\text { 30. Assessment of student comprehension } \\
\text { and } \\
\text { learning of all lesson objectives (e.g., spot } \\
\text { checking, } \\
\text { group response) throughout the lesson }\end{array}$ & $\bigcirc$ & $\bigcirc$ & $\bigcirc$ & $\bigcirc$ & $\bigcirc$ & \\
\hline Comments: & & & & & & \\
\hline Classroom Environment & 4 & 3 & 2 & 1 & 0 & N/A \\
\hline 31. Evidence of including home languages. & $\bigcirc$ & $\bigcirc$ & $\bigcirc$ & $\bigcirc$ & $\bigcirc$ & \\
\hline 32. Evidence of including home cultures. & $\bigcirc$ & $\bigcirc$ & $\bigcirc$ & $\bigcirc$ & $\bigcirc$ & \\
\hline 33. Evidence of family involvement. & $\bigcirc$ & $\bigcirc$ & $\bigcirc$ & $\bigcirc$ & $\bigcirc$ & \\
\hline Comments: & & & & & & \\
\hline
\end{tabular}




\section{APPENDIX D: DOCUMENT ANALYSIS PROTOCOL}

Document Analysis

\begin{tabular}{|c|c|c|c|}
\hline \multicolumn{4}{|l|}{ Participant } \\
\hline \multicolumn{4}{|l|}{ Topic/Unit } \\
\hline Type of Document & Lesson Plan & Handout & Assignment \\
\hline & Assessment & Other (D & \\
\hline
\end{tabular}

\begin{tabular}{|l|l|l|}
\hline Do lesson plans demonstrate any of the following... & Yes/No & Qnty \\
\hline 1) Specific language objectives? & & \\
\hline 2) Considerations for the culture of students? & & \\
\hline 3) Connections with literature or journaling? & & \\
\hline 4) Relevant knowledge of student home language? & & \\
\hline 5) Techniques used to scaffold language/model English? & & \\
\hline 6) Specific vocabulary? & & \\
\hline 7) Visual explanations? & & \\
\hline 8) Use of Reala? & & \\
\hline 9) Written materials in the students' home languages? & & \\
\hline 10) Opportunity for students to write in their home language? & & \\
\hline 11) If relevant, alternative methods of completing mathematical procedures are \\
accepted, encouraged, and used for scaffolding instruction. & & \\
\hline 12) Other: & & \\
\hline Notes: & & \\
\hline
\end{tabular}




\begin{tabular}{|l|l|l|}
\hline Assignment or Assessment & Yes/No & Qnty \\
\hline Have appropriate modifications and/or accommodations been made? & & \\
\hline $\begin{array}{l}\text { Are accommodations and/or modifications tailored to the needs of individual } \\
\text { emergent bilingual students? }\end{array}$ & & \\
Notes: & & \\
\hline $\begin{array}{l}\text { Is there evidence that accommodations and/or modifications match the } \\
\text { accommodations and/or modifications that were present at the time of } \\
\text { instruction? } \\
\text { Notes: }\end{array}$ & & \\
\hline
\end{tabular}




\section{APPENDIX E: INITIAL INTERVIEW QUESTIONS}

\section{First Interview}

Thank you for agreeing to meet with me. Your participation in this research will help the school district identify:

- the resources you find most useful in working with your ESL students,

- the effective teaching strategies you are currently using with your ESL students, and

- what has helped to form your practices, thoughts, attitudes, and beliefs toward working with your ESL students.

I want to remind you that your participation in this research is voluntary and you have the right to withdraw from participation at any time.

\begin{tabular}{|c|c|}
\hline Research Questions & Interviewer Questions \\
\hline $\begin{array}{l}\text { 1. What influences the } \\
\text { dispositions that } \\
\text { teachers hold toward } \\
\text { their emergent bilingual } \\
\text { students? }\end{array}$ & $\begin{array}{l}\text { 1) How long have you worked at this school? What did you } \\
\text { do before you taught here? (Probe for information } \\
\text { regarding experiences of the teacher in general) } \\
\text { 2) Have you worked with an ELL student in the past? } \\
\text { Can you tell me about that experience? (probe for what } \\
\text { year this was, the language the student spoke, their level } \\
\text { of English proficiency, general thoughts/feelings about } \\
\text { how the teacher felt working with this/these student(s) } \\
\text { 3) Do you feel comfortable knowing about what strategies } \\
\text { work well with ELL students? } \\
\text { 4) Can you tell me more about that? }\end{array}$ \\
\hline $\begin{array}{l}\text { 2. What resources and } \\
\text { strategies do teachers } \\
\text { draw upon in working } \\
\text { with their emergent } \\
\text { bilingual students? }\end{array}$ & $\begin{array}{l}\text { 5) You are assigned to work with an ELL student this year. } \\
\text { How many ELL students do you have in your classroom } \\
\text { this year? } \\
\text { 6) If you feel that you need more help in working with your } \\
\text { ELL students, what resources do you think you are going } \\
\text { to turn to first? These resources could include materials in } \\
\text { the classroom, ideas found online, other teachers in the } \\
\text { building, reflecting on your own teaching experience, etc. } \\
\text { 7) How do you think this/these resources will be most useful } \\
\text { to you? } \\
\text { 8) I would like to observe your classroom } 2-3 \text { times this }\end{array}$ \\
\hline
\end{tabular}




\begin{tabular}{|l|l|}
\hline $\begin{array}{l}\text { semester(?). Can you tell me what teaching strategies I } \\
\text { will get to see in your room? }\end{array}$ \\
$\begin{array}{l}\text { 9) Can you tell me more about why these teaching strategies } \\
\text { are going to be useful in working with your students? }\end{array}$ \\
10) Is there anything else you would like me to know?
\end{tabular}

Thank you so much for your time today! I am looking forward to observing your classroom. If you have any questions or if you would like to add anything to what we talked about today, please do not hesitate to contact me. You can reach me on the district e-mail or call me on my cell phone. 


\section{APPENDIX F: FOLLOW-UP INTERVIEW QUESTIONS}

\section{Second Interview}

Thank you for agreeing to meet with me again and thank you for allowing me to visit your classroom. As a reminder, your participation in this research will help the school district identify:

- the resources you find most useful in working with your ESL students,

- the effective teaching strategies you are currently using with your ESL students, and

- what has helped to form your practices, thoughts, attitudes, and beliefs toward working with your ESL students.

Also, please remember that your participation in this research is voluntary and you have the right to withdraw from participation at any time.

\begin{tabular}{|c|c|}
\hline Researcher Questions & Interviewer Questions \\
\hline $\begin{array}{l}\text { 1. What influences the } \\
\text { dispositions that teachers } \\
\text { hold toward their } \\
\text { emergent bilingual } \\
\text { students? }\end{array}$ & $\begin{array}{l}\text { 1) How would you describe your overall reaction to } \\
\text { working with ESL students in your classroom? (If } \\
\text { necessary, ask "Would you describe this as an } \\
\text { enjoyable experience, a challenge, a bit of both? Can } \\
\text { you tell me more about this?"). }\end{array}$ \\
\hline $\begin{array}{l}\text { 2. What resources and } \\
\text { strategies do teachers } \\
\text { draw upon in working } \\
\text { with their emergent } \\
\text { bilingual students? } \\
\text { 3. How does a teacher's } \\
\text { disposition inform the } \\
\text { resources and strategies } \\
\text { they draw upon in } \\
\text { working with emergent } \\
\text { bilingual students? } \\
\text { 4. What is the interplay } \\
\text { between the teaching } \\
\text { strategies and resources } \\
\text { that teachers use in } \\
\text { working with emergent } \\
\text { bilingual students? }\end{array}$ & $\begin{array}{l}\text { 2) What resources do you find yourself turning to the most } \\
\text { when developing your lessons for your ELL students? } \\
\text { (If necessary, say, "Resources can include materials in } \\
\text { the classroom, ideas found online, other teachers in the } \\
\text { building, reflecting on your own teaching experience, or } \\
\text { other things of that nature.") } \\
\text { 3) Why do you think these are the resources you use? } \\
\text { 4) Are there any resources that you have available that you } \\
\text { do not use as often? } \\
\text { 5) Can you tell me more about this? } \\
\text { 6) What teaching strategies do you use the most in your } \\
\text { classroom? (If necessary, say, "Teaching strategies may } \\
\text { include modifications or accommodations, using } \\
\text { materials in the home language, or other teaching } \\
\text { methods that you know really help your ELL students.") } \\
\text { 7) Can you tell me more about how these teachings } \\
\text { strategies are helpful? } \\
\text { 8) Are there any teaching strategies that you have }\end{array}$ \\
\hline
\end{tabular}




\begin{tabular}{|l|l|}
\hline Researcher Questions & Interviewer Questions \\
\hline & considered using but have not tried yet? \\
9) Can you tell me more about these? \\
$\begin{array}{l}\text { 10) Is there anything else you'd like me to know about } \\
\text { working with your ELL students? }\end{array}$ \\
$\begin{array}{l}\text { 11) If you were told that you could expect two or three } \\
\text { ESL students in one of your classes next year, how } \\
\text { would you describe your reaction? } \\
\text { 12) Can you tell me more about that? } \\
\text { 13) Is there anything else you would like me to know? }\end{array}$ \\
\hline
\end{tabular}

Thank you so much for your time today and for agreeing to be in this study. If you don't mind, I'd like to email you in about a month so that I can review with you some of the main points we discussed here. If you have any questions, please do not hesitate to email me or call me. 
APPENDIX G: JGT AND LRT CHECKLIST BY TEACHER

\begin{tabular}{|c|c|c|c|c|c|c|}
\hline JGT Strategies & Holly & Linda & Melody & Jennifer & Susan & Stephanie \\
\hline $\begin{array}{l}\text { Teacher uses predictable and } \\
\text { consistent classroom } \\
\text { management routines (JGT) }\end{array}$ & & & $\mathrm{x}$ & & $\mathrm{x}$ & $\mathrm{x}$ \\
\hline $\begin{array}{l}\text { Class work is at an appropriate } \\
\text { challenge level. (JGT) }\end{array}$ & & & $\mathrm{x}$ & $\mathrm{x}$ & $\mathrm{x}$ & \\
\hline $\begin{array}{l}\text { Students are engaged and } \\
\text { participating (JGT) }\end{array}$ & & $\mathrm{x}$ & $\mathrm{x}$ & $\mathrm{x}$ & $\mathrm{x}$ & $\mathrm{x}$ \\
\hline $\begin{array}{l}\text { Students and teachers appear } \\
\text { connected and respect one } \\
\text { another. (JGT) }\end{array}$ & & $\mathrm{x}$ & & & $\mathrm{x}$ & $\mathrm{x}$ \\
\hline $\begin{array}{l}\text { Students have a positive } \\
\text { disposition toward learning } \\
\text { (JGT) }\end{array}$ & & $\mathrm{x}$ & $\mathrm{x}$ & $\mathrm{x}$ & & $\mathrm{x}$ \\
\hline $\begin{array}{l}\text { Lesson is made personal and } \\
\text { relevant to learner. (JGT). }\end{array}$ & & $\mathrm{x}$ & $\mathrm{x}$ & & & \\
\hline $\begin{array}{l}\text { Teacher provides opportunities } \\
\text { for student choice. (JGT). }\end{array}$ & $\mathrm{x}$ & & & $\mathrm{x}$ & & \\
\hline $\begin{array}{l}\text { Lesson plan or discussion } \\
\text { includes clear goals and } \\
\text { learning objectives (JGT) }\end{array}$ & & & & & & \\
\hline $\begin{array}{l}\text { Students are provided with } \\
\text { time to practice, apply or } \\
\text { transfer new learning (JGT) }\end{array}$ & $\mathrm{x}$ & $\mathrm{x}$ & $\mathrm{x}$ & $\mathrm{x}$ & $\mathrm{x}$ & $\mathrm{x}$ \\
\hline $\begin{array}{l}\text { Teachers provide feedback on } \\
\text { both correct and incorrect } \\
\text { responses (JGT) }\end{array}$ & & $\mathrm{x}$ & & $\mathrm{x}$ & $\mathrm{x}$ & $\mathrm{x}$ \\
\hline $\begin{array}{l}\text { Teachers offer students time to } \\
\text { review and practice during the } \\
\text { lesson (JGT) }\end{array}$ & & & & $\mathrm{x}$ & $\mathrm{x}$ & $\mathrm{x}$ \\
\hline $\begin{array}{l}\text { Teachers use multiple forms of } \\
\text { assessment to gauge progress } \\
\text { and offer re-teaching } \\
\text { opportunities as needed. (JGT) }\end{array}$ & $\mathrm{x}$ & & & & & $\mathrm{x}$ \\
\hline $\begin{array}{l}\text { Evidence that teachers use } \\
\text { mastery learning (JGT) }\end{array}$ & $\mathrm{x}$ & & & & & \\
\hline $\begin{array}{l}\text { Student perspectives and } \\
\text { values are respected in the } \\
\text { classroom. (JGT). }\end{array}$ & $\mathrm{x}$ & $\mathrm{x}$ & & $\mathrm{x}$ & & \\
\hline $\begin{array}{l}\text { Student is exposed to rich } \\
\text { content with higher order } \\
\text { thinking skills (JGT) }\end{array}$ & & & & $\mathrm{x}$ & $\mathrm{x}$ & $\mathrm{x}$ \\
\hline Lesson has a clear structure & & & & & $\mathrm{x}$ & $\mathrm{x}$ \\
\hline
\end{tabular}




\begin{tabular}{|c|c|c|c|c|c|c|}
\hline JGT Strategies & Holly & Linda & Melody & Jennifer & Susan & Stephanie \\
\hline \multicolumn{7}{|l|}{ and design (JGT) } \\
\hline \multicolumn{7}{|l|}{$\begin{array}{l}\text { Pace of lesson is appropriate } \\
\text { (JGT) }\end{array}$} \\
\hline $\begin{array}{l}\text { Evidence of teacher use of } \\
\text { portfolio assessment (JGT) or } \\
\text { multiple forms of assessment. }\end{array}$ & $\mathrm{x}$ & & & & & \\
\hline $\begin{array}{l}\text { Evidence that the teacher } \\
\text { replies to student contributions } \\
\text { in non-evaluative ways (JGT) }\end{array}$ & & & & & $\mathrm{x}$ & \\
\hline $\begin{array}{l}\text { Students are provided with } \\
\text { choice in their learning } \\
\text { activities (JGT) }\end{array}$ & & & & $\mathrm{x}$ & & \\
\hline $\begin{array}{l}\text { Teachers use direct and } \\
\text { concrete experience to teach } \\
\text { (JGT) }\end{array}$ & $\mathrm{x}$ & & $\mathrm{x}$ & & & $\mathrm{x}$ \\
\hline $\begin{array}{l}\text { Teachers draw upon student } \\
\text { experiences and past learning } \\
\text { in their teaching (JGT) }\end{array}$ & $\mathrm{x}$ & $\mathrm{x}$ & & $\mathrm{x}$ & $\mathrm{x}$ & \\
\hline $\begin{array}{l}\text { Teachers use student models, } \\
\text { sentence frames, or other } \\
\text { examples for student imitation } \\
\text { (JGT) }\end{array}$ & $\mathrm{x}$ & & & $\mathrm{x}$ & & \\
\hline $\begin{array}{l}\text { Students are given } \\
\text { opportunities to work together } \\
\text { (JGT) }\end{array}$ & $\mathrm{x}$ & $\mathrm{x}$ & $\mathrm{x}$ & $\mathrm{x}$ & & \\
\hline $\begin{array}{l}\text { Teachers make use of peer } \\
\text { tutors. (JGT) }\end{array}$ & & $\mathrm{x}$ & $\mathrm{x}$ & $\mathrm{x}$ & $\mathrm{x}$ & \\
\hline $\begin{array}{l}\text { Teachers use or facilitate } \\
\text { student-led discussions (JGT) }\end{array}$ & $\mathrm{x}$ & $\mathrm{x}$ & & $\mathrm{x}$ & & \\
\hline \multicolumn{7}{|l|}{$\begin{array}{l}\text { Students have choice in } \\
\text { classroom themes (JGT) }\end{array}$} \\
\hline $\begin{array}{l}\text { Teachers take time to get to } \\
\text { know students individually. } \\
\text { (JGT) }\end{array}$ & & $\mathrm{x}$ & $\mathrm{x}$ & & $\mathrm{x}$ & \\
\hline $\begin{array}{l}\text { Teachers work to create a } \\
\text { supportive and risk-free } \\
\text { environment. (JGT) }\end{array}$ & $\mathrm{x}$ & $\mathrm{x}$ & $\mathrm{x}$ & & $\mathrm{x}$ & $\mathrm{x}$ \\
\hline $\begin{array}{l}\text { Teachers recognize that a } \\
\text { person's worldview is } \\
\text { mediated by factors including } \\
\text { race, ethnicity, gender, and } \\
\text { social class. (JGT) }\end{array}$ & $\mathrm{x}$ & $\mathrm{x}$ & & & $\mathrm{x}$ & \\
\hline $\begin{array}{l}\text { Teachers hold affirming views } \\
\text { of diversity. (JGT) }\end{array}$ & $\mathrm{x}$ & $\mathrm{x}$ & & $\mathrm{x}$ & $\mathrm{x}$ & $\mathrm{x}$ \\
\hline
\end{tabular}




\begin{tabular}{|l|c|c|c|c|c|c|}
\hline JGT Strategies & Holly & Linda & Melody & Jennifer & Susan & Stephanie \\
\hline $\begin{array}{l}\text { Teachers call upon EBP } \\
\text { students. (JGT) }\end{array}$ & & $\mathrm{x}$ & $\mathrm{x}$ & $\mathrm{x}$ & $\mathrm{x}$ & $\mathrm{x}$ \\
\hline $\begin{array}{l}\text { Teachers use sufficient wait } \\
\text { time for students to respond. } \\
\text { (JGT) }\end{array}$ & & & & & $\mathrm{x}$ & \\
\hline $\begin{array}{l}\text { Teachers probe for more } \\
\text { information and clarity from } \\
\text { student responses. (JGT) }\end{array}$ & & $\mathrm{x}$ & $\mathrm{x}$ & $\mathrm{x}$ & $\mathrm{x}$ \\
\hline $\begin{array}{l}\text { Teachers establish and enforce } \\
\text { rules that respect all students. } \\
\text { (JGT) }\end{array}$ & $\mathrm{x}$ & $\mathrm{x}$ & $\mathrm{x}$ & & $\mathrm{x}$ & $\mathrm{x}$ \\
\hline $\begin{array}{l}\text { Teachers minimize } \\
\text { competition. (JGT) }\end{array}$ & $\mathrm{x}$ & $\mathrm{x}$ & $\mathrm{x}$ & $\mathrm{x}$ & & \\
\hline $\begin{array}{l}\text { Teachers encourage } \\
\text { cooperation. (JGT) }\end{array}$ & & & $\mathrm{x}$ & & $\mathrm{x}$ \\
\hline $\begin{array}{l}\text { Vocabulary is taught explicitly } \\
\text { by embedding it into } \\
\text { meaningful contexts and } \\
\text { providing students an } \\
\text { opportunity to repeat the word } \\
\text { appropriately, see the word, } \\
\text { and encountering it in various } \\
\text { contexts (as opposed to only } \\
\text { looking up the word in the } \\
\text { dictionary and using it in a } \\
\text { sentence). (JGT) }\end{array}$ & & $\mathrm{x}$ & $\mathrm{x}$ & $\mathrm{x}$ & & $\mathrm{x}$ \\
\hline $\begin{array}{l}\text { Teachers highlight important } \\
\text { information and vocab (JGT) }\end{array}$ & & & & $\mathrm{x}$ & $\mathrm{x}$ & $\mathrm{x}$ \\
\hline $\begin{array}{l}\text { Students are given study guides } \\
\text { that define vocabulary and key } \\
\text { concepts (JGT) }\end{array}$ & & & & $\mathrm{x}$ & $\mathrm{x}$ & \\
\hline $\begin{array}{l}\text { Teachers use a combination of } \\
\text { interactive and direct teaching. } \\
\text { (JGT). }\end{array}$ & $\mathrm{x}$ & & & & & \\
\hline $\begin{array}{l}\text { The process approach to } \\
\text { reading is used. (JGT). }\end{array}$ & & & & & & \\
\hline $\begin{array}{l}\text { Evidence of teacher teaching or } \\
\text { providing phonemic awareness } \\
\text { strategies (JGT) }\end{array}$ & & & & & & \\
\hline $\begin{array}{l}\text { Evidence of teacher teaching } \\
\text { phonics (JGT) }\end{array}$ & & & & & \\
\hline $\begin{array}{l}\text { Evidence of teacher teaching } \\
\text { comprehension strategies } \\
\text { (JGT) }\end{array}$ & & & & & & \\
\hline
\end{tabular}




\begin{tabular}{|c|c|c|c|c|c|c|}
\hline JGT Strategies & Holly & Linda & Melody & Jennifer & Susan & Stephanie \\
\hline $\begin{array}{l}\text { Teachers discuss text to } \\
\text { promote comprehension (JGT) }\end{array}$ & & & & $\mathrm{x}$ & $\mathrm{x}$ & \\
\hline $\begin{array}{l}\text { Teachers and/or students } \\
\text { summarize and paraphrase text } \\
\text { (JGT) }\end{array}$ & & & & & $\mathrm{x}$ & \\
\hline $\begin{array}{l}\text { Teachers provide ample } \\
\text { reading practice (JGT) }\end{array}$ & & $\mathrm{x}$ & & & & \\
\hline $\begin{array}{l}\text { Teacher provides ample } \\
\text { writing opportunities (JGT) }\end{array}$ & & & & & & \\
\hline $\begin{array}{l}\text { Teacher models word-choosing } \\
\text { strategies in writing practice. } \\
\text { (JGT) }\end{array}$ & & & & & & \\
\hline $\begin{array}{l}\text { Teacher offers ample reading } \\
\text { opportunities to model writing. } \\
\text { (JGT) }\end{array}$ & & & & & & \\
\hline $\begin{array}{l}\text { Teacher explicitly teaches } \\
\text { rhetorical conventions. (JGT) }\end{array}$ & & & & & & \\
\hline $\begin{array}{l}\text { Teacher explicitly teaches the } \\
\text { difference between quoting, } \\
\text { paraphrasing, and plagiarizing. } \\
\text { (JGT) }\end{array}$ & & & & & & \\
\hline $\begin{array}{l}\text { Teacher offers ample } \\
\text { opportunity for extended } \\
\text { interactions with peers and } \\
\text { teachers. (JGT). }\end{array}$ & $\mathrm{x}$ & $\mathrm{x}$ & & & & \\
\hline $\begin{array}{l}\text { Teacher asks how and why } \\
\text { questions to which they don't } \\
\text { already know the answer. } \\
\text { (JGT) }\end{array}$ & & & & & & \\
\hline $\begin{array}{l}\text { Teacher uses facilitative } \\
\text { instructional conversations. } \\
\text { (JGT) }\end{array}$ & $\mathrm{x}$ & & & & & \\
\hline $\begin{array}{l}\text { Teachers provide outlines for } \\
\text { lessons to students. (JGT). }\end{array}$ & & & & & & \\
\hline $\begin{array}{l}\text { Teachers make use of peer } \\
\text { translators. (LRT). }\end{array}$ & & & $\mathrm{x}$ & $\mathrm{x}$ & & \\
\hline Strategic seating (JGT) & & $\mathrm{x}$ & & & $\mathrm{x}$ & \\
\hline $\begin{array}{l}\text { Reads test and directions } \\
\text { orally. (JGT). }\end{array}$ & $\mathrm{x}$ & & & & $\mathrm{x}$ & $\mathrm{X}$ \\
\hline TOTAL JGT & 20 & 24 & 18 & 25 & 29 & 20 \\
\hline LRT Strategies & $\mathbf{H}$ & $\overline{\mathbf{L}}$ & $\overline{\mathbf{M}}$ & $\overline{\mathbf{J}}$ & Su & St \\
\hline $\begin{array}{l}\text { Class materials and themes are } \\
\text { culturally appropriate (LRT) }\end{array}$ & & & & & $\bar{x}$ & \\
\hline Students are given an & $\mathrm{x}$ & $\mathrm{x}$ & & & & \\
\hline
\end{tabular}




\begin{tabular}{|l|l|l|l|l|l|l|}
\hline JGT Strategies & Holly & Linda & Melody & Jennifer & Susan & Stephanie \\
\hline $\begin{array}{l}\text { Opportunity to compare their } \\
\text { culture with American culture } \\
\text { (LRT) }\end{array}$ & & & & & & \\
\hline $\begin{array}{l}\text { Routines, rules, schedules, and } \\
\text { expectations are presented } \\
\text { visually in the classroom and } \\
\text { teacher refers to these with } \\
\text { students. (LRT) }\end{array}$ & & & & & & \\
\hline $\begin{array}{l}\text { Teacher includes specific } \\
\text { language goals as well as } \\
\text { content goals, which may } \\
\text { include the specific language } \\
\text { demands of the content area } \\
\text { (LRT) }\end{array}$ & & & & & & \\
\hline $\begin{array}{l}\text { Evidence that teacher is } \\
\text { familiar with student } \\
\text { proficiency levels (LRT) }\end{array}$ & & & & & & \\
\hline $\begin{array}{l}\text { Lesson content teaches } \\
\text { language features of content } \\
\text { area. (LRT). }\end{array}$ & & & & & & \\
\hline $\begin{array}{l}\text { Teacher accepts non-verbal } \\
\text { responses to oral questions. } \\
\text { (LRT) }\end{array}$ & & & & & & \\
\hline $\begin{array}{l}\text { Students have access to and are } \\
\text { encouraged to use bilingual } \\
\text { dictionaries (LRT) }\end{array}$ & & & & & & \\
\hline $\begin{array}{l}\text { Teachers provide opportunities } \\
\text { to compare American culture } \\
\text { with students' home culture } \\
\text { (LRT) }\end{array}$ & & & & & & \\
\hline $\begin{array}{l}\text { Teachers provide strategies for } \\
\text { students to build background } \\
\text { knowledge in their first } \\
\text { language (LRT) }\end{array}$ & & & & & & \\
\hline $\begin{array}{l}\text { Teachers use visual cues, } \\
\text { graphic organizers, hands-on } \\
\text { activities, pictures, } \\
\text { illustrations, maps, videos, } \\
\text { graphic organizers, graphs, } \\
\text { timelines, Venn diagrams, } \\
\text { realia, and other extra- } \\
\text { linguistic supports. (LRT) }\end{array}$ & & & & & \\
\hline $\begin{array}{l}\text { Teachers use movements and } \\
\text { physical gestures to }\end{array}$ & & $\mathrm{x}$ & & & \\
\hline
\end{tabular}




\begin{tabular}{|c|c|c|c|c|c|c|}
\hline JGT Strategies & Holly & Linda & Melody & Jennifer & Susan & Stephanie \\
\hline \multicolumn{7}{|l|}{$\begin{array}{l}\text { communicate information. } \\
\text { (LRT). }\end{array}$} \\
\hline $\begin{array}{l}\text { Teachers repeat key ideas and } \\
\text { build redundancy into teaching. } \\
\text { (LRT) }\end{array}$ & & & $\mathrm{x}$ & $\mathrm{x}$ & $\mathrm{x}$ & $\mathrm{x}$ \\
\hline \multicolumn{7}{|l|}{$\begin{array}{l}\text { Teachers provide multiple } \\
\text { ways of giving directions } \\
\text { (orally, writing it down, etc.) } \\
\text { (LRT) }\end{array}$} \\
\hline $\begin{array}{l}\text { Teachers talk about time spent } \\
\text { outside of class learning about } \\
\text { culture and language of } \\
\text { students (LRT) }\end{array}$ & $\mathrm{x}$ & $\mathrm{x}$ & & & & $\mathrm{x}$ \\
\hline $\begin{array}{l}\text { Teachers are careful to } \\
\text { questions stereotypical beliefs } \\
\text { about student's families and } \\
\text { culture. (LRT) }\end{array}$ & & & & & & $\mathrm{x}$ \\
\hline \multicolumn{7}{|l|}{$\begin{array}{l}\text { Teachers have an } \\
\text { understanding of BICS and } \\
\text { CALP and work to explicitly } \\
\text { teach toward the child's } \\
\text { language needs (LRT) }\end{array}$} \\
\hline \multicolumn{7}{|l|}{$\begin{array}{l}\text { The process approach to } \\
\text { writing is used (LRT). }\end{array}$} \\
\hline \multicolumn{7}{|l|}{$\begin{array}{l}\text { Teaches work individually or } \\
\text { in small groups with EBP } \\
\text { students to teach the English } \\
\text { sound system, vocabulary, } \\
\text { English grammar and/or } \\
\text { discourse structure. (LRT). }\end{array}$} \\
\hline \multicolumn{7}{|l|}{$\begin{array}{l}\text { Teachers have a solid } \\
\text { understanding of their } \\
\text { students' native language } \\
\text { abilities, especially in reading } \\
\text { and writing and their previous } \\
\text { academic preparation. (LRT) }\end{array}$} \\
\hline \multicolumn{7}{|l|}{$\begin{array}{l}\text { Teachers supplement, modify, } \\
\text { adapt, and/or re-write written } \\
\text { text for EBP students to aid in } \\
\text { comprehension. (LRT) }\end{array}$} \\
\hline \multicolumn{7}{|l|}{$\begin{array}{l}\text { Teachers offer resources for } \\
\text { teaching reading in the child's } \\
\text { first language (LRT) }\end{array}$} \\
\hline Teacher explicitly teaches & $\mathrm{x}$ & $\mathrm{x}$ & & & & \\
\hline
\end{tabular}




\begin{tabular}{|l|l|l|l|l|l|l|}
\hline JGT Strategies & Holly & Linda & Melody & Jennifer & Susan & Stephanie \\
\hline $\begin{array}{l}\text { similarities between the home } \\
\text { language and English, such as } \\
\text { cognates, spelling and } \\
\text { comprehension similarities. } \\
\text { (LRT). }\end{array}$ & & & & & & \\
\hline $\begin{array}{l}\text { Teacher ensures that student } \\
\text { knows the meaning of a word } \\
\text { in English before asking the } \\
\text { child to sound it out (LRT). }\end{array}$ & & & & & & \\
\hline $\begin{array}{l}\text { Teaches ensure that students } \\
\text { are able to perceive and } \\
\text { produce distinctive sounds in } \\
\text { English before isolating those } \\
\text { sounds in reading (LRT). }\end{array}$ & & & & & \\
\hline $\begin{array}{l}\text { Teacher encourages child to } \\
\text { read in his or her home } \\
\text { language and English (LRT) }\end{array}$ & & & & & & \\
\hline $\begin{array}{l}\text { Teacher explicitly teaches } \\
\text { American sentence structures } \\
\text { (LRT) }\end{array}$ & & & & & & \\
\hline $\begin{array}{l}\text { Teacher explicitly teaches } \\
\text { organization of a written piece. } \\
\text { (LRT). }\end{array}$ & & & & & \\
\hline $\begin{array}{l}\text { Teacher encourages and allows } \\
\text { for a first draft in the child's } \\
\text { home language. (LRT). }\end{array}$ & & & & & & \\
\hline $\begin{array}{l}\text { With newcomers, teachers } \\
\text { speak slowly, deliberately with } \\
\text { clear vocabulary and diction, } \\
\text { and by pausing more } \\
\text { frequently for longer periods of } \\
\text { time. (LRT) }\end{array}$ & & & & & & \\
\hline $\begin{array}{l}\text { Teachers avoid idiomatic } \\
\text { language or uses it after having } \\
\text { defined it. (LRT) }\end{array}$ & & & & & & \\
\hline $\begin{array}{l}\text { Teachers encourage students to } \\
\text { speak their native languages } \\
\text { with families and friends. } \\
\text { (LRT) }\end{array}$ & & & & & & \\
\hline $\begin{array}{l}\text { Teachers offer opportunities } \\
\text { for students to write for } \\
\text { meaningful purposes. (LRT). }\end{array}$ & & & & & & \\
\hline $\begin{array}{l}\text { Share child's culture with class. } \\
\text { (LRT) }\end{array}$ & $\mathrm{x}$ & & & & & \\
\hline \hline
\end{tabular}




\begin{tabular}{|l|c|c|c|c|c|c|}
\hline JGT Strategies & Holly & Linda & Melody & Jennifer & Susan & Stephanie \\
\hline \hline TOTAL LRT & $\mathbf{6}$ & $\mathbf{6}$ & $\mathbf{3}$ & $\mathbf{3}$ & $\mathbf{4}$ & $\mathbf{7}$ \\
\hline \hline
\end{tabular}

